\title{
German Congress of Laboratory Medicine: 16th Annual Congress of the DGKL (German Society of Clinical Chemistry and Laboratory Medicine)
}

"These abstracts have been reproduced directly from the material supplied by the authors, without editorial alteration by the staff of this Journal. Insufficiencies of preparation, grammar, spelling, style, syntax, and usage are the authors.

Magdeburg, Germany, September 25-28, 2019

Under the auspices of

International Federation of Clinical Chemistry and Laboratory Medicine

European Federation of Clinical Chemistry and Laboratory Medicine

\section{Congress President}

Berend Isermann (Magdeburg)

Sponsor of Abstract awards

Dr. Neumann \& Kindler Ltd. \& Co. KG (Bochum, Germany)

\author{
Scientific Committee
}

\begin{abstract}
Aufenanger Johannes; Achenbach Peter; Balling Rudi; Baum Hansjörg; Bidlingmaier Martin; Biemann Ronald; Bikker Rolf; Blanchard Veronique; Bobrowski Andreas; Borucki Katrin; Burkhardt Ralph; Ceglarek Uta; Dittrich Julia; Fiedler Martin Georg; Findeisen Peter; Fischer Andreas; Frank Helga; Freckmann Guido; Friedrich Alexander; Frye Maike, Fuchs Tina; Geilenkeuser Wolf-Jochen; Gessner André; Günther Claudia; Hameister Erik; Heins Michael; Heyer Robert; Hofmann Walter; Isermann Berend; Kappert Günther; Kappert Kai; Kiehntopf Michael; Klawonn Frank; Kleinridders André; Köck Thomas; Krishnamoorthy Gurumoorthy; Kulikowski Ines; Lackner Karl; Lämmle Bernhard; Lange Peter; Leichtle Alexander; Lianidou Evi; Lichtinghagen Ralf; Lorentz Thomas; Ludwig Jochen; Luppa Peter B.; Mei Henrik; Mohnike Klaus; Möhnle Patrick; Nauck Matthias; Pessler Frank; Peter Andreas; Pick Karl-Heinz; Plötzsch Bernd; Rapp Erdmann; Reis Celso; Reisch Nicole; Renné Thomas; Renz Harald; Ringel Johannes; Rosen John; Rossmann Heidi; Ruland Jürgen; Scheffe Nina; Schimanski Sven; Schlaberg Robert; Schlüter Dirk; Schmid Sebastian; Spannagl Michael; Streichert Thomas; Strowig Till; Tauber Rudolf; Thevis Mario; Thiel Christian; Thiery Joachim; Tolios Alexander, Uhr Manfred; Vogeser Michael; von Ahsen Nicolas; Wagner Robert; Wahida Adam; Wiegel Bernhard; Winter Christof
\end{abstract}




\title{
Analytische Qualität, Labormanagement und Digitalisierung - 1
}

\section{PO-01 / PV-02}

\author{
Using metabolite profiling to unravel potential quality indicators to assess a delayed sample \\ processing in serum
}

\author{
Sven Heiling1; Nadine Knutti; Nicolle Schwarz²; Jörg Geiger³; Juliane Dorow; Uta Ceglarek ${ }^{5}$; Michael \\ Kiehntopf ${ }^{6}$
}

${ }^{1}$ Institut für klinische Chemie und Laboratoriumsdiagnostik, Integrierte Biobank Jena, Jena, Germany ¿University Hospital Jena, Institute of Clinical Chemistry and Laboratory Diagnostics, Jena, Germany ${ }^{3}$ University Hospital Würzburg, Interdisciplinary Biomaterial and Databank Würzburg (IBDW), Würzburg, Germany

${ }^{4}$ University Hospital Leipzig, Institute for Laboratory Medicine, Clinical Chemistry and Molecular Diagnostics, Leipzig, Germany

${ }^{5}$ University Hospital Leipzig, Institute for Laboratory Medicine, Clinical Chemistry and Molecular Diagnostics, Leipzig, Germany

'University Hospital Jena, Institute of Clinical Chemistry and Laboratory Diagnostics and Integrated Biobank Jena, Jena, Germany

Background: Omics advancements are influenced by ineffectively characterized and harmonized preanalytical conditions that can be a noteworthy wellspring of fluctuation. This variation causes in between $50-85 \%$ of clinical errors in clinical diagnostic testing. Consequently, quality indicators must be examined to survey the nature of body liquids and guarantee reliable biomedical research.

\begin{abstract}
Methods: Human serum was subjected to prolonged incubation at room temperature before centrifugation. An extensive metabolite profiling was performed using a high-resolution mass spectrometry-driven approach followed by pathway and chemical similarity enrichment using the webbased tools MetaboAnalyst and ChemRICH. Furthermore, targeted tandem-MS analyses were used to quantify the selected quality indicators in samples of healthy volunteers and patients with cardiologic and rheumatologic diseases.
\end{abstract}

Results: We investigated the metabolome of serum samples from a healthy cohort $(n=10)$ after 0.5 and $2 \mathrm{~h}$ pre-centrifugation delay. To rapidly select quality indicators we performed a chemical similarity enrichment analysis and discovered several highly promising clusters of chemically similar compounds that belong to the eicosanoid metabolism, as well as blood coagulation factors, purine, and pyrimidine derivatives. Additionally, we applied a pathway enrichment approach on the identified clusters and designed scores for promising metabolic ratios. We calculated diagnostic thresholds such as the HIDScore (Hypoxanthine/Inosine/Dihydroorotate-Score), investigated the eicosanoid metabolism and verified our results using additional cohorts of healthy volunteers and patients $(n=50)$ showing a high prediction accuracy with AUROC-values of 0.92 for TTC $<60 \mathrm{~min}$.

Conclusion: The assessment of the quality of body fluids is challenging in clinical research and diagnostics. To guarantee high-quality biospecimen, quality indicators are of utmost importance, and we conceive that this work will stimulate future research into these potential metabolites/metabolic ratios and their possible role as quality biomarkers in biobanking. 


\section{PO-02}

Lactate dehydrogenase activity in serum and heparin plasma samples is affected by filling volume in vacuum tubes

Nick Neuwinger ; Dirk Meyer zum Büschenfelde ${ }^{1}$; Rudolf Tauber ${ }^{1}$; Kai Kappert²

${ }^{1}$ Charité-Universitätsmedizin Berlin, Institut für Laboratoriumsmedizin, Klinische Chemie und Pathobiochemie, Berlin, Germany

²Labor Berlin - Charité Vivantes GmbH, Laboratoriumsmedizin und Toxikologie, Berlin, Germany

BACKGROUND: The activity of lactate dehydrogenase (LDH) is routinely monitored, e.g. for stratification of malignant diseases, but is susceptible to preanalytical influences.

METHODS: Blood was withdrawn by antecubital venipuncture from healthy volunteers and collected in vacuum blood collection tubes (serum, SE; heparin plasma, HP). The tubes were either filled completely ( $\geq 90 \%$ of volume), or were underfilled to approximately $50 \%$. Thereafter, tubes were centrifuged directly or stored at room temperature for $4 \mathrm{~h}$ prior to centrifugation. We systematically analyzed potassium, sodium, chloride, LDH, creatine kinase, total cholesterol, and indices for hemolysis, icterus, and lipemia. In a subset of tubes we also measured blood filling velocity. Finally, in an in-patient cohort of more than 74,000 patients, we analyzed plasma yield and data distribution of LDH by means of the DGKL Reference Limit Estimator (RLE) to reconfirm our findings in healthy volunteers.

RESULTS: The activity of LDH was higher in HP tubes compared to SE. The reduction of the filling volume of tubes was followed by significantly higher LDH values (SE: +21.6\%; HP: +28.3\%), potassium (SE: $+4.2 \%$; HP: $+5.3 \%$ ), and hemolysis index (SE: $+260.8 \%$; HP: $+210.0 \%$ ). In contrast, levels of other analytes remained generally stable. The filling velocity of blood in the collection tubes was approx. 3-fold higher in the first compared to the second half with no differences between HP and SE. Notably, the plasma yield in the routine patients cohort also inversely correlated with the activity of LDH. Estimation of reference limits for LDH revealed established intervals only in patients' tubes that were adequately filled.

CONCLUSIONS: The reduction of filling volume in both SE and HP collection tubes leads to significant increases in LDH activity, both in healthy volunteers and in inpatients. This may be considered in cases of clinically implausible enhanced levels of LDH. 
PO-03 Experiences from 6 Years of Quality Assured Model of End-Stage Liver Disease (MELD)
Diagnostics

Pascal Hunold ${ }^{1}$; Daniel Seehofer ${ }^{2}$; Thomas Berg ${ }^{3}$; Joachim Thiery ${ }^{1}$; Thorsten Kaiser ${ }^{1}$

'University Hospital Leipzig, Institute of Laboratory Medicine, Clinical Chemistry and Molecular Diagnostics, Leipzig, Germany

'University Hospital Leipzig, Department of Visceral, Transplant, Thoracic and Vascular, Surgery, Leipzig, Germany

${ }^{3}$ University Hospital Leipzig, Section of Hepatology, Clinic and Polyclinic for Gastroenterology, Leipzig, Germany

Introduction

The MELD (Model of End- Stage Liver disease) score is established for allocation of liver transplants and has to guarantee a sensitive, specific, objective and fair organ allocation. The score is based on three medical laboratory parameters: bilirubin, creatinine and international normalized ratio (INR).

Methods

In 2012 the University Hospital Leipzig created and established a quality ensured lab-MELDDiagnostic. Every MELD score is directly requested at the laboratory system, calculated and specifically validated by laboratory physicians. Within this process the three parameters as well as the MELD score are checked for plausibility in the context of pre-values and other patients results. This study analyses the experiences since implementation of the laboratory medical quality assurance system for MELD-Diagnostics.

Results

Since 2012, 7270 MELD scores were requested. The majority of these requests could be reported to Eurotransplant without any hint of limited validity. However, 202 scores could not be reported initially, 145 of these cases could not have been reported to Eurotransplant at all for various reasons. In 34 cases the dialysis status did not meet the requirements and 1 case of unstated intake of oral anticoagulation could be determined and corrected within the process of validation.

\section{Discussion}

The laboratory medical quality assurance system for MELD-Diagnostics was effective in identification of results with limited validity. Therefore, it should be applied to transplant centers to prevent diagnostic errors with possible adverse effects for the patie. 


\title{
PO-04
}

\section{Reduction of hemolysis rate by using VACUETTE® HOLDEX® Single-Use Holder or VACUETTE® SAFELINK blood collection device}

\author{
Sirid Griebenow'; Giuseppe Lippi; Gian Luca Salvagno² \\ ${ }^{1}$ Greiner Bio-One GmbH, Produkt Management, Kremsmünster, Austria \\ ${ }^{2}$ University of Verona, Laboratory of Clinical Chemistry and Hematology, Verona, Italy
}

Background: Hemolysis is one of the main sources for sample rejection in a clinical laboratory as it can strongly influence the reliability of results. Using an intravenous line for blood collection frequently causes hemolysis. The target of two studies was to show the performance of the HOLDEX® single use holder and SAFELINK in reducing the hemolysis rate by preventing erythrocyte ruptures, whereby these holders have potential to reduce shear stress caused by differences of pressure between veins and evacuated tube systems. A flash chamber which reduces the flow rate results in a softer draw.

Methods: Blood was drawn from 60 ED patients in Italy into VACUTAINER SST II Plus tubes with a $20 \mathrm{G}$ catheter and using a conventional holder (VACUTAINER One Use Holder, Becton Dickinson) or VACUETTE $®$ HOLDEX (Greiner Bio-One). All tubes were centrifuged according to the manufacturers' instructions. Serum was tested for potassium, lactate dehydrogenase (LDH) and hemolysis index (HI). In an additional second study, 30 healthy volunteers' blood were drawn to determine 24 chemistry and immunochemistry analytes in lithium-heparin plasma (on Cobas 8000 , Roche), hematology parameters in EDTA plasma (on Sysmex XN, Sysmex, Japan) and hemostasis parameters in citrate plasma (on ACL Top 700, Instrumentation Laboratory, USA) using either HOLDEX or SAFELINK in paired specimens. Blood collection was based on two sequential venipunctures, on one arm by using a 19 gauge straight luer needle attached to a HOLDEX and on the other arm using a 19 gauge straight needle attached to SAFELINK. All subjects provided informed consent and approval by ethical committee was given.

Results: In samples collected with VACUTAINER One Use Holder, concentrations of potassium (4.25 vs. $4.16 \mathrm{mmol} / \mathrm{L}), \mathrm{LDH}$ (498 vs $459 \mathrm{U} / \mathrm{L}$ ) and cell-free hemoglobin (fHb) (0.42 vs. $0.22 \mathrm{~g} / \mathrm{L})$ were higher than with HOLDEX. The frequency of samples with $\mathrm{fHb}>0.5 \mathrm{~g} / \mathrm{L}$ was not statistically significant, whilst that of grossly hemolyzed samples was significantly higher for samples collected with VACUTAINER One Use holder. The additional comparison study of HOLDEX and SAFELINK did not result in significant differences. Potassium was slightly higher ( $4.38 \mathrm{vs.} 4.49 \mathrm{mml} / \mathrm{L}$ ), but the percent bias remained within quality specifications. Of 30 samples, the rate of samples with $\mathrm{HI}>3$ was lower when blood was collected with SAFELINK than with HOLDEX.

Conclusion: The results confirm the reduction in hemolysis rate when drawing blood from an intravenous line and using the holder HOLDEX or SAFELINK, as the specimen quality for parameter analysis is comparable when blood is collected by either of them. Both holders have potential to decelerate the blood flow pressure from veins into an evacuated blood collection tube, thus increasing the effectivity in the laboratory work flow by reducing the number of specimens requested repeatedly due to hemolysis. 


\title{
PO-05
}

\section{Combination of informative biomarkers in small pilot studies and estimation of the sample size for extended studies}

\author{
Amani Al-Mekhlafi'; Frank Klawonn ${ }^{2}$ \\ ${ }^{1} \mathrm{Helmholz}$ Centre for Infection Research, Microbial Immune Regulation, Biostatistics, Braunschweig, \\ Germany \\ ${ }^{2}$ Ostfalia Hochschule für angewandte Wissenschaften / Helmholtz-Zentrum für Infektionsforschung, \\ Biostatistics, Braunschweig, Germany
}

Recently, Biomarkers are used widely in medical researches to detect the disease early which nowadays plays an important role in modern clinical and preventive medicine. According to the published data by GVK BIO Online Biomarker [1], over one hundred thousand biomarkers have been found, and abundance of biomarkers are discovered every year. As consequence, so many biomarkers can be studied when there is a new challenge. To avoid the waste of money and time, it is suggested to control the number of patients strictly at the beginning. For this reason, the pilot studies are commonly did in such studies as a necessary first step. The high AUC abundance (HAUCA) curves is a method that can show the high correlation of biomarkers with the disease exceed the pure random effect. By comparing the number of biomarkers that exceed specific value of AUC in the real data to the random data we can know if the data is worthy to be extended with bigger sample size. Expanding pilot studies sample size allows the researchers to determine at which size the selected markers are highly likely to have performance that is at or above the target level and the probability of finding the difference between the groups just by chance is controlled. However due to the small number of observation and high number of biomarkers, we expect high AUC values just by chance. By Wilcoxon-Mann-Whitney-U test, we compute the probability that an attribute with $\mathrm{n}$ values randomly assigned to two classes with a given prevalence exceeds a given AUC value [2]. To remain the probability of getting at least one significant result just due to chance below the certain significant level, the multiple testing procedures are used (Bonferroni correction)[3]. The new sample size has been estimated by choosing a specific value of AUC to be validated and we assume the prevalence will be the same in the extended study as in the pilot. We increase $n$ (No. of observations) and $n+(\mathrm{No}$. of positive class) gradually until we get the same AUC value with a significant corrected $p$-value. In the medical research, even if the performance of the feature is very high, is still not sufficient to provide sufficient accuracy [4,5]. For that, the researchers hope to achieve higher performance value and better efficiency of the statistical analysis by the improvement in the performance measure that obtained from the combination of biomarkers. Different modeling strategies have been proposed to select and combine biomarkers. However the ROC curve has turned out to be a standard tool for evaluating the diagnostic accuracy of a classifier [6,7]. In our study, we evaluate features by calculating the AUC of each single feature and the k top-ranked features with maximum AUC are selected. Furthermore, we address an important approach that are not widely explored in the literatures which is the correlation of the biomarkers within the groups. Bootstrapping has been used to measure the confidence interval for this combination. 


\section{PO-06}

Reliable Minimal Difference of Glucose concentration measurements based on ten year data

Sandra Keutmann'; Anders Kallner²; Anke Hannemann"; Nele Friedrich'; Stephanie Zylla'; Mathilde Dahl'; Matthias Nauck'; Astrid Petersmann ${ }^{1}$

${ }^{1}$ Universitätsmedizin Greifswald, Institut für Klinische Chemie und Laboratoriumsmedizin, Greifswald, Germany

${ }^{2}$ Karolinska University Hospital, Department of Clinical Chemistry, Stockholm, Sweden

Introduction:

Diagnosis of diabetes mellitus is based on cut-off values e.g. plasma glucose concentrations. Even though a measurement result is only complete when the attached measurement uncertainty is also reported, imprecision in laboratory measurements is rarely considered in patient care. Lately, the German Diabetes Society guideline on definition, classification and diagnosis on diabetes mellitus recommended to measure plasma glucose with an imprecision given as a minimal difference of 0.7 $\mathrm{mmol} / \mathrm{L}$, or less, at a concentration of $7.0 \mathrm{mmol} / \mathrm{L}$. The MDcut-off describes the smallest analytical difference between a measurement and a cut-off which would be regarded as statistically significant different at a $95 \%$ level of confidence, if a coverage factor $(k)$ of 2 is used.

Material and Methods:

Two approaches were included to obtain imprecision: 1. Long-term imprecision based on Rili-BAEK based $24 / 7$ patient care internal quality control (IQC) data (four concentrations) over a period of ten years and 2 . detailed short-term imprecision assessed by hourly measurements of control materials (three concentrations) on three measuring systems connected to a laboratory automation used for glucose concentration measurement in the investigating laboratory. Minimal Difference for evaluation of a measurement result in relation to a cut-off value was $\llbracket M D \rrbracket$ (cut-off) $=k \times S D=2 \times S D(k=2)$. To obtain representative values suitable for use in the diagnosis of diabetes mellitus the $95 \%$ percentiles of the MDcut-off distributions for each concentration were calculated and used to derive a linear equation that allowed calculating MDcut-off for further glucose concentrations.

Results \& Discussion:

Based on ten years of IQC data MDcut-off at the diagnostic cut-off of $7.0 \mathrm{mmol} / \mathrm{L}$ was found to be 0.45 $\mathrm{mmol} / \mathrm{L}$. In the short-term experiment MDcut-off at $7.0 \mathrm{mmol} / \mathrm{L}$ was $0.38 \mathrm{mmol} / \mathrm{L}$. MDcut-off remained below the recommended limit of $0.7 \mathrm{mmol} / \mathrm{L}$ in both experiments. The variability introduced by slight performance differences within and between instruments can be covered by reporting the long term MDcut-off across all connected instruments. This procedure is recommended when multiple analysers are used in parallel, because the measurement uncertainty should be calculated for the complete laboratory and expressed per analyte as one MDcut-off value.

\section{Conclusion}

In this study setting stable results for MDcut-off were obtained after about thirty independent control cycles of MDcut-off. In the study setting this number was reached after one year, because the data from three analysers were combined. Medical laboratories may use IQC of an appropriate time interval to provide a reliable MDcut-off along with glucose concentration results used for diagnosis of diabetes mellitus. 
PO-07

\title{
Patient blood management: Single tube technology
}

\author{
Astrid Petersmann ${ }^{1}$; Matthias Nauck ${ }^{1}$
}

${ }^{1}$ Universitätsmedizin Greifswald, Institut für Klinische Chemie und Laboratoriumsmedizin, Greifswald, Germany

Introduction:

Patient blood management has become an important focus in providing laboratory results in patient care (1). The intention is to only conduct as few blood collections with as little blood volume as possible to avoid unnecessary blood loss for the patient. So far different tubes are needed for clinical chemistry, hematology and coagulation tests, each with a minimum volume and a dead volume. A new blood collection tube STTMP (Single tube technology multi parameters; Kabe, Nümbrecht, Germany) enables laboratories to conduct all tests in only one tube reducing the blood volume per draw by approximately $60 \%$ ( $4.5 \mathrm{ml}$ Plasma, $2.7 \mathrm{ml}$ Citrate Plasma, $2 \mathrm{ml}$ EDTA whole blood vs. $3.5 \mathrm{ml}$ ). The German Rili-BAEK, as a document representing legal requirements, "aims to ensure, in particular, that Influencing factors and in-vitro effects during the pre-analytical phase are minimized [...]" (2). Therefore the study evaluates whether the test results obtained from blood samples collected in STTMP are comparable to results collected in other currently commercially available tubes.

Methods:

Blood from ten healthy volunteers was obtain by venous puncture. About 50 basic clinical chemistry, hematology and coagulation analytes were measured in primary tubes from Sarstedt and Becton Dickinson and compared to the new collection tube. Ethical approval and informed consent were provided prior to sampling. Analysers used in this study were: Dimension Vista 1500 for Clinical Chemistry and the Sysmex CS 2500 for coagulation (both Siemens Healthcare, Eschborn, Germany), TOSOH G8 for HbA1c (TOSOH Bioscience Europe, Griesheim, Germany), and Sysmex XN for hematology (Sysmex, Norderstedt, Germany).

Results \& Discussion:

Significant differences of results between the established manufacturers Sarstedt and BD occurred in $10 \%$ of the analytes. About $50 \%$ of all investigated analytes did not differ significantly from either of the established primary tubes. For use in clinical settings a set of analytes could be identified that can be measured using the investigated new tube. Further analytes could be included in this set, if reference intervals were determined specifically for this material.

\section{Conclusion}

The new blood collection tube represents a promising approach to promote patient blood management and lean processes in modern medical laboratories and for the clinical partners. Further development will be needed to include more analytes.

1. Meybohm P, Richards T, Isbister J, Hofmann A, Shander A, Goodnough LT, et al. Patient Blood Management Bundles to Facilitate Implementation. Transfusion medicine reviews. 2017;31(1):62-71.

2. Revision of the "Guideline of the German Medical Association on Quality Assurance in Medical Laboratory Examinations - Rili-BAEK" (unauthorized translation). J Lab Med 2015;39(1):26-69 


\title{
PO-08
}

\section{0-year rejection rates due to hemolysis from a maxium care hospital}

\author{
Lars Linnemann'; Mathilde Dahl'; Matthias Nauck'; Astrid Petersmann \\ 'Universitätsmedizin Greifswald, Institut für Klinische Chemie und Laboratoriumsmedizin, Greifswald, \\ Germany
}

Introduction:

Hemolysis is a very common problem associated with drawing and processing of diagnostic blood samples and may occur in all parts of the pre-analytical phase, i.e. handling, interim storage and transport of samples. Hemolysis monitoring is often conducted only intermittently or for short period of times up to a few years or restricted to parts of a given hospital, e.g. emergency room. In this study we monitored hemolysis rates in a maximum care hospital over a period of ten years.

Material and Methods:

Hemolysis is noticed by the clinician when samples are rejected. Among important clinical analytes sensitive to hemolysis are potassium and INR. Here we evaluated hemolysis based on sample rejections for those two analytes. Rejection of samples with orders for potassium was based on HIL index determined by the Dimension Vista (Siemens Healthcare GmbH, Eschborn, Germany). Rejection of samples with orders for INR assessment in citrate samples was based on visual inspection until 2014 for the BCS and on HIL index measured by the CS (both also Siemens Healthcare $\mathrm{GmbH}$ ). Data on rejection rates (number of analysed and rejected orders) due to hemolysis was retrieved from the laboratory information system for the years $2007-2016$. We report yearly $95 \%$ percentiles and medians of hemolysis rates: overall, for different ward types (intensive care, normal, ambulatory, and emergency) and frequency of blood draws, respectively.

\section{Results}

Over two million measurements were included in the study: 1156,119 for potassium and 921,464 for INR. Of 150 wards 42 had data for the whole study period accounting for 1,909,996 (92\%) of the measurements. Median rejection rates due to hemolysis continuously increased over the study period. At the end of the study period median (95\% percentile) rejection rates for potassium and INR were on average $1 \%(3.6 \%)$ and $1.9 \%(7.1 \%)$, respectively. There was no relationship between average number of blood draws and rejection rates with one exception: median INR was lower on wards with less than one blood draw per day. Also differences between ward types were observed with highest rejection rates in emergency rooms and lowest in intensive care units. The introduction of a new pneumatic tube system did not coincide with increased rejection rates whereas the introduction of a new coagulation instrument did.

\section{Discussion and Conclusion:}

Hemolysis remains an important pre-analytical issue. Emergency rooms are at high risk for rejection of samples due to hemolysis. The observed overall increase of rejection rates is accompanied with increasing numbers of samples, thus indicating that the workload for blood drawing staff increased during the study period with possible implication for hemolysis rates. Also stricter pre-analytical requirements for instruments may lead to higher rejection rates. Our data also demonstrate that the introduction of a new pneumatic tube system did not lead to an increase in hemolysis based rejections rate. 


\title{
Analytische Qualität, Labormanagement und Digitalisierung - 2
}

\author{
PO-09 / FV-06
}

\section{A Digital Approach to Improve the Management of Patients with Inflammatory Immune Response (AMPEL)}

Sebastian Wegener'; Felix Eckelt'; Martin Federbusch'; Carina Cundius²; Markus Voigt'; Alexander $K_{\text {Kehrer }}^{3}$; Jörg Telle ${ }^{3}$; Thorsten Kaiser ${ }^{1}$

'Universitätsklinikum Leipzig Inst. f. Laboratoriumsmedizin, Klinische Chemie und Molekulare Diagnostik, AMPEL, Leipzig, Germany

${ }^{2}$ Universitätsklinikum Leipzig, Bereich 1 - Informationsmanagement, Leipzig, Germany

${ }^{3}$ Xantas AG, AMPEL, Leipzig, Germany

Introduction

Procalcitonin (PCT) is an important biomarker for the diagnosis and treatment of septic patients. The interpretation of the PCT results over time as well as obtaining the adequate medical consequences is challenging.

A clinical decision support system (CDSS) could improve timely interpretation and reaction and therefore the post-analytical quality. At the University Hospital of Leipzig, a project of digital laboratory medicine (AMPEL), that includes PCT diagnostics, has been introduced. The aim of our study is to design a feasible and effective CDSS also for complex diagnostics that proves its benefit for clinical care.

\section{Methods}

A retrospective analysis of all patient results from the laboratory and clinical information system in 2018 at the University Hospital of Leipzig was performed to design the framework of the CDSS. The resulting system is currently under prospective evaluation. The clinically relevant cut-off for PCT was defined as $1.0 \mathrm{ng} / \mathrm{mL}$ according to the literature and clinical guidelines. Frequency and time difference of follow-up measurements were systematically investigated to analyzed adherence to sepsis guidelines.

\section{Results}

We observed 23425 PCT results in sera of 4842 patients ( 2111 female, 2731 male). The median concentration was $0.40 \mathrm{ng} / \mathrm{mL}$ (male $=0.46 \mathrm{ng} / \mathrm{mL}$, female $=0.32 \mathrm{ng} / \mathrm{mL}, \mathrm{p}=0.001$ ). In 1465 patients (30.3\%, 7999 specimens) the PCT results were $>1.0 \mathrm{ng} / \mathrm{mL}$.

We further analyzed the time between the measurement and follow-up measurement. The median time was $20 \mathrm{~h}$. A PCT result $>1 \mathrm{ng} / \mathrm{mL}$ was re-examined within 48 hours in 363 of 1465 patients (148 female, 215 male, in total 6513 specimens). Accordingly, 1102 (75.2\%) patients received no follow-up within $48 \mathrm{~h}$.

\section{Conclusion}

PCT is frequently used as a biomarker for patients with systemic inflammatory immune response and bacterial infections. Guidelines recommend reinvestigations of PCT in at least $<48$ hours for patients with significantly elevated initial results. Our preliminary data indicate limited adherence to the recommendations. However due to the complexity of the diagnostics (e.g. clinical situation of the patient, course of other biomarkers such as CRP, IL-6) an adequate management cannot depend on one biomarker solely. Thereby, our CDSS, which is currently in development, will combine the laboratory diagnostics and further clinical data. We are convinced that AMPEL could improve the management of patients with inflammatory immune response. 


\title{
PO-10 / PV-01
}

\section{Biotin interference in high-sensitivity in-vitro diagnostics - Analysis of field safety notices published by the BfArM in Germany}

\author{
Jürgen Hannigi'; Tanja Grammer²; Rüdiger Siekmeier'; Winfried März ${ }^{3}$ \\ ${ }^{1}$ Pharmazeutisches Institut der Universität Bonn, Drug Regulatory Affairs, Bonn, Germany \\ ${ }^{2}$ Medizinische Fakultät Mannheim der Universität Heidelberg, Medical Clinic V, Mannheim, Germany \\ ${ }^{3}$ Synlab Holding Deutschland GmbH, Synlab Academy, Mannheim, Germany
}

Since 20 years the European Directive 98/79/EC on In-vitro Diagnostics (IVD) regulates marketing/post market surveillance of IVD in the European Economic Area. In cases of incidents and field safety corrective actions (FSCA) manufacturers have to inform responsible Competent Authority (CA) and public by field safety notices (FSN). In Germany FSCA/FSN are published on the BfArM homepage (CA for most IVD). Streptavidin-biotin-based tests serve for detection of low concentrated analytes. Biotin often administered in high dose for treatment (e. g. $600 \mathrm{mg} / \mathrm{day}$ in multiple sclerosis, recommended dose $30 \mu \mathrm{g} /$ day) or nutrition supplement interferes with the detection method. Since 2017 there were several publications (e. g. JAMA 2017;318(12):1150-1160) and safety communications from health agencies (e. g. FDA 2017: https://www.fda.gov/medicaldevices/safety/alertsandnotices/ucm586505.htm, BfArM 2019: https://www.bfarm.de/SharedDocs/Risikoinformationen/Pharmakovigilanz/EN/RHB/2019/rhbbiotin.html) on this topic. We evaluated FSCA/FSN for IVD regarding biotin interference published by BfArM (http://www.bfarm.de/DE/Medizinprodukte/riskinfo/kundeninfo/functions/kundeninfo-node.html).

Analysis was made for FSCA/FSN published between end 2004 (begin of publication) and end of June 2019. Interferences other than biotin, e. g. pharmaceuticals were excluded.

Until June 2019 about 8950 (about 8500 since begin 2005; data 2018/19 estimated as not yet published) notifications for IVD were received by BfArM, resulting in 2743 FSCA since December 2004. 13 FSCA were found for biotin interference including follow-up FSN, the first in 2013, others 2016 (1), 2017 (4), 2018 (5) and 2019 (2). The number of tests is higher as most manufacturers include several tests/platforms in one FSCA. Biotin interference affects 51 tests, e. g. hormones (aldosterone, TSH, T3, T4, fT3, fT4), proteins/proteohormones (prolactin, FSH, chromogranin, thyreoglobulin, thyreoglobulin-autoantibody, CK-MB mass, free PSA, Tnl, HBc lgM, allergen specific IgG4) and drugs (DHEA-SO4, folate, vitamin D, infliximab, adalimumab, sirolimus, cyclosporine). FSN described type (decreased results in sandwich tests (29), increased results in competitive tests (15)) and in part intensity of interference dependent on biotin concentration, underlying cause (biotin interference (13)), (potential) consequences for patients (7; no report of occured patient harm), requirement for retesting (recommended/not necessary; 1/9), provided dose and/or plasma/serum concentration without interference (13) and often announced an update of the instructions for use (10).

The number of FSN reporting biotin interference is small. As biotin is often administered without knowledge of health care providers (e. g. supplements) and some tests are of high relevance, manufacturers/physicians should be aware of the interference. Although most FSN fulfil MEDDEV criteria the quality should be further improved as they play a central role in FSCA. 


\section{PO-11}

\section{Introduction of a Laboratory Based Decision Support System to Increase Patient Safety (AMPEL)}

Felix Eckelt'; Martin Federbusch'; Johannes Remmler"; Carina Cundius²; Markus Voigt'; Alexander Kehrer $^{3}$; Jörg Telle; ${ }^{3}$ Thorsten Kaiser ${ }^{1}$

${ }^{1}$ Universitätsklinikum Leipzig Inst. f. Laboratoriumsmedizin, Klinische Chemie und Molekulare Diagnostik, AMPEL, Leipzig, Germany

'Universitätsklinikum Leipzig, Bereich 1 - Informationsmanagement, Leipzig, Germany

${ }^{3}$ Xantas AG, AMPEL, Leipzig, Germany

Introduction

There is an increasing number and complexity of medical diagnostics. Physicians are aware of missed laboratory results and as a consequence adverse events for patients. Clinical decision support systems (CDSS) may improve timely interpretation and adequate reaction. At the University Hospital of Leipzig, a project of digital laboratory medicine (AMPEL) was recently introduced. The aim of our project is to establish a CDSS adapted to the requirements of the clinician to prove necessity, feasibility and effectiveness in the clinical context. The treatment of severe hypokalemia is used as a first prove of concept.

Methods

The potassium measurements in serum, plasma and whole blood from the Institute of Laboratory Medicine (ILM) and the Point of Care (POCT) devices at the University Hospital of Leipzig from 2018 were systematically analyzed for severe hypokalemia $(<2.5 \mathrm{mmol} / \mathrm{L})$. In these patients we analyze the time lag between potassium measurements and the proportion of cases where a control measurement was not carried out after at least $6 \mathrm{~h}$. The findings were used to design a feasible CDSS framework for implementation in the healthcare information system.

Results

We retrospectively investigated 417,789 potassium results from 37,900 patients. Hypokalemia was diagnosed more often in sera (22,242 patients) than in whole blood (15,658 patients). Severe hypokalemia was identified in 443 specimens of 317 patients and was less frequently in sera $(n=117)$ compared to POCT-measurements $(n=326)$. In $100(23 \%)$ cases the critical hypokalemia was not reexamined within 6 hours. Of these cases 50 were from non-internal wards, 20 from internal wards and 30 from intensive care units and emergency rooms. The delay in follow-up diagnostics was $21.5 \mathrm{~h}$ in median for non-internal $23 \mathrm{~h}$ for internal wards and $11 \mathrm{~h}$ on intensive care units $(p<0.05)$.

\section{Conclusions}

Severe hypokalemia is a frequent and critical medical finding. Due to the serious complications up to ventricular fibrillation a short-term therapy and follow-up measurement is indicated. Our preliminary results show that there is a significant frequency of delayed follow-up measurements. By introduction of our AMPEL-CDSS we are able to detect these patients in real-time. This allows to inform the physicians promptly. The implementation of a digital reporting system in the hospital information system has started with promising first results. The system will be continuously developed with hypokalemia as a first use case and extend to other clinical situations. We are convinced that the AMPEL system has the potential to improve the safety and quality of patient care 
PO-12

\title{
Development of a Laboratory Based Clinical Decision Support System (AMPEL) for the Management of Severe Hyponatremia
}

Tom Sicker'; Martin Federbusch'; Felix Eckelt'; Carina Cundius²; Markus Voigt'; Alexander Kehrer; Jörg Telle; ; Thorsten Kaiser ${ }^{1}$

\author{
${ }^{1}$ Universitätsklinikum Leipzig, Institut für Laboratoriumsmedizin, Klinische Chemie und Molekulare \\ Diagnostik (ILM), Leipzig, Germany \\ ${ }^{2}$ Universitätsklinikum Leipzig, Bereich 1 - Informationsmanagement, Leipzig, Germany \\ ${ }^{3}$ Xantas AG, AMPEL, Leipzig, Germany
}

Introduction

Hyponatremia is a frequent and relevant clinical condition. In severe hyponatremia clinicians need to monitor the blood sodium concentration frequently to prevent serious neurological complications. At the University Hospital of Leipzig, a project of digital laboratory medicine (AMPEL) has been introduced. Severe hyponatremia serves as a sub-project. The aim of our study is to develop a CDSS to provide clinicians with effective support in diagnosing and treatment of patients according to the guidelines.

Methods

A retrospective analysis of all patient results from the laboratory and clinical information system including point-of-care measurements (POCT) in 2018 at the University Hospital of Leipzig was performed. These data in synopsis with recommendations from clinical guidelines were used to design the framework of the CDSS. The resulting system is currently prospectively evaluated. The cut-off for severe hyponatremia was defined as $125 \mathrm{mmol} / \mathrm{L}$. The frequency and time difference of follow-up measurements were analyzed in the context of clinical practice guidelines.

\section{Results}

We observed 414658 sodium results of 56,099 patients (male $=28254$, female $=27843$ ) in 2018 (serum, plasma and whole blood; 221,371 POCT measurements and 193,287 measurements in the Institute of Laboratory Medicine (ILM). The median concentration was $139.3 \mathrm{mmol} / \mathrm{L}$ (male $=139.2$ $\mathrm{mmol} / \mathrm{L}$, female $=139.4 \mathrm{mmol} / \mathrm{L})$. Severe hyponatremia $<125 \mathrm{mmol} / \mathrm{L}$ was identified in $3393 \mathrm{samples}$ $(0.82 \%)$ and 843 patients $(1.5 \%$; male $=463(1.6 \%)$, female $=380(1.4 \%), p=0.008)$. It was observed more often in POCT measurements $(n=2293)$ compared to ILM measurements $(n=1100)$.

Further, the time difference between a result $<125$ and the follow-up result was analyzed: The median was 4.2 h. 2074 severe hyponatremias (61.1\%) have been controlled within $6 \mathrm{~h}$ and $2792(82.3 \%)$ have been controlled within $24 \mathrm{~h}$.

\section{Conclusions}

Our findings indicate limited guideline adherence regarding monitoring and re-examination intervals of patients with severe hyponatremia. The reasons remain speculative und have to be elucidated. On the basis of our findings and further retrospective analysis as well as the available literature and guidelines, we are establishing a CDSS which will provide the physicians with specific information and recommendations. We are convinced that this AMPEL-CDSS could improve the management of severe hyponatremia. 


\section{PO-13}

\section{Heparin anticoagulation as a preanalytical influencing factor on the assessment of blood cell- derived biomarkers}

Rene Huber'; Daniela Küper'; Rozan AttililAbedalkhader'; Bernadette Lüns'; Korbinian Brand'; Karin Weissenborn'; Ralf Lichtinghagen ${ }^{1}$

${ }^{1}$ Medizinische Hochschule Hannover, Institut für Klinische Chemie, Hannover, Germany ${ }^{2}$ Medizinische Hochschule Hannover, Klinik für Neurologie, Hannover, Germany

\section{Background:}

During blood sampling anticoagulants alter biomarker expression differentially and thus may represent an influencing factor in the preanalytical phase for the validity of distinct measurants. We aimed to evaluate the effects of different anticoagulants on biomarker regulation using matrix metalloproteinase (MMP)-9 as an example for cytokine-regulated gene expression in blood cells.

Materials and Methods:

MMP-9 and cytokine expression was determined (ELISA, qPCR, Proteome Profiler Array) in dose/time-dependent experiments. Anticoagulants (HMWH, LMWH, EDTA, citrate) and known contaminants (over sulfated chondroitin sulfate (OSCS)) were applied in individual, double, and triple co-culture experiments using monocytic THP-1, Jurkat T-, and HT B-cells ( $n=3$ each).

Results:

Anticoagulant incubation has no direct influence on MMP-9 expression of monocytic, T-, or B-cell lines. Treatment with HMWH up to $24 \mathrm{~h}$ significantly increased MMP-9 expression in co-culture experiments of all three cell lines (mRNA: 7.1 -fold, $p \leq 0.01$; protein: 3.3 -fold, $p \leq 0.05$ ) or monocytes/Tcells only (mRNA: 7.9-fold, $p \leq 0.05$; protein: 3.3 -fold, $p \leq 0.05$ ) whereas LMWH, citrate, and EDTA did not. Supernatant of T-cells incubated with HMWH also significantly induced MMP-9 in monocytes (mRNA: 8.8-fold, $p \leq 0.05$ ). T-cell cytokine profiling revealed that IL 16/Serpin E1 are specifically secreted in response to HMWH. Monocytes stimulated with IL 16/Serpin E1 showed elevated IL-8 expression (mRNA: 5.6-fold, $p \leq 0.05$ ). T-cell derived IL-16, Serpin E1, and (autocrine) IL 8 induced monocytic MMP-9 expression (mRNA: 7.0-fold, p $\leq 0.05$ ). In addition, the known HMWH contaminant OSCS massively increased MMP-9 expression by monocytes co-cultured with T-cells (mRNA: approx. 700 -fold, $p \leq 0.05$ ).

\section{Conclusion:}

Our results indicate that monocytes show increased production of biomarkers in response to T-cellderived soluble mediators, which are secreted in the presence of $\mathrm{HMWH}$ and known contaminants during blood sampling. 


\title{
PO-14
}

\section{Investigation of the in vitro influence of coagulation factors on human dermal fibroblasts}

\author{
Monika Wolny'; Franziska Knüttgen'; Katharina von Bargen'; Tobias Flieder'; Anne-Kathrin Vollmer'; \\ Cornelius Knabbe'; Ingvild Birschmann ${ }^{1}$
}

${ }^{1}$ Herz- und Diabeteszentrum Nordrhein-Westfalen, Universitätsklinik der Ruhr-Universität Bochum, Institut für Laboratoriums- und Transfusionsmedizin, Bad Oeynhausen, Germany

\section{Background:}

Maintaining integrity is essential for a biological organism. This is ensured by the complex mechanism of wound healing. If the natural course of wound healing is disturbed, the healing process may be delayed, or a chronic wound site may develop. In addition to conventional therapy, new strategies are being developed to treat, for instance, diabetic foot ulcers. A promising method is the application of platelets, the key players of hemostasis, in form of platelet-rich plasma. Furthermore, some coagulation factors have been associated with wound healing, e.g. FIXa could be associated in vitro with reduced cell adhesion and increased migration. In a mouse model it was shown that FXIII is involved in the healing of myocardial infarction. This indicates an important role played by coagulation factors in wound healing besides hemostasis.

\section{Objective:}

The aim of this study was to investigate the influence of a specific coagulation factor deficiency on dermal fibroblasts in vitro.

\section{Methods:}

Cell culture supernatant was supplemented with coagulation-factor-deficient plasma as a protein source. Two concentrations of plasma $(20 \%$ and $60 \%)$ were used. Cell viability, proliferation and intracellular oxidative stress of dermal fibroblasts were evaluated in the absence of a specific coagulation factor. Furthermore, the gene expression of collagen I and IV, matrix metalloproteinase (MMP)2 and MMP9, tissue inhibitor of metalloproteinase (TIMP)1 and TIMP3, as well as the enzyme activity of MMP2 and MMP9, were investigated.

Results:

Cell proliferation, intracellular oxidative stress and enzyme activity of MMP2 and MMP9 did not show any significant changes dependent on specific coagulation factor deficiency. However, in first experiments gene expression of collagen I and IV, MMP2, MMP9 and TIMP1 was increased in cells incubated with plasma lacking FVII, FVIII, FIX or FX. Furthermore, cells showed a significantly increased viability when incubated with clotted supernatants (20\% plasma lacking FVII, FVIII, FIX, FX, FXI, FXII or FXIII).

\section{Conclusion:}

Contrary to references in the literature, no significant effect of individual coagulation factors could be observed. However, the analyses indicate an influence of clot formation in the cell culture supernatant. 
PO-15

Interference of high dose biotin supplementation with time-critical immunoassays utilizing the interaction between streptavidin and biotin: evaluation of a countermeasure

Ingo Mrosewski'; Matthias Urbank'; Thomas Stauch²; Rafael Switkowski ${ }^{1}$

${ }^{1} \mathrm{MDI}$ Limbach Berlin GmbH, Laboratoriumsmedizin, Berlin, Germany ${ }^{2}$ MVZ Labor PD Dr. Volkmann und Kollegen, Klinische Chemie/Toxikologie, Karlsruhe, Germany

Aim: Automated immunoassays utilizing the interaction between streptavidin and biotin are commonly used for a wide variety of time-critical and standard clinical chemical parameters. High dose biotin supplementation can lead to clinically significant interference in both competitive and sandwichimmunoassays and remains an often overlooked confounder despite recently increased awareness.

Methods: We evaluated the interference of high dose biotin on time-critical electrochemiluminescence immunoassays like troponin Ths, PCT, TSH, beta-HCG, digitoxin, tacrolimus, cyclosporin A and others via controlled sample spiking as well as the effectiveness of an easy to perform countermeasure on Roche's cobas $® 8000$ modular analyzer series.

Results: Almost all tested immunoassays suffered from statistically and clinically relevant interference, potentially leading to wrong diagnoses or conclusions about the patient's physical condition. The evaluated procedure to eliminate biotin from the samples appears to be safe and produced reliable results, which were identical or very similar to the original measurements before spiking occurred.

Conclusion: Biotin is a significant confounder in many immunoassays. To avoid the interference, countermeasures which eliminate biotin from the samples or the use of biotin-independent assays are viable solutions. 


\title{
Endokrinologie, Stoffwechsel und Herz/Kreislauf -1
}

\author{
PO-16 / FV-01
}

\section{Proteomic profiling in heart failure patients with preserved ejection fraction: results from the Aldo-DHF trial}

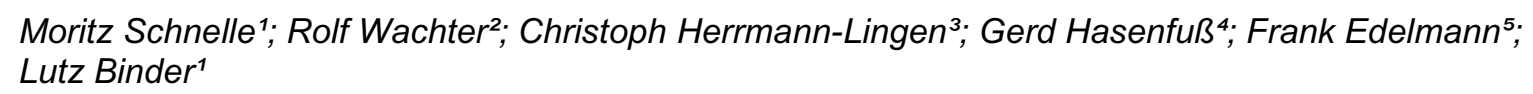

'Universitätsmedizin Göttingen, Institut für Klinische Chemie, Göttingen, Germany

${ }^{2}$ Universitätsklinikum Leipzig, Klinik und Poliklinik für Kardiologie, Leipzig, Germany

3Universitätsmedizin Göttingen, Psychosomatische Medizin und Psychotherapie, Göttingen,

Germany

${ }^{4}$ Universitätsmedizin Göttingen, Kardiologie und Pneumologie, Göttingen, Germany

${ }^{5}$ Charité-Universitätsmedizin Berlin, Innere Medizin und Kardiologie, Berlin, Germany

Objective: Heart failure (HF) with preserved ejection fraction (HFpEF) is a highly prevalent clinical condition accounting for up to $50 \%$ of all HF cases. Despite its relevance, only few advances in therapeutic strategies targeting HFpEF progression have been made. The Aldo-DHF trial recently demonstrated that spironolactone treatment can exert beneficial effects in HFpEF patients as indicated by improved diastolic function and reduced NT-proBNP levels. By using a proteomic approach, the purpose of this study was (i) to identify novel mechanisms which could mediate cardioprotective effects in HFpEF and (ii) to link protein expression with distinct clinical parameters in HFpEF patients.

Methods: 92 proteins, all linked to cardiovascular pathophysiologies, were measured in plasma samples of $380 \mathrm{HFpEF}$ patients from the multicentre, randomized, placebo-controlled Aldo-DHF trial. This included measurements at baseline $(n=380)$ and 12 months after spironolactone $(n=192)$ or placebo treatment $(n=185)$, followed by statistical analyses.

Results: Expression levels of six proteins were found to have significant interactions between the spironolactone and the placebo group over time: REN (renin), AMBP (a1-microglobulin/bikunin precursor), BNP (brain natriuretic peptide), MMP-7 (matrix metalloproteinase-7), GH (growth hormone) and VEGFD (vascular endothelial growth factor D). Linear regression models were used to correlate baseline plasma expression levels of the 92 measured proteins with five selected clinical parameters: 1. peak VO2 (exercise capacity), 2. E/e' (left ventricular diastolic function), 3. LVMI (left ventricular mass index), 4. LAVI (left atrial volume index) and 5. physical functioning reported on the SF-36 questionnaire. The highest number for significant correlations was found for SF-36 (46/92) followed by E/e' (18/92) and peak VO2 (8/92). For LAVI, only one protein was significantly correlated (BNP) and none was found for LVMI.

Conclusion: Spironolactone induces distinct protein expression changes in plasma of HFpEF patients. Some of these changes were not surprising, e.g. the increase in REN and the decrease in BNP. Others were unexpected such as the increase in MMP7 expression. Given the fact that MMP7 plays an important role in cardiac remodeling, this finding could be of pathophysiological relevance The high number of significant correlations between self-rated physical function and protein expression levels in our baseline analysis highlights the often unrecognized importance of perceived well-being in the setting of HFpEF. 


\section{PO-17 / FV-04}

The p21-mediated and senescence driven hyperglycemic memory in diabetic nephropathy is therapeutically amendable

Silke Zimmermann"; Moh'd Mohanad Al-Dabet²; Khurrum Shahzad²; Ahmed Elwakiel2; Rajiv Rana; Dheerendra Gupta2; Peter P. Nawroth³; Berend Isermann²

1Otto-von-Guericke-Universität Magdeburg, Medizinische Fakultät, Institut für Klinische Chemie und Pathobiochemie, Magdeburg, Germany

${ }^{2}$ Universitätsklinikum Magdeburg, Institut für Klinische Chemie und Pathobiochemie, IKCP, 39120 Magdeburg, Germany

${ }^{3}$ Universitätsklinikum Heidelberg, Klinik für Endokrinologie, Stoffwechsel und Klinische Chemie, Heidelberg, Germany

\section{Background:}

Diabetic nephropathy (dNP) is the leading cause of end-stage renal diseases (ESRD) in Europe. Tubulointerstitial damage has been shown to be a determinant for the attrition of renal function. The progression of diabetic complications, despite improvement of hyperglycemia can lead to long-lasting renal effect. This phenomenon, which is referred to as "hyperglycemic memory", is still poorly understood. Evidence suggests a key role of epigenetic mechanisms (DNA methylation, histone modifications), regulating the hyperglycemic memory. Whether SGLT2i reverse this memory, remains inconclusive. In dNP, the tubular compartment undergoes a particular pattern of damage characterized by induction of CDKi (mainly p21) leading to proliferative arrest, tubular hypertrophy, and senescencelike phenotype. Chronic senescence-state would hamper elimination of damaged cells; hence hinder tissue remodeling and repair. Further in vitro an in vivo work suggested that $p 21$ contributes to the hyperglycemic memory in mice (poster by Elwakiel et al).

Aim: We now want to investigate the role of p21in human dNP. Furthermore we want to show p21 as a potential diagnostic marker for dNP. Methods and materials:

We performed stainings (IFC, IHC) on human kidney sections for p21 and the senescence marker $\mathrm{yH} 2 \mathrm{~A} . \mathrm{X}$. We checked the expression of p21 in urine samples collected from patients with dNP and other CKDs by western blot. The urine underwent a procedure of centrifugation steps, leading to the isolation of a fraction containing macrovesicles. Results: Immunostaining revealed sustained tubular p21 expression and senescence specifically in kidney sections of diabetic patients with dNP in comparison to kidney sections from diabetic patients without dNP. We analyzed the presence of p21 in urine of controls and diabetic patients without and with dNP. dNP patients were recruited from a study in which a subgroup of patients was randomly assigned to a diet to lower blood glucose: Expression of p21 was elevated in the urine of dNP patients and remained high despite reduction of blood glucose levels upon intermittent diet. Conversely, no p21 expression was detected in the urine of healthy controls. Furthermore, we analyzed urine samples of diabetic patients from an independent cohort, compared these to patients with other CKDs. Urinary p21 levels were only elevated in dNP patients, not in those with other CKDs. We are now evaluating a third cohort from the LIFE-study. Conclusion: Our results indicate that the renal induction of p21 is a specific hallmark of diabetic nephropathy in humans. p21 expression in tubular cells is associated with a senescence-like phenotype in human renal sections. Urinary p21 levels identifies patients with dNP and remains high despite improving of blood glucose, indicating that urinary p21 is a marker of the renal metabolic memory. 


\section{PO-18 / PV-03}

The p21-mediated and senescence-associated hyperglycemic memory in diabetic nephropathy is therapeutically amendable

Ahmed Elwakiel'; Moh'd Mohanad Al-Dabet'; Khurrum Shahzad'; Alba Sulaj; Stefan Kopfi; Ihsan Gadi2; Rajiv Rana'; Gupta Dheerendra²; Silke Zimmermann²; Peter Mertens'; Peter P. Nawroth ${ }^{3}$; Christopher Dockendorff; Shrey Kohli; ; Berend Isermann²

'Otto-von-Guericke-Universität Magdeburg Medizinische Fakultät, Institut für Klinische Chemie und Pathobiochemie, Magdeburg, Germany

${ }^{2}$ Medizinische Fakultät / Universitätsklinikum Otto-von-Guericke-Universität, Institut für Klinische Chemie und Pathobiochemie, Magdeburg, Germany

${ }^{3}$ Universitätsklinikum Heidelberg, Klinik für Endokrinologie, Stoffwechsel und Klinische Chemie, Heidelberg, Germany

${ }^{4}$ Medizinische Fakultät / Universitätsklinikum Otto-von-Guericke-Universität, Klinik für Nephrologie, Diabetologie, and Endocrinologie, Magdeburg, Germany

${ }^{5}$ Marquette University, Department of chemistry, Milwaukee, United States

${ }^{6}$ Universitätsklinikum Leipzig, Institut für Laboratoriumsmedizin, Klinische Chemie und Molekulare Diagnostik, Leipzig, Germany

Background: Diabetic nephropathy (dNP), which is a major chronic microvascular complication among diabetic patients, is the leading cause of end stage renal disease (ESRD) worldwide. dNP is characterized by albuminuria and / or declining glomerular filtration rate (GFR) and histomorphological changes resulting in ESRD. A major therapeutic obstacle in dNP is the failure of renal recovery upon improved blood glucose levels. The mechanisms underlying this phenomenon, known as the hyperglycemic memory, remain unknown.

Aim: We aimed to identify mechanisms and therapeutically amendable targets contributing to the hyperglycemic memory in dNP.

Methods and materials: Two mouse models with established dNP (16 weeks after STZ-induced persistent hyperglycemia or 16 weeks old db/db mice) were used in the study. Blood glucose was reduced for 6 weeks using an SGLT2-inhibitor, mimicking therapy in diabetic patients. An unbiased approach (mRNA-seq) was used to evaluate pathways involved in hyperglycemic memory. In vitro and in vivo studies were conducted to determine mechanistic and translational relevance.

Results: Despite a marked reduction of blood glucose levels using SGLT2 inhibition, albuminuria, histomorphological changes, and glucose induced altered renal gene expression persisted. Functional annotation of persistently changed gene expression revealed that PI3-kinase-Akt signaling, cellular proliferation and senescence, and complement and coagulation cascades were linked with hyperglycemic memory. Sustained expression of p21, a senescence-associated cyclin-dependent kinase inhibitor, was among the top hits. Sustained tubular expression of p21 despite blood glucose lowering was confirmed in diabetic mice (histology, RNA and protein). Sustained p21 expression was linked with demethylation of its promoter and reduced DNA methyl transferase (DNMT) activity and DNMT1 expression. The nephroprotective zymogen protein $C$ was among genes persistently repressed in dNP. Increased tubular senescence, interstitial fibrosis, and albuminuria were confirmed in diabetic mice with genetically superimposed impaired protein $C$ activation. A role of miR-148a, identified in silico as a potential regulator of DNMT1, was confirmed in tubular cells in vitro. Substituting the protease activated protein $\mathrm{C}(\mathrm{PPC})$, mimicking biased aPC-signaling (parmodulin-2), or reducing miR-148a in addition to normalizing blood glucose levels reversed sustained tubular p21 expression, tubular senescence, and renal damage in diabetic mice with already established dNP.

Conclusion: Epigenetically sustained p21expression and associated senescence contribute to the hyperglycemic metabolic memory in dNP. This pathogenic mechanism can be targeted by inhibiting miR-148a or by mimicking cytoprotective aPC-signaling. 
PO-19

Children and adolescents with obesity have reduced serum bone turnover markers and 25hydroxyvitamin D but increased PTH concentrations - results derived from new age- and gender-dependent pediatric reference ranges

Mandy Geseric'; Mandy Vogel'; Felix Eckelt; Maike Schlingmann"; Andreas Hiemisch'; Ronny

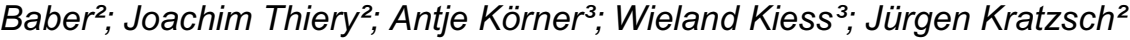

${ }^{1}$ Universitätsklinikum Leipzig, LIFE - Leipzig Research Center for Civilization Diseases, Leipzig, Germany

Universitätsklinikum Leipzig, Institute for Laboratory Medicine, Clinical Chemistry and Molecular Diagnostics (ILM), Leipzig, Germany

${ }^{3}$ Universitätsklinikum Leipzig, Department of Women and Child Health, Hospital for Children and Adolescents and Center for Pediatric Research (CPL), Leipzig, Germany

Background: We aimed to establish age- and gender-specific reference ranges for concentrations of the bone markers osteocalcin (OC), procollagen type $1 \mathrm{~N}$-propeptides (PINP) and carboxy-terminal cross-linking telopeptide of type 1 collagen (CTX-I) as well as for the calciotropic hormones 25hydroxyvitamin $\mathrm{D}[25(\mathrm{OH}) \mathrm{D}]$ and parathyroid hormone $(\mathrm{PTH})$ in healthy infants, children and adolescents. In addition, the effect of age, gender, puberty and body mass index (BMI) on bone markers was investigated.

Methods: 2416 healthy subjects (5714 blood withdrawals), aged 3 months to 17 years, were included to estimate the age- and gender-dependence of reference ranges. Subsequently, measured values of the biomarkers were transformed to standard deviation scores (SDS) and their associations with age, gender and puberty were analyzed. Bone marker-SDS values of the reference cohort were compared with an obese cohort ( $\mathrm{n}=317$ and 489 blood withdrawals) to analyze the effect of BMI.

Results: OC, PINP and CTX-I showed a distinct age- and gender-dependence with peak levels at 10 to 11 years (girls, Tanner 3 ) and 13 years (boys, Tanner 3-4). Children with obesity had significantly lower SDS levels for OC (-0.44), PINP (-0.27), CTX-I (-0.33), 25(OH)D (-0.43) and higher SDS levels for PTH (+0.37) than the reference cohort.

Conclusions: OC, PINP and CTX-I vary with age, gender and pubertal stage. The body weight status has to be considered in the interpretation of pediatric OC, PINP, CTX-I, 25(OH)D and PTH levels. Consequences of childhood obesity on bone health should be carefully investigated in long-term studies. 


\title{
PO-20
}

\section{Troponin I and T in hospitalized patients: Inconsistant findings in suspected myocardial infarction}

\author{
Abass Eidizadeh"; Laura Fraune'; Andreas Leha'; Lutz Binder ${ }^{1}$ \\ 'Universitätsmedizin Göttingen, Institute for Clinical Chemistry/Interdisciplinary UMG-Laboratory, \\ Göttingen, Germany \\ 'Universitätsmedizin Göttingen, Department of Medical Statistics, Göttingen, Germany
}

\section{Background:}

Cardiac troponins are crucial for the diagnosis of acute myocardial infarction. Commercial assays for the measurement of the two isoforms, cardiac troponin I (cTnl) and cardiac troponin T (cTnT) are since long available. Despite known differences in their diagnostic significance there are no recommendations for only one of the two isoforms so far.

In an everyday diagnostic setting of a university hospital, we compared the two cardiac troponin isoforms in patients where the attending physician ordered troponin measurement. We investigated the number of identical or discrepant findings of the troponins and tried to identify causes for the different results.

\section{Methods:}

Within one year, cTnT (Cobas e411, Roche) as well as cTnl (Architect-i1200, Abbott) were measured in all troponin-requested samples in the interdisciplinary laboratory of the University Medical Center Göttingen $(n=5667)$. Furthermore, at least one creatinine concentration per patient case (Architectc1600, Abbott) was determined. The comparability of both isoforms was checked by crosstab using the decision limits specified by the manufacturers. In the case of $\mathrm{cTnl}$, both gender-dependent and gender-independent cutoffs were considered. For cTnT, no gender-specific cutoffs were given by the manufacturer. With the help of ICD-10 coded diagnoses from the DRGs, ROC analyzes were carried out.

Results:

In a direct comparison, clear discrepancies between the two isoforms were found. In particular, the number of cTnT positive and cTnl negative results was strikingly different. Even after elimination of patients with an eGFR $<60 \mathrm{ml} / \mathrm{min} / 1.73 \mathrm{~m}^{2}$, and when using sex-dependent cTnl cutoffs, this group was considerably greater than the group with cTnl positive and cTnT negative results.

Comparing the troponin cases with a dynamic increase or decrease between two measurements, the number of discrepant cases in both groups were balanced. In addition, the sensitivity and specificity of cTnl was increased when using sex-dependent cutoffs.

\section{Conclusion:}

This study shows that the two cardiac troponin isoforms are not considered to result in identical analytical results and thus may favour discrepant clinical diagnoses and decisions.

Sex-dependent cutoffs may considerably improve sensitivity and specificity of the test. 


\title{
PO-21
}

Analysis of free steroid hormones by temperature controlled ultrafiltration and subsequent LCMS/MS: A pilot study

\author{
Martin Külz'; Jürgen Kratzsch; Uta Ceglarek; Alexander Gaudl'; Yoon Ju Bae1 \\ ${ }^{1}$ Institut für Laboratoriumsmedizin, Klinische Chemie und Molekulare Diagnostik Leipzig, Leipzig, \\ Germany \\ ${ }^{2}$ Institut für Laboratoriumsmedizin, Klinische Chemie und Molekulare Diagnostik Leipzig, \\ Immunologische Diagnostik, Endokrinologische Diagnostik, Leipzig, Germany \\ ${ }^{3}$ Institut für Laboratoriumsmedizin, Klinische Chemie und Molekulare Diagnostik Leipzig, Bereich \\ Forschung, Molekulare Diagnostik, Spezialdiagnostik, Leipzig, Germany \\ ${ }^{4}$ Institut für Laboratoriumsmedizin, Klinische Chemie und Molekulare Diagnostik Leipzig, Bereich \\ Forschung, Massenspektrometrie, Leipzig, Germany
}

Background: Steroid hormones are associated to binding proteins in peripheral blood. According to the "free hormone hypothesis", only the free hormone molecules are biologically active. Currently, the free fractions are commonly calculated from the total hormone content as well as from the levels of binding proteins. The aim of our study was to develop a method composed of ultrafiltration and LC-MS/MS for the measurement of free testosterone (SFT) and free cortisol (SFC) in human serum.

Methods: Preheated sera were prepared by ultrafiltration at $37 \pm 1^{\circ} \mathrm{C}$ with constant temperature monitoring. Following ultrafiltration, the potential free hormone fraction was measured via LC-MS/MS (A. Gaudl et al. 2016). In the pilot study, we measured SFT and SFC in 70 randomly selected samples from clinical routine diagnostics. SFT results were compared to calculated values (SFTcalc) and SFC results were compared to measured salivary free cortisol values (SalFC). Within-run imprecision including the ultrafiltration step was below $12.2 \%$.

Results: The measurement of SFT in female patients was restricted due to insufficient sensitivity of the analytical method. In male and transgender patients $(n=31$, age 14 - 68 years), we determined (mean $\pm S D$ ) $188 \pm 160 \mathrm{pmol} / \mathrm{L}$ for SFT and $295 \pm 211 \mathrm{pmol} / \mathrm{L}$ for SFTcalc in the direct comparison. The coefficient of correlation was $r=0.91(p<0.001)$. SFC levels were $8.16 \pm 4.73 \mathrm{nmol} / \mathrm{L}$ compared to $6.68 \pm 15.3 \mathrm{nmol} / \mathrm{L}$ for SalFC. The correlation of SFC and SalFC was $r=0.58(n=18, p<0.05)$. The correlation of total serum levels of cortisol with SFC was $r=0.89(p<0.001)$ and therefore distinctly higher than the correlation with SalFC $(r=0.28 ; p<0.01)$.

Conclusion: The cause for the differences in the results of SFT vs. SFTcalc and of SFC vs. SalFC has to be elucidated in further studies including a distinctly higher number of clinically detailed characterized patients. Results of such studies should enable us to decide, if free hormone values measured by ultrafiltration have a better diagnostic relevance than currently used calculation or saliva procedures. 


\title{
PO-22
}

\section{Free and bioavailable 25-hydroxyvitamin $D$ in normal human gestation}

\author{
Oleg Tsuprykov'; Claudia Buse²; Roman Skoblo³; Berthold Hocher ${ }^{4}$ \\ ${ }^{1} / F L b$ Laboratoriumsmedizin Berlin GmbH, Molekularbiologie, Berlin, Germany \\ 2IFLb Laboratoriumsmedizin Berlin GmbH, Klinische Chemie, Berlin, Germany \\ ${ }^{3} I F L b$ Laboratoriumsmedizin Berlin GmbH, Geschäftsführung, Berlin, Germany \\ ${ }^{4}$ Hunan Normal University, Key Laboratory of Study and Discovery of Small Targeted Molecules, \\ Changsha, China
}

Vitamin $D$ deficiency is highly prevalent in pregnant women and is associated with an increased risk of developing pregnancy complications. 25-hydroxyvitamin $D(25 \mathrm{OHD})$ is considered the best marker of vitamin D status so far. Serum $250 H D$ is composed of vitamin D-binding protein (DBP)-bound fraction $(85 \%)$, albumin-bound fraction $(15 \%)$, and a tiny amount of a fully unbound, free fraction $(<0.1 \%)$, called "free 25OHD" (25OHDf). The sum of free and loosely albumin-bound $250 \mathrm{OH}$ metabolites is called "bioavailable 25OHD" (25OHDb). Total 25OHD (25OHDt) - current vitamin D status routine marker - is the sum of all 25OHD metabolites. Due to estrogen-influenced DBP elevation during pregnancy, $25 \mathrm{OHDt}$ might not adequately reflect vitamin $\mathrm{D}$ status. We aimed to compare the correlation strength between serum 250HDt, 25OHDf, and 25OHDb and biomarkers of bone health, lipid metabolism and kidney function in normal human gestation.

The study was carried out in 427 pregnant healthy women residing in Berlin, Germany. Study was performed in late spring. 250HDt was determined by Abbott Architect i2000 platform. DBP was measured by an ELISA (Immundiagnostik, Germany). 25OHDb and 25OHDf were calculated using the formula based on the levels of 25OHDt, DBP and albumin. Spearman rank correlation analysis (Spearman's rho $(\rho))$ was used.

All 25OHD metabolites showed a negative correlation of a nearly equal strength with PTH $(\rho=-0.292$; $\rho=-0.266 ; \rho=-0.256$ for 25OHDt, $25 \mathrm{OHDf}$, and $25 \mathrm{OHDb}$, respectively, $p<0.001$ for all). However, serum calcium correlated with 25OHDf and $25 \mathrm{OHDb}(\rho=0.234$ and $\rho=0.369$, respectively, $p<0.001$ for all), but not with 25OHDt $(\rho=0.080, p=0.098)$. All 25OHD isoforms correlated with bone alkaline phosphatase, however the association strength was slightly higher with free and bioavailable 25OHD isoforms compared to 25OHDt $(\rho=-0.155, p=0.001 ; \rho=-0.253, p<0.001 ; \rho=-0.287, p<0.001$ for 25OHDt, 25OHDf, and 25OHDb, respectively). Low-density lipoprotein correlated with 25OHDf and 25OHDb, but not with 25OHDt $(\rho=-0.174, p<0.001 ; \rho=-0.300, p<0.001 ; \rho=0.049, p=0.315)$. In contrast, only 25OHDt showed significant associations with high-density lipoprotein $(\rho=0.228, p<$ 0.001 ). Adiponectin correlated with 25OHDf and 25OHDb $(\rho=0.140, p=0.005$ and $\rho=0.168, p=$ 0.001 for $25 \mathrm{OHDf}$ and $25 \mathrm{OHDb}$, respectively), but not with 25OHDt $(\rho=0.063, p=0.202)$. Only $25 \mathrm{OHDf}$ and 25OHDb $(\rho=0.161, p=0.001$ and $\rho=0.240, p<0.001)$, but not 25OHDt $(\rho=0.026, p$ $=0.597$ ) significantly correlated with urea. In addition, red blood cell count and hemoglobin showed a significant (positive) correlation only with 25OHDf and 25OHDb, but not with 25OHDt.

Both free and bioavailable 25OHD showed overall better associations with markers of bone health, lipid metabolism and kidney function than routine total 25OHD. Thus, optimal monitoring of vitamin $D$ status in pregnancy, in addition to $25 \mathrm{OHDt}$, should include either $25 \mathrm{OHDf}$ or $25 \mathrm{OHDb}$ determination. 
PO-23

\section{Effects of a lifestyle intervention on the hemostasis system of individuals with prediabetes}

Sebastian Hörber"; Rainer Lehmann"; Louise Fritsche²; Norbert Stefan ${ }^{3}$; Martin Heni; Andreas Fritsche $^{3}$; Andreas Peter ${ }^{1}$

${ }^{1}$ Universitätsklinikum Tübingen, Institut für Klinische Chemie und Pathobiochemie, Tübingen, Germany

${ }^{2}$ Universitätsklinikum Tübingen, Institut für Diabetesforschung und Metabolische Erkrankungen (IDM) des Helmholtz Zentrums München an der Universität Tübingen, Tübingen, Germany ${ }^{3}$ Universitätsklinikum Tübingen, Innere Medizin IV - Diabetologie, Endokrinologie und Nephrologie, Tübingen, Germany

Background: Prediabetes (impaired glucose tolerance (IGT) and impaired fasting glucose, IFG)) and obesity are associated with a prothrombotic state and increased risk to develop cardiovascular disease. Lifestyle intervention has been shown to be effective in reducing body weight and preventing or at least postponing the manifestation of type 2 diabetes and associated sequelae. Our aim was to examine the effect of a lifestyle intervention on the hemostasis system in individuals with IGT/IFG.

Study design and methods: 100 individuals with IGT/IFG and high-risk to develop type 2 diabetes were included in the study. Citrated blood samples were obtained at baseline and after one year of a standardized high intensive lifestyle intervention program. Hemostasis testing included the measurement of prothrombin time (PT), activated partial thromboplastin time (aPTT), activities of coagulation factors, von-Willebrand factor (VWF) activity, fibrinogen and D-dimer concentrations on the Atellica COAG 360 coagulation analyzer (Siemens Healthcare).

Results: Hemostasis measurements revealed significantly reduced levels for PT, coagulation factors II, VII, VIII, IX, XI, XII and vWF activity at follow-up visits compared to baseline results. Fibrinogen, Ddimer and FXIII showed no change during the first year.

Conclusion: This is a preliminary evaluation of hemostasis measurements during a lifestyle intervention program of individuals with IGT/IFG. We can demonstrate that this is effective in reversing the prothrombotic state and thereby may contribute to a reduced cardiovascular risk. Further analyses will focus on the underlying mechanism including anthropometric data, metabolic parameters and body fat composition. 


\section{Endokrinologie, Stoffwechsel und Herz/Kreislauf -2}

\section{PO-24}

\section{Biomarker assessment of insulin resistance and severity of polycystic ovary syndrome}

Josef van Helden'; Osman Evliyaoglu²; Ralf Weiskirchen²

'Labor Mönchengladbach MVZ Dr. Stein und Kollegen, Analytik, Mönchengladbach, Germany ${ }^{2}$ Uniklinik RWTH Aachen, Institut für Molekulare Pathobiochemie, Experimentelle Gentherapie und Klinische Chemie, Aachen, Germany

Objective: To explore the relationship between insulin resistance in women with PCO syndrome and remaining ovarian function in order to predict the likelihood of conception. Different PCOS phenotypes are correlated with different clinical severity levels. Insulin resistance correlates with higher severity. The parameters glucose, insulin, insulin $60 \mathrm{~min}$ after intake of $75 \mathrm{~g}$ glucose and adiponectin or sAMHR2 enable a biochemical classification of PCO patients that correlates with morphological PCO phenotypes..

Methods: In a retrospective study, 103 patients with polycystic ovary syndrome were examined for insulin resistance over a period of two years. Weight, height, cycle day, cycle length were obtained, fasting glucose, insulin 0,60 and 120 min after $75 \mathrm{~g}$ glucose intake, c-peptide, intact proinsulin, adiponectin and CRP were measured for diagnostic purposes, the HOMA- IR was calculated. AMH and SAMHR2 were measured as additional parameters, the AMH - z-score and the sAMHR2 / AMHratio were calculated..

Results: A strong correlation between sAMHR2 and adiponectin $(r=0.818, p<0.0001)$ was found. As significant changes of biomarkers different impaired glucose metabolism conditions patients with insulin resistance and insulin overstimulation showed strong significant difference in sAMHR2, adiponectin levels, sAMHR2 / AMH ratio and HOMA-Index, proinsulin and BMI, AMH, AMH z-score and Insulin levels in $0-60-120$ Minute of glucose tolerance test.

Conclusions: By determining biomarkers it is possible to classify PCO patients into subgroups that correlate with different PCO phenotypes and the clinical severity. 


\title{
PO-25
}

\section{Age-specific Reference Values for the Roche Elecsys ${ }^{\circledR}$ AMH assay and its Diagnostic Performance in PCOS}

\author{
Josef van Helden"; Ralf Weiskirchen; Osman Evliyaoglu² \\ ${ }^{1}$ Labor Mönchengladbach MVZ Dr. Stein und Kollegen, Analytik, Mönchengladbach, Germany \\ ${ }^{2}$ Uniklinik RWTH Aachen, Institut für Molekulare Pathobiochemie, Experimentelle Gentherapie und \\ Klinische Chemie, Aachen, Germany
}

Objective: To study the age-specific reference values of anti-Müllerian hormone and describe the diagnostic performance in polycystic ovary syndrome. The important influence of PCOS on reproductive, obstetric, metabolic and cardiovascular diseases makes the early detection of this syndrome particularly important in terms of a higher clinical and prognostic value (7). In July 2018, the evaluation of guidelines on PCOS considered a single serum marker, such as serum AMH, inadequate to meet the diagnostic criteria because of the wide heterogeneity and complexity of the disease.

Methods: This cross-sectional study was conducted between 2016 and 2019 in a single reference laboratory in Germany. A total of 4175 reproductive age patients, ranging from 14 to 50 years from various gynaecological practices and fertility centers in Northrhine Westfalia, Germany, were included. . Reference values were given as means and standard deviations (SD) or \% percentiles. We calculated Youden's Index (sensitivity + specificity - 1) to determine the age-specific optimal limits for each parameter. Receiver operating characteristics (ROC) were created to examine the diagnostic value of $\mathrm{AMH}, \mathrm{FAl}$ and $\mathrm{LH} / \mathrm{FSH}$ ratio.

Results: The age-specific reference values of patients with normal cycle were calculated as mean \pm standard deviation values and percentiles (\%) values of the Elecsys $®$ AMH Test. Furthermore, the diagnostic performance for polycystic ovary syndrome was calculated of the parameters for each age group. In the ROC analysis, the AUC, FAI and LH / FSH ratios of AMH were 0.977, 0.773 and 0.786 , respectively. For each parameter, cut-off levels, and the values having $100 \%$ sensitivity or $100 \%$ specificity were determined. Furthermore, we calculated the diagnostic performance for polycystic ovary syndrome of the parameters for each age group.

Conclusion(s): AMH declines, indicating changes of ovarian maintenance. AMH showed the best performance in diagnosing PCOS. 


\section{PO-26}

\section{Associations of circulating chemerin and adiponectin concentrations with hepatic steatosis}

Stephanie Zylla'; Lena-Maria Levin'; Henry Völzke²; Markus M. Lerch ${ }^{3}$ Jens-Peter Kühn ${ }^{4}$; Matthias Nauck ${ }^{1}$; Nele Friedrich ${ }^{1}$

${ }^{1}$ Universitätsmedizin Greifswald, Institute for Clinical Chemistry and Laboratory Medicine, Greifswald, Germany

2University Medicine Greifswald, Institute for Community Medicine, Greifswald, Germany

${ }^{3}$ University Medicine Greifswald, Department of Medicine A, Greifswald, Germany

${ }^{4}$ University Medicine Greifswald, Department of Diagnostic Radiology and Neuroradiology, Greifswald, Germany

Objective: Chemerin and adiponectin are adipokines, which have been assumed to be involved in the development of metabolic syndrome-related phenotypes like hepatic steatosis. We aimed to evaluate the associations of circulating chemerin and adiponectin concentrations with liver enzymes, liver fat content, and hepatic steatosis in the general population.

Methods: Data of 3951 subjects from the population-based Study of Health in Pomerania (SHIPTREND) were used. Hepatic steatosis was assessed by the presence of a hyperechogenic liver (defined via ultrasound) or a magnetic resonance imaging (MRI)-quantified liver fat content $>5 \%$. Adjusted sex-specific quantile and logistic regression models were applied to analyze the associations of chemerin and adiponectin with liver enzymes, liver fat content, and hepatic steatosis.

Results: Concerning the associations of chemerin and adiponectin with liver enzymes, we observed very divergent findings depending on sex, fasting status, and the specific enzyme. More distinct results can be seen in the analyses of MRI-quantified liver fat content with strictly inverse associations to adiponectin in both sexes and a positive or U-shaped association to chemerin in men or women, respectively. Similarly, the MRI-based definition of hepatic steatosis revealed the most obvious results: in both sexes high chemerin concentrations were associated with higher odds whereas high adiponectin concentrations were associated with lower odds of hepatic steatosis.

Conclusions: We assume a potential role of these adipokines in the pathogenesis of hepatic steatosis independent of metabolic or inflammatory disorders, but further experimental studies are necessary to clarify the mechanism underlying the relation between adipokine concentrations and hepatic steatosis. 
PO-27

Advancement in steroid hormone analysis by LC-MS/MS in clinical routine diagnostics - a three year recap from serum cortisol to dried blood 17a-hydroxyprogesterone

\author{
Alexander Gaudl'; Jürgen Kratzsch ${ }^{1}$; Uta Ceglarek ${ }^{1}$ \\ ${ }^{1}$ Universitätsklinikum Leipzig, Institut für Laboratoriumsmedizin, Klinische Chemie und Molekulare \\ Diagnostik, Leipzig, Germany
}

Steroid analysis by LC-MS/MS in daily clinical routine diagnostics requires high-throughput conditions including fast chromatographic separation. Hereby, signal interferences may occur due to limited specificity in complex biologic matrices. During the past three years of routine steroid analysis in our laboratory and roughly 50,000 measurements, about $1 \%$ was affected by interferences, mainly serum cortisol (>90\% of the affected) and dried blood 17a-hydroxyprogesterone (17-OHP). To overcome specificity problems, enhanced chromatography, ionization polarity switching, and detection via twostage fragmentation (MS3) using a quadrupole linear ion trap were investigated in our study. Signal interferences of serum cortisol were eliminated by applying a protocol for automated method switching without changing the basic high-throughput LC-MS/MS setup. This approach includes negative ionization and extended chromatography from 4 to 6.6 minutes using the fourfold column length. From 9 samples affected by cortisol interference using the high-throughput method, 8 could be reliably analyzed applying the method switching protocol. Moreover, the applicability of the high-throughput method as second tier analysis in congenital adrenal hyperplasia $(\mathrm{CAH})$ diagnostics from dried blood was verified with $100 \%$ diagnostic specificity. In addition, the combination of fast LC and MS3 detection enables specific quantitation of 17-OHP from dried blood spots on a screening time scale. This approach may be an alternative to the newborn screening for CAH by immunoassay due to its higher specificity, reducing the number of false positive results by $90 \%$. In this work we recap experiences from three years of clinical routine steroid analysis via LC-MS/MS and present a unique analytical setup that enables both high-throughput and enhanced resolution analysis of steroid hormones in serum and dried blood. 


\title{
PO-28
}

\section{Proteome analysis of endothelial specific Ptp1b-knockout in pressure overloaded mice heart indicates improved mitochondrial function}

\author{
Sidra Shahid ${ }^{1}$; Marlena Pantakani2; Krishna Pantakani'; Lutz Binder ${ }^{2}$; Abdul-Rehman Asif ${ }^{2}$ \\ 'Universität Göttingen, Institute of Clinical Chemistry, Göttingen, Germany \\ 2UMG Göttingen, Institute for Clinical Chemistry, Göttingen, Germany
}

Cardiovascular diseases including, cardiac hypertrophy, atherosclerosis and coronary artery disease, are among the major cause of fatalities worldwide. Improving cardiac angiogenesis and functionality of the cardiovascular network are promising approaches after myocardial infarction. Receptor tyrosine kinases (RTKs) regulate signalling pathways after binding to angiogenic growth factors including VEGF, PDGF and FGF. Activation of RTKs is dependent on phosphorylation by tyrosine kinases while protein tyrosine phosphatases (PTPs) are negative regulators of RTKs. Protein Tyrosine Phosphatase-1B (PTP1B) is an ubiquitously expressed enzyme which targets Vascular Endothelial Growth Factor Receptor 2 (VEGFR2) and inhibits VEGF signal transduction. Multiple studies have shown that deletion of PTP1B improves angiogenesis and endothelial dysfunction. PTP1B expression is enhanced in high cardiac afterload mice models. PTP1B deletion and inhibition protects mice from cardiac hypertrophy, diabetes and fibrosis. However, ubiquitous expression of PTP1B in different tissues can be affected by global deletion or inhibition. To overcome this issue, a mouse model was generated with inducible endothelial cell specific PTP1B knockout (Endo.Ptp1b.KO). Transverse aortic constriction (TAC) was placed around the aorta to induce pressure on heart.

Endo.Ptp1b.KO mice showed reduced cardiac hypertrophy, fibrosis, oxidative stress and improved angiogenesis. Quantitative global proteome analysis using SWATH was performed on total heart lysates to identify possible pathways/proteins involved in improved cardiac function in Endo.Ptp1b.KO mice hearts. Wild type TAC-operated mice hearts exhibit overexpression of fibrosis associated proteins and reduced expression of mitochondrial proteins indicating mitochondrial dysfunction in WT mice hearts. In Endo.Ptp1b.KO mice hearts we also observed an increased expression of certain mitochondrial proteins related to electron transport chain and fatty acid metabolism. This indicates that Endo.Ptp1b.KO mice overcome cardiac overload by improving mitochondrial function.

This observation was further confirmed in our in-vitro model of Human Umbilical Cord-derived Vascular Endothelial Cells (HUVECs). Mitochondrial functional assays showed that PTP1B inhibition in HUVECS improved TNF- $\alpha$ induced mitochondrial dysfunction by increasing ATP production. The present study may help to better understand PTP1B function and to identify novel targets and approaches for CVD treatment. 
PO-29

\title{
Comparison of three different commercially available $L p(a)$ assays for clinical routine analysis
}

\author{
Benjamin Müller'; Jens Dreier²; Cornelius Knabbe ${ }^{2}$
}

${ }^{1}$ Herz- und Diabeteszentrum NRW, Universitätsklinik der Ruhr-Universität Bochum, Institut für Laboratoriums- und Transfusionsmedizin, Bad Oeynhausen, Germany ${ }^{2}$ Herz- und Diabeteszentrum Nordrhein-Westfalen, Universitätsklinik der Ruhr-Universität Bochum, Institut für Laboratoriums- und Transfusionsmedizin, Bad Oeynhausen, Germany

\section{Background:}

Lipoprotein (a) $[\mathrm{Lp}(\mathrm{a})]$ is highly suggested as an independent emerging risk factor in cardiovascular disease (CVD) prevention. With the progression of therapeutic possibilities, measurement of $L p(a)$ in routine laboratory is becoming more and more important. Lp(a) is a complex consisting of low-density lipoprotein (LDL) and an apolipoprotein B-100 particle linked to a plasminogen-like apolipoprotein(a) [apo(a)] via a disulfide bridge. This apo(a) part varies in size in each individual mainly due to variable numbers of the so-called kringle 4 type II domain, possibly affecting the result of $L p(a)$ measurement. The aim of the study was to compare the quantitation results of three different assays (Roche Tinaquant Lipoprotein (a) Gen. 2, Abbott QUANTIA Lp(a), DiaSys Lp(a) 21 FS) on routine samples.

Methods:

80 non-preselected leftover specimens from the clinical routine were tested with the three different assays (Abbott, Roche, DiaSys) on the ARCHITECT c8000 analyzer. Results were measured in $\mathrm{mg} / \mathrm{dL}$ and compared by linear regression using Passing-Bablok fit.

Results:

The quantification range of $\mathrm{Lp}(\mathrm{a})$ samples in $\mathrm{mg} / \mathrm{dL}$ for Abbott, DiaSys and Roche was 4.12 - 177.53, $4.93-187.19$ and 2.98 - 156.63 respectively. The method comparison by linear regression between the Roche and Abbott assays provided an intercept of 2,492 (1.966 to 2.739), a slope of 1.026 (1.003 to 1.064) with a correlation of $r=0.995$. The linear regression analysis between Abbott and DiaSys resulted in an intercept of $0.277(-0.224-0.862)$, a slope of 1.075 (1.047 to 1.131) with a correlation of $r=0.990$. The respective results for Roche and DiaSys method comparison were as follows: intercept 1.810 (1.135 to 2.166$)$, slope 1.190 (1.160 to 1.240$)$ with a correlation of $r=0.991$.

\section{Conclusion:}

$L p(a)$ can be measured with different methods on the same analyzer, showing similar results. The agreement between the results of the different methods is good across the measuring range under investigation, with best agreement seen between the Abbott and Roche assays. 
PO-30

\title{
Evaluation of Hemoglobin A1c-measurement by the reticulocyte count
}

\author{
Norbert Ostendorf'; Birgit Meyer-Noack ${ }^{2}$ \\ ${ }^{1}$ St. Katharinen-Hospital Frechen, Zentrallabor, Frechen, Germany \\ ${ }^{2}$ St. Katharinen-Hospital Frechen, Diabetologie, Frechen, Germany
}

Objective: HbA1c is commonly used for diagnosis and follow-up of Diabetes mellitus. The proportion of glycated $\mathrm{HbA} 1$ not only depends on the average blood glucose level, but also on erythrocyte lifespan. The latter is usually assumed to be 120 days, although in pathologic conditions like bleeding or iron deficiency anemia it can be largely different. In a retrospective study, we evaluated the dependency of $\mathrm{HbA} 1 \mathrm{c}$ results and reticulocyte count using routine laboratory data from an intermediate care hospital. To our knowledge, no study to date has investigated the potential role of reticulocyte counting to assess the reliability of $\mathrm{HbA} 1 \mathrm{c}$-values.

Methods: From 01.01.2009 to 31.05.2016, all hematologic samples sent to our laboratory for measurement of the reticulocyte count and/or proportion of $\mathrm{HbA} 1 \mathrm{c}$ were identified in the laboratory information system. In 297 cases, both parameters were measured simultaneously from the same blood sample. An additional number of 176 measurements were performed from different samples within no more than 5 days. All data sets were checked for comparability of hematologic parameters and for compromising incidents before or between measurements, like bleeding, transfusion or start of iron therapy. 47 data sets were excluded, leaving a total number of 426 . The data were anonymized and exported to $\mathrm{R}$ statistical program for further analysis.

$\mathrm{HbA1c}$ was measured using a competitive, immunoturbidimetric Assay (Beckman coulter Hemoglobin A1c) on a Beckman Coulter AU400 analyzer (Beckman Coulter, Krefeld, Germany). The reticulocyte count was performed on a Siemens hematology analyzer (ADVIA 120, Siemens Healthcare Diagnostics, Eschborn, Germany). In June 2016, the hematology analyzer was changed to a model from another manufacturer. Data collection was stopped at that point to avoid bias from the different assay.

Results: Using $35 \%$ reticulocytes as an arbitrary cut off, mean $\mathrm{HbA} 1 \mathrm{c}$ in the samples with a reticulocyte count below this threshold was $7.0 \%$ ( $53 \mathrm{mmol} / \mathrm{mol}, 373$ values, range 4.3 to $13.1 \%$ or 24 to $120 \mathrm{mmol} / \mathrm{mol})$. In the group with reticulocyte counts equal or greater than this cut off, mean $\mathrm{HbA} 1 \mathrm{c}$ was $6.3 \%$ ( $44 \mathrm{mmol} / \mathrm{mol}, 53$ values, range 4.4 to $16.1 \%$ or 25 to $152 \mathrm{mmol} / \mathrm{mol}$ ). Omitting the two highest values as outliers, the mean $\mathrm{HbA} 1 \mathrm{c}$ was $6 \%$.

Moreover, in 28 of the 473 measurements $(5.9 \%)$, the patients received red cell transfusions before $\mathrm{HbA1c}$ was analyzed. This is known to produce false low results.

Conclusion: Investigation of the reticulocyte count is a useful tool to evaluate the reliability of $\mathrm{HbA1C}$ measurements. Low or high reticulocyte counts indicate a bias on $\mathrm{HbA} 1 \mathrm{c}$ results.

In hospital routine, measurement of $\mathrm{HbA} 1 \mathrm{c}$ following red cell transfusion is not infrequent and must be taken into consideration when results are implausible. 


\title{
Molekulare Diagnostik
}

\section{PO-31 / PV-05}

\section{A fast and sensitive assay for detection of rejection after solid organ or stem cell transplantation}

\author{
Friederike Häuser'; Jens Mittler; Tanja Falter'; Markus Radsak²; Karl J. Lackner'; Heidi Rossmann ${ }^{1}$ \\ ${ }^{1}$ University Medical Center Mainz, Institute of Clinical Chemistry and Laboratory Medicine, Mainz, \\ Germany \\ ${ }^{2}$ University Medical Center Mainz, Department of General and Abdominal Surgery, Mainz, Germany \\ Objective
}

Haematopoietic chimerism and minimal residual disease trigger clinical decisions in patients after allogenic stem cell transplantation. After solid organ transplantation, for example LTx, informations about transplant engraftment and the differentiation between rejection and other conditions are also crucial. There is a need for comprehensive characterization of the molecular remission status after transplantation. We developed a fast and simple assay for the detection of molecular rejection markers (graft DNA) on a digital PCR platform.

Material and method

Graft cell-free DNA (Gcf-DNA) and leucocyte DNA of the peripheral blood was isolated from blood samples of patients undergoing solid organ transplantation or a haematopoietic stem cell transplantation respectively. All samples were screened for at least three informative gene loci (on $X$ or $\mathrm{Y}$ gene in patients with sex mismatched transplantation or common SNP with a MAF of 0.5 in patients with sex matched transplantation) by the digital droplet PCR (ddPCR) using QX200 (Bio-Rad).

\section{Results}

32 Taqman SNP assays and three assays targeting genes on the gonosomes were evaluated with a limit of detection of $0.1-0.99$ copies $/ \mathrm{ml}$ (0,02\% donor DNA). Samples of patients undergoing an allotransplantation were collected from the day after transplantation up to one year. The samples were screened for at least three informative gene loci and the donor fraction of DNA over time. An increase of GcfDNA is an indicator for rejection in solid organ transplanted patients as well as the increase of recipient's leukocyte DNA after stem cell transplantation.

\section{Discussion}

After receiving donor and recipient samples and the evaluation of the assay system, ddPCR turned out to be a simple and highly sensitive method for the early detection of transplant engraftment or rejection in patients, who underwent haematopoietic stem cell or solid organ transplantation. It has still to be evaluated, whether this method is superior to conventional methods for the identification of transplant rejection, as for example the assessment of classical biomarkers, MRD or STR-PCR. 


\title{
PO-32
}

\section{Chlamydia trachomatis infections in patients attended at ambulatory health units in Germany}

\author{
Philipp Göcke1; Carlos José Téllez-Castillo; Lisei Meining1; Claas Scharmann²; Silke Biermann-
} Göcke ${ }^{1}$

${ }^{1}$ Praxis für Labormedizin und Mikrobiologie, PCR, Bochum, Germany

${ }^{2}$ Praxis für Labormedizin und Mikrobiologie, Mikrobiologie, Bochum, Germany

Background: Chlamydia trachomatis (CT) infection is the commonest bacterial sexually transmitted infection (STI) in Europe. A lot of the patients infected do not show any symptoms. The infection can spread to the upper genital tract in women causing pelvic inflammatory disease. Untreated infections can cause a higher rate of abortions, low birth weight and transmission to the newborn. In Germany an estimated number of about 100,000 young women are involuntarily sterile due to infection by CT. Since diagnosis is made fast and an early detected infection can be treated easily, the screening of young men and women is an important part of health care.

Objective: We determined the percentage of positive polymerase chain reaction (CT-PCR) results and the epidemiological variables in women and men attended at ambulatory health units of the RhineRuhr metropolitan region in North Rhine-Westphalia, Germany.

Material and Methods: Retrospective study of infection with CT was performed from January 2015 to December 2018 in which 72103 clinical samples from outpatients were collected. 45215 (62.71\%) urine samples, $27(0.04 \%)$ semen samples and 26861 (37.25\%) swabs were tested with the CT/NG assay on the Abbott $\circledast$ m2000 RealTime system. Demographic and clinical characteristics of the patients (sex, age and pregnancy) were obtained from the patient records and included in the analysis. We considered one specimen per examination.

Results: 72103 specimen from 72103 patients were analysed during four years. A total of 67911 $(94.2 \%)$ clinical samples were collected from woman and $4192(5.8 \%)$ from men. A positive finding could be made in 2603 (3.6\%) of all samples in the four years of the study. Overall, the specimen collected from men where statistically significant more often tested positive $(n=524)$ than those collected from women $(n=2079)(p<0.05)$. The average age of CT positive women was 24.3 years and 28.4 years for the males, respectively. The highest rate of positive results in women was found in the group aged $15-19$ years $(6.9 \%)$. In the group of the male, the highest incidence of positive results $(26.8 \%)$ could be found in the group of the $20-24$ year old patients. At the moment of sample collection, $27219(40.1 \%)$ of all female patients were pregnant and $589(2.2 \%)$ of these showed a positive test result. The mean age of CT positive pregnant women was 24.7 years $(n=589)$.

Conclusions:

The CT prevalence found in our study is similar to prevalence reported by the RKI when they investigated a very large cohort with similar characteristics of german patients in 2013 .

Statistically significant more men than women were diagnosed with infection by CT.

The highest prevalence of positive results was observed in the group of 15-19 years of age for women and 20-24 years of age for males. 
PO-33

Xylosyltransferase (XT) deficient human embryonic kidney 293 (HEK293) cells are not viable

Bastian Fischer1; Thanh-Diep Ly'; Doris Hendig'; Joachim Kuhn"; Cornelius Knabbe ${ }^{1}$; Isabel Faust ${ }^{1}$

${ }^{1}$ Herz- und Diabeteszentrum Nordrhein-Westfalen, ILTM, Bad Oeynhausen, Germany

Background: Xylosyltransferases-I and -II (XT-I and XT-II) catalyze the rate-limiting step of proteoglycan (PG) biosynthesis. PG are abundant molecules within the extracellular matrix (ECM) and elevated XT-activities are associated with the manifestation of fibrosis. In all higher organisms exist two XT-isoforms, whereas other organisms only harbor one. The reasons for the appearance of two human XT-isoenzymes, which are tissue-specifically expressed, are not known and remarkable, as both proteins are very similar in their characteristics.

In our study we evaluated for the first time, whether decreased expressions of both $\mathrm{XT}$-isoforms have an impact on naturally XT-I deficient, immortalized HEK293 cells. We first conducted a siRNA XYLT2 knockdown and examined, whether mRNA expression levels, proliferation and viability of these cells are altered. To verify the results, we additionally conducted a stable CRISPR/Cas9 mediated XYLT2 knockout in HEK293 cells.

Methods: HEK293 cells were transfected with either a siRNA targeting the XYLT2 gene-product or a control siRNA, which does not target any human mRNA. 24, 72 and $96 \mathrm{~h}$ after conducting the knockdown, cell morphologies were controlled microscopically. Total RNA was isolated $48 \mathrm{~h}$ after transfection of the cells and gene expression levels of different ECM-associated targets were determined by quantitative realtime-PCR. For XYLT2 knockout, cells were transfected with a plasmid inter alia harboring a Cas9- and a cloned gRNA-sequence, binding within exon 2 or exon 9 of XYLT2. After separation of transfected cells, genomic DNA of proliferative cell-clones was isolated and analysed by sequencing. TA-cloning was performed, to allel-specifically characterize mutations.

Results: XYLT2 mRNA expression was highly significantly decreased after conducting the siRNAmediated XYLT2 knockdown, while determined gene expression levels of the ECM-associated targets ACAN, ELN and FN1 were all significantly increased. The knockdown also led to a decreased proliferation, abnormal morphologies and elevated cellular senescence of treated HEK293 cells. As a result of the CRISPR/Cas9-mediated XYLT2 knockout, cells showed reduced viability and TA-cloning revealed, that introduced mutations were very heterogenous. Beside frameshift-mutations, which generated premature stop-codons, proliferative cell-clones all harbored one in-frame mutation, leading to a shortened XT-II protein. XYLT2 mRNA levels of these proliferative cells were quite similar to controls.

Conclusion: HEK293 cells harboring at least one in-frame deletion, and therefore residual XT-II protein levels, were proliferative. Cells expressing diminished levels of both XT-isoenzymes showed decreased viability and elevated cellular senescence, clarifying that HEK293 cells cannot compensate for diminished expressions of both XT-isoforms. These results reveal important new findings concerning the relevance of both XT-isoenzymes for cell viability and proliferation. 


\section{PO-34} Home-made MLPA assays: valuable copy number screening tools in (rare) monogenic
diseases

Amir Jahic ${ }^{1}$; Christian Beetz ${ }^{2}$

${ }^{1}$ Charité-Universitätsmedizin Berlin, Institut für Laboratoriumsmedizin, Klinische Chemie und Pathobiochemie, Berlin, Germany

${ }^{2}$ CENTOGENE AG, Research and Development, Rostock, Germany

Introduction:

Copy number variations (CNVs) contribute to the mutational spectra in many monogenic disorders. As they are not detectable by standard PCR-based approaches, a number of alternative assays have been developed, with the Next Generation Sequencing (NGS) CNV-calling probably being the most promising approach. However, the multiplex ligation-dependent probe amplification (MLPA) is still the most frequently applied technique in routine diagnostics. MLPA-assays are usually obtained commercially. We were interested in whether home-made assays represent valid alternatives.

Methods:

We developed numerous home-made MLPA assays. They addressed various diagnostic, research, and methodical questions.

Results:

Our in house assays generate high quality data as concluded from high inter-sample and intra-sample homogeneity of MLPA signals. Known genetic aberrations present in positive control samples were unambiguously detected. MLPA-based evidence for CNVs was revealed in many additional samples and confirmed by independent approaches. Our findings contributed to several scientific publications.

Conclusion:

Home-made MLPA assays robustly detect large genomic CNVs. Limited multiplexing capacity ( 15fold compared to $\sim 50$-fold for commercial kits) is compensated by availability within days/weeks rather than months. An application is especially warranted when there is a little interest in the commercial development, i.e. in rare diseases or when the relevance of CNVs is not (yet) clear. Our laboratory approach will be made available to researches interested in more accurately defining the prevalence of CNVs in certain genes. 


\section{PO-35}

\section{Stitch and spit: Monitoring cell-free DNA in plasma and saliva improves early recurrence detection for head and neck cancer patients}

Romina Rösch"; Irina Kerle²; Markus Wirth ${ }^{3}$; Markus Nieberler4; Philipp Blüm²; Nicole Pfarr5; Carolin Mogler; Silvia Thoene ${ }^{6}$; Ramona Secci6; Andreas Bietenbeck ${ }^{6}$; Christoph Straube ${ }^{7}$; Clemens Heiser ${ }^{3}$; Klaus-Dietrich Wolff; Wilko Weichert5; Stephanie Combs7, Florian Bassermann², Jürgen Ruland, Christof Winter ${ }^{1}$

${ }^{1}$ Department of Clinical Chemistry and Pathobiochemistry, Klinikum rechts der Isar, Technische Universität München, Liquid Profiling and Clinical Bioinformatics, München, Germany ${ }^{2}$ Department of Internal Medicine III - Hematooncology, Klinikum rechts der Isar, Technische Universität München, Department of Internal Medicine III - Hematooncology, München, Germany ${ }^{3}$ Department of Otolaryngology, Klinikum rechts der Isar, Technische Universität München, Department of Otolaryngology, München, Germany ${ }^{4}$ Department of Oral and Maxillofacial Surgery, Klinikum rechts der Isar, Technische Universität München, Department of Oral and Maxillofacial Surgery, München, Germany ${ }^{5}$ Department of General Pathology and Pathological Anatomy, Technische Universität München, Department of General Pathology and Pathological Anatomy, München, Germany ${ }^{6}$ Department of Clinical Chemistry and Pathobiochemistry, Klinikum rechts der Isar, Technische Universität München, Department of Clinical Chemistry and Pathobiochemistry, München, Germany ${ }^{7}$ Department of Radiation Oncology, Klinikum rechts der Isar, Technische Universität München, Department of Radiation Oncology, München, Germany

\section{Background and Objective}

For patients diagnosed with head and neck cancer, disease monitoring and detection of tumor recurrence are currently based on clinical examination and imaging. Tumor-derived circulating nucleic acids such as cell-free tumor DNA (ctDNA) can frequently be found in body fluids of cancer patients. Liquid profiling of ctDNA in plasma and in saliva could provide better tumor monitoring and improve the early recurrence detection.Here, we aim to determine to which extent ctDNA and human papillomavirus (HPV) DNA can be detected in plasma and saliva of HNC patients, and which material is more suitable for liquid-based HNC profiling and HPV-related tumor diagnosis. The latter is particularly important as HPV-associated tumors have a better prognosis and might benefit from deescalating therapies.

Methods

In 92 early and late stage HNC patients, blood and saliva samples were prospectively collected after surgery. Samples from all patients were analyzed with digital droplet PCR (ddPCR) assays to detect two TERT promoter hotspot mutations, allowing ctDNA quantification without prior tumor sequencing. In 50 patients with HPV-associated tumor localizations, cell-free DNA (cfDNA) was tested for HPV16 with E7 gene-specific ddPCR primers. In addition, for 9 patients so far, mutations in the primary tumor tissue were identified with panel sequencing ( 45 genes) to quantify ctDNA using individually designed mutation-specific ddPCR assays.

Results

In 33\% (30/92), ctDNA was detected in plasma with mutation-specific ddPCR assays, of which $38 \%$ were early tumor stages (I/II). In p16-positive patients $(n=16)$, cell-free HPV16 DNA was detected in plasma in $39 \%(6 / 16)$, of which $50 \%$ were early tumor stages. In saliva, ctDNA was detected in $52 \%$ (17/33) of samples, and cell-free HPV16 DNA was detected in 15\% (4/27) of samples so far. In patients where both plasma and saliva was available, ctDNA detection rate could be improved from $40 \%$ (plasma only, 16/40) to $58 \%$ (plasma or saliva, $23 / 40$ ). 
In the course of disease, an increase in tumor load could be detected in blood or saliva on average 5.4 months (range 2 weeks to 13 months) earlier than by clinical imaging.

In 63\% (10/16) patients out of the p16-positive cohort, we could not detect HPV16-DNA (E7) in plasma and confirm the clinical HPV-positive status which is based on $\mathrm{p} 16$ expression as a surrogate marker. However, these patients showed an unfavorable clinical course compared to p16-positive HPVpositive patients (30\% died, $79 \%$ had a relapse or progressive disease).

\section{Conclusion}

Liquid profiling with ddPCR-based detection of ctDNA in blood and saliva is a promising tool for disease monitoring and early recurrence detection in head and neck cancer patients. In HPVassociated tumors, cell-free HPV DNA could be a complementary marker for disease monitoring and for identifying a subgroup of $\mathrm{p} 16$-positive tumors that are not driven by HPV infection and hence might benefit from an escalating therapy regime. 
PO-36

Influence of different bead sizes on the binding affinity of anti-factor VIII and von Willebrand
factor as a new diagnostic tool Janine Potreck'; Peter B. Luppa²

${ }^{1}$ Klinikum rechts der Isar der TU München, Institut für Klinische Chemie und Pathobiochemie, München, Germany

${ }^{2}$ Klinikum rechts der Isar der TU München, Klinische Chemie und Pathobiochemie, München, Germany

The motto of this year's DGKL conference is the classic modern motto "form follows function". It is not only the abandonment of ornaments in architecture that is described, but rather a description of an approach to perspective from which new materials and technologies have developed in the workshops of Bauhaus. In particular, interdisciplinary work and higher-level thinking can lead to new findings. Transferred to coagulation, this means that many parameters interact with different influencing factors. Only if a trouble-free expiration of all components takes place, the coagulation can run off and the bleeding is stopped. In hemophilia A, the complex coagulation process is disturbed by a lack of factor VIII. Patients with this disease cannot stop bleeding after injury, as well as spontaneous bleeding, especially in large joints. These circumstances affect the daily lives of patients in a massive way. Similar to classic modern, the focus in diagnostics should be placed on the overall picture. Long time the diagnostic considered only on the interaction of FVIII antibodies with FVIII. But the effect of FVIII, von Willebrand factor (VWF) and anti-FVIII antibodies was neglected. All these parameters are in a direct interaction with each other.

For this reason, we develop a special bead-based fluorescence assay to investigate the competition reaction between anti-FVIII antibody and VWF to FVIII as ligand in patient plasma. In order to visualize both parameter, two monoclonal detection antibodies against VWF and anti-FVIII was additionally added.

With this structure we could already discover the first surprising findings. By using different bead sizes, we have seen that the VWF preferably binds to $7 \mu \mathrm{m}$ beads; the vWF preferably binds to $13,5 \mu \mathrm{m}$ beads. These findings were found both in the control plasma (Bethesda plasma pool) and in patient samples. We cannot yet explain the results to date, but it is thought that the influence of surface curvature, surface functionalization density, and electrostatic interactions play a role. These findings will be examined more closely in the near future. If the binding affinity of the vWF is shown to be influenced by the bead size, then this would be a new approach for the diagnostics of von Willebrand disease (vWD). Instead of using the elaborate multi-parameter analysis, a simpler and faster flowcytometric method can be established. We take advantage of the property that the big vWF is bound disparately on microbeads of different sizes (from 7 to $20 \mu \mathrm{m}$ ). 
PO-37

Development of a laboratory protocol for the detection of MEFV mutations by amplicon
sequencing on a MinION sequencer

Jonas Schmidt'; Folker Wenzel'; Frithjof Blessing²

${ }^{1}$ Hochschule Furtwangen, Medical and Life Sciences, Villingen Schwenningen, Germany

'Laborärzte Singen, Labor, Singen, Germany

Objective: Within the last few years nanopore sequencing has become an important representative of the third-generation sequencing techniques. So far, it is still under development with a continuous increase of accuracy and sequencing throughput. Due to the advantages of fast library preparation and comparably low cost the technique might also be promising for routine genetic diagnostic purpose. Therefore, the objective of our study is to establish a laboratory protocol for the detection of MEFV mutations on the Oxford Nanopore MinION sequencing platform and compare it to Sanger sequencing. Currently, Sanger sequencing is the gold standard for the genetic diagnosis of familial Mediterranean fever which is caused by mutations in the MEFV gene.

Methods: The routine workflow for the diagnosis of familial Mediterranean fever in our laboratory includes DNA isolation from EDTA blood samples by using the QIAamp DNA Blood MiniKit, amplification of the MEFV exons by eight PCR reactions and finally Sanger sequencing of the exons of interest. To test the MinION platform for this approach, the PCR amplicons from one routine sample were pooled after finishing the routine workflow. Library preparation was performed by using the Oxford Nanopore Ligation sequencing kit. The prepared library was loaded on a R9.4.1 flow cell and a six-hour sequencing run was performed. Data analysis was performed on a high-performance workstation by using a custom data analysis pipeline.

Results: By applying the MinION sequencing platform it was possible to sequence all MEFV exons in one run with an extremely high coverage. Therefore, for further analysis the dataset was down sampled. As part of the stepwise routine diagnostic procedure exon 2, 3, 9 and 10 of the chosen sample were already sequenced by Sanger sequencing and seven mutations were found in total. $A$ comparison to Nanopore sequencing revealed that six out of these seven mutations were correctly identified by using the MinION sequencing platform.

Summary: The results indicate that the MinION sequencer combined with an adequate data analysis pipeline in principle is suitable to detect MEFV gene mutations by amplicon sequencing. However, for a final evaluation it is necessary to sequence a larger sample population and compare the results to Sanger sequencing. 


\title{
PO-38
}

The role of telomere attrition and nuclear envelope defects in p21-induced cellular senescence of dermal fibroblasts from pseudoxanthoma elasticum patients

\author{
Janina Tiemann"; Thomas Wagner1; Ricarda Plümers' ${ }^{1}$; Isabel Faust"; Cornelius Knabbe ${ }^{1}$; Doris \\ Hendig ${ }^{1}$ \\ ${ }^{1}$ Herz- und Diabeteszentrum NRW, Universitätsklinik der Ruhr-Universität Bochum, Institut für \\ Laboratoriums- und Transfusionsmedizin, Bad Oeynhausen, Germany
}

Background: Aging is defined as a gradual deterioration of physiological functionality and integrity due to the accumulation of cellular damage. To limit the spreading of cellular damage during aging, a progressively growing amount of cells undergo cellular senescence, leaving the cell cycle for permanent cell cycle arrest. While several stimuli can initially induce cellular senescence, studies e.g. on rare progeria syndromes revealed telomere attrition and defects of the nuclear envelope as two of the most prominent stimuli. Pseudoxanthoma elasticum (PXE) which is mainly caused by mutations in the ATP-binding cassette sub-family $C$ member 6 (ABCC6) gene show some clinical characteristics of the elderly like premature loss of skin elasticity, arteriosclerosis and visual impairment with similarities to macular degeneration. A recent study additionally showed that molecular and cellular biomarkers indicate premature aging processes especially in affected peripheral tissues of PXE patients. Thus, the aim of this study was to further investigate potential premature aging processes associated with cellular senescence and their initiating stimuli in fibroblasts of PXE patients.

Methods: Normal human dermal fibroblasts (NHDF) $(n=3)$ and fibroblasts from PXE patients $(n=3)$ were seeded with a final cell density of $177 \mathrm{cells} / \mathrm{mm}^{2}$. Medium was changed after $24 \mathrm{~h}$. Senescence associated $\beta$-galactosidase activity (SA- $\beta$-gal) was evaluated by fluorescence measurement after additional $72 \mathrm{~h}$ of culturing. Quantitative real-time polymerase chain reaction for analyzing mRNA expression of p21, p53, lamin A, lamin C, progerin and lamin B1 as well as for determination of relative telomere length was conducted $24 \mathrm{~h}$ after initial medium change. Protein expression of lamin $\mathrm{A} / \mathrm{C}$ and lamin B1 were evaluated by immunofluorescence $72 \mathrm{~h}$ after the medium was changed.

Results: SA- $\beta$-gal activity as well as p21 mRNA expression was significantly increased in PXE fibroblasts compared to NHDF. mRNA expression of p53 showed no significant changes. Furthermore, telomeres of PXE fibroblasts were significantly longer compared to NHDF. For lamin A, lamin $\mathrm{C}$ and lamin B1 gene expression we observed a slight decrease in fibroblasts from PXE patients which did not reach statistical significance. mRNA expression of progerin showed a significant increase in PXE fibroblasts. However, we observed no apparent alteration of lamin A/C and lamin B1 protein level or the shape of the nucleus for fibroblasts of PXE patients.

Conclusion: Our data indicate that premature senescence in PXE fibroblasts is probably p21-induced but without upregulation of p53 gene expression and telomere attrition. Further studies are required to evaluate if nuclear architecture defects due to alterations of nuclear envelope components contribute to the induction of cellular senescence in PXE fibroblasts. However, these alterations are unlikely to be the dominant driving force in premature aging of PXE patients. 


\title{
PO-39
}

\section{Evaluation of the effect of heparin, a xylosyltransferase inhibitor, on dermal myofibroblast differentiation}

\author{
Vanessa Schmidt'; Bastian Fischer"; Thanh-Diep Ly'; Doris Hendig'; Cornelius Knabbe ${ }^{1}$; Isabel Faust ${ }^{1}$ \\ ${ }^{1} H e r z-$ und Diabeteszentrum NRW, Universitätsklinik der Ruhr-Universität Bochum, ILTM, Bad \\ Oeynhausen, Germany
}

Background: The xylosyltransferases (XT-I and XT-II) are Golgi-resident enzymes which catalyze the initial rate-limiting step of the proteoglycan (PG) biosynthesis. Thus, both enzymes are substantially involved in the building of the extracellular matrix (ECM). A pathological ECM remodeling with an abnormal rate of PG synthesis is characteristic for a dysregulated wound healing process and can lead to dermal fibrosis. An increased XT-I activity correlates with myofibroblast differentiation - the key step of fibrotic tissue remodeling. Myofibroblast differentiation is induced by TGF $\beta 1$, the main mediator of fibrotic diseases. Based on previous investigations that revealed heparin as a potential intracellular XT-inhibitor, this study evaluates whether heparin has an effect on the wound healing process and myofibroblast differentiation.

Methods: Normal human dermal fibroblasts (NHDF) were cultured under a humidified atmosphere with $5 \% \mathrm{CO} 2$ at $37^{\circ} \mathrm{C}$ under regular conditions (Dulbecco's modified Eagle's medium, $10 \%$ fetal calve serum, $2 \%$ L-glutamine and $1 \%$ antibiotic/antimycotic solution). Cells were seeded at a density of 50 cells/ $\mathrm{mm} 2$ and after a $24 \mathrm{~h}$ serum withdrawal NHDF were treated with heparin $(1 \mathrm{mg} / \mathrm{mL})$ or heparin $(1 \mathrm{mg} / \mathrm{mL})+$ TGF- $\beta 1(5 \mathrm{ng} / \mathrm{mL})$ for additional $72 \mathrm{~h}$ under serum withdrawal. The examination of the myofibroblast differentiation was performed with immunofluorescence staining using an antibody against $\alpha$-smooth muscle actin. Furthermore, we determined the protein expression of collagen type I and fibronectin using western blot analyses to investigate the ECM remodeling. To examine the migration and proliferation ability, NHDF were seeded at a density of 476 cells/ $\mathrm{mm} 2$ and the cell monolayer was scratched after $24 \mathrm{~h}$. NHDF was cultured for another $24 \mathrm{~h}$ under regular conditions with heparin $(1 \mathrm{mg} / \mathrm{mL})$ and the migration was monitored by optical microscopy. Additionally, a WST-1 assay was performed to analyses cell proliferation and viability.

Results and conclusion: Immunofluorescence staining data showed no direct effect of heparin treatment on myofibroblast differentiation. However, western blot analyses indicated an impact of heparin on the ECM remodeling which was detected by a decreased protein expression of collagen type I and fibronectin in the heparin + TGF- $\beta 1$ treated NHDF in contrast to the TGF- $\beta 1$ induced cells. Taking into account the WST-1 assay, the wound healing assay suggested an inhibitory effect of heparin on the migration. In summary, it could be demonstrated that heparin is not only a XT inhibitor but also exerts influence on myofibroblast ECM remodeling. Future studies will be necessary to evaluate the role of heparin during the wound healing process and to investigate possible implications for therapeutic approaches. 


\title{
PO-40
} How to do it right: Tackling the challenge of valid measurements of reactive oxygen species
(ROS) in isolated liver and muscle mitochondria

\author{
Lisa Kappler'; Miriam Hoene;; Paul Grubba; Laxmikanth Kollipara; Albert Sickmann; Andreas \\ Peter'; Cora Weigert'; Rainer Lehmann²
}

${ }^{1}$ University Hospital Tuebingen, Tuebingen, Germany, Clinical Chemistry Central Laboratory, Division of Clinical Chemistry and Pathobiochemistry, Department of Diagnostic Laboratory Medicine, Tübingen, Germany

Universitätsklinikum Tübingen, Institute for Clinical Chemistry and Pathobiochemistry, Department for Diagnostic Laboratory Medicine, Tübingen, Germany

${ }^{3}$ Leibniz-Institut für Analytische Wissenschaften - ISAS - e.V., Protein Dynamics, Dortmund, Germany

Background: Reactive oxygen species (ROS) play a fundamental role in aerobic life and can contribute to many physiological processes as well as the pathogenesis of multiple diseases by causing damages to macromolecules. Mitochondria are a main source for ROS in cells and thus, as key players in metabolism, are involved in different physiological and pathological processes. Therefore, there is the substantial need for a simple, robust and valid technique to measure mitochondrial ROS production in tissues in biomedical research projects.

Objectives and Methods: Amplex Red (AR) is a widely used fluorescent probe for detection of hydrogen peroxide (H2O2). Horseradish peroxidase (HRP) catalyzes hereby the oxidation of AR to the fluorescently detectable resorufin. ROS can be induced by succinate addition causing reverse electron flux or by antimycin A and measured in an Oxygraph-2k. Such an AR assay was applied on muscle mitochondria from mice. However, since AR was reported to be converted to resorufin by a carboxylesterase (CES) without requiring $\mathrm{H} 2 \mathrm{O} 2$, HRP or oxygen, measurements in CES-rich tissues such as liver and kidney can be seriously distorted. To establish a valid ROS method applicable for liver, different approaches were tested in isolated liver mitochondria from mice: on the one hand an inhibitor of CES (phenylmethyl sulfonyl fluoride, PMSF), on the other hand a chelator (diethylenetriaminepentaacetic acid, DTPA), or both. Simultaneously, high-resolution respiration was recorded to investigate possible side-effects of PMSF and DTPA on mitochondrial function.

Results: Proteomics results of isolated mitochondria showed 7 different CES in liver representing $0.4 \%$ of the total protein intensity, but no CES in muscle. Accordingly, ROS production was reproducibly measureable in isolated muscle mitochondria following a widely applied AR protocol inducing ROS by succinate for reverse electron flow and antimycin A without any further adaptions. In liver mitochondria however, measurements of ROS production without or solely DTPA addition, showed a much higher basal level of ROS and then an consistent lowering of the ROS signal over the time course of measurement. No increase in ROS production was detected, even when Antimycin A was added. Addition of PMSF however and the combination of PMSF and DTPA at the beginning of the measurement showed lower basal ROS levels and known ROS producing states (reverse electron flux, Antimycin A) showed increased ROS production. By addition of PMSF alone or PMSF and DTPA the measurement in liver resembled the ROS responses observed for muscle mitochondria. Neither PMSF nor DTPA nor the combination of both showed an effect on mitochondrial function measured as respiration.

Conclusions: We recommend PMSF addition to measurements of ROS in CES-rich tissues such as liver and we would like to argue caution in interpretation of previous data using AR-based methods in such samples. 


\title{
Therapeutisches Drug Monitoring und Pharmakogenetik + Pädiatrische Labormedizin Varia
}

PO-41 / FV-03

\author{
Personalisierte Antibiotika-Therapie bei kritisch kranken Patienten
}

Tony Böhle"; Ulrike Georgi'; Antje Friedrich²; Gudrun Stamminger ${ }^{1}$; Dirk Pohlers ${ }^{1}$

'Zentrum für Diagnostik am Klinikum Chemnitz - Labor Chemnitz, Biochemie/Proteinanalytik, Chemnitz, Germany

Klinikum Chemnitz, Zentralapotheke, Chemnitz, Germany

Zielsetzung: Gerade bei Intensivpatienten mit schweren Infektionen kommt einer rationalen Antibiotika-Therapie eine entscheidende Rolle zu. Untersuchungen belegen, dass insbesondere kritisch kranke Patienten mit veränderter Pharmakokinetik/-dynamik (PK/PD) im Vergleich zur Dosierung nach Standardschema („One dose fits all“) von einer personalisierten Therapie profitieren. Zur Quantifizierung von Ampicillin (AMP), Cefepim (CEF), Cefotaxim (CFO), Cefuroxim (CFU), Ceftazidim (CFT), Linezolid (LIN), Meropenem (MER) und Piperacillin (PIP) soll ein schnelles LC MS/MS-basiertes Verfahren entwickelt und validiert werden. Ferner werden mithilfe von PK/PD Modellen personalisierte Dosisanpassungen erfolgen.

Methoden: Die Antibiotika werden per Dauerinfusion verabreicht, Spiegelbestimmungen wurden $3 \times$ wöchentlich durchgeführt. Die Vollblut-Proben sind bei $28^{\circ} \mathrm{C}$ für mindestens 3 Stunden stabil. Nach der Zentrifugation wird das Serum mittels einfacher Proteinfällung (Acetonitril/Methanol) und Verdünnung (1:10 mit Wasser/Methanol) aufgearbeitet. Alle Messungen wurden an einem Sciex 5500 QTRAP-Massenspektrometer durchgeführt. Die chromatographische Trennung der Analyten erfolgte mit einem Gradienten-Programm (Wasser/Methanol + 0,1\% Ameisensäure; Laufzeit 3,5 Minuten) an einer vollporösen PFP Phase bei $50^{\circ} \mathrm{C}$. Die einzelnen Analyten wurden im MRM Modus anhand von zwei charakteristischen Massenübergängen identifiziert. Für die quantitative Auswertung wurden isotopenmarkierte interne Standards verwendet.

Ergebnisse: Die Validation der LC MS/MS-Methode zeigt gute Intraassay- bzw. InterassayPräzisionen (1,6 8,3\% bzw. 2,3 5,0\%). Matrixeffekte wurden für MER sowie CFO beobachtet und konnten durch die Verwendung von MER d6 bzw. CFO 13C,d3 als interne Standards kompensiert werden. Beim Test auf Interferenzen mit 140 gängigen Medikamenten bzw. deren Metaboliten konnten keine Störungen der Massenspuren gefunden werden. Die Methode zeigt ausreichend große Empfindlichkeiten und lineare Bereiche für die klinische Routine (AMP und CFU 2,5 $180 \mathrm{mg} / \mathrm{l}$; CEF und PIP 2,5 290 mg/l; CFO 160 mg/l; CFT 2,5 360 mg/l; MER 190 mg/l). Abgestimmte Arbeitsabläufe zwischen Probennahme (Station), Messung (Labor) und PK/PD Berechnungen (Apotheke) ermöglichen den Befundrücklauf als personalisierte Dosisempfehlung in weniger als 8 Stunden. Die Erprobung in der klinischen Routine über einen Zeitraum von 6 Monaten zeigt einen gesunkenen Antibiotika-Verbrauch auf den teilnehmenden Stationen.

Zusammenfassung: Die Testphase konnte den Nutzen der TDM-gestützten personalisierten Antibiotika-Therapie belegen. Insbesondere für kritisch kranke Patienten mit veränderter PK/PD sichert sie eine optimale Antibiotika-Wirkung. Die Ausgabe konkreter Dosisempfehlungen statt schwer zu interpretierender Befundberichte führt zu einer hohen Akzeptanz auf ärztlicher Seite. Das Antibiotika-Monitoring soll deshalb auf weitere intensivmedizinische Bereiche ausgedehnt werden. 
PO-42 / PV-06

\section{„Ubiquitous Pharmacogenetics“ (UPGx): das Europäische Projekt zur klinischen Implementierung von pharmakogenetischem Expertenwissen mittels digitaler Lösungen}

Daniela Steinberger ${ }^{123}$

${ }^{1}$ bio.logis Zentrum für Humangenetik ${ }^{2}$ bio.logis Genetic Information Management $\mathrm{GmbH}{ }^{3}$ Institut für Humangenetik, Justus-Liebig Universität Giessen, Medizinische Leitung, Frankfurt am Main, Germany

Bereits seit vielen Dekaden liegen Erkenntnisse zu Assoziationen zwischen häufig vorkommenden genetischen Varianten und deren Auswirkungen auf Arzneimittelwirkungen vor. Darüber hinaus existieren darauf basierende konkrete pharmakologische Empfehlungen, die von internationalen Expertengruppen ausgearbeitet worden sind.

Im Kontrast zu dem vorhandenen exzellent validierten pharmakogenetischen Wissen findet eine klinische Anwendung bisher tatsächlich nahezu nicht statt. In der Präsentation werden die Ursachen sowie Lösungen hierfür, die unter anderem innerhalb eines europäischen Konsortiums in sieben Ländern seit 2017 implementiert wurden, dargestellt. Außerdem werden Zwischenergebnisse der begleitenden klinischen Studie des UPGx-Projektes präsentiert, welche die Untersuchung einer Population von 8.000 Patienten berücksichtigt. 


\title{
PO-43
}

Integrated toxicological screening and confirmation analysis with stable 24/7 availability

\author{
Gry Helene Dihazi"; Marcel Grapp; Tatjana Khromov³; Lutz Binder³; Frank Streit ${ }^{3}$ \\ ${ }^{1}$ UMG-Göttingen, Institut für Klinische Chemiel UMG-Labor, Göttingen, Germany \\ ²Universitätsmedizin Göttingen, Rechtsmedizinisches Labor für forensisch-toxikologische Analytik, \\ Göttingen, Germany \\ 3Universitätsmedizin Göttingen, Institut für Klinische Chemie/ UMG-Labor, Göttingen, Germany
}

Toxins are substances, which even in small doses, may lead to malfunction, damage to health or even death. Prompt toxicological analysis may allow early identification of the toxic agent and provide therapeutic guidance. In clinical laboratories, screening methods using immunoassays based on polyclonal antibodies are commonly applied. However, antibody-based screening methods can cause a significant number of false-positive and false-negative results and, in addition, detected toxins are not quantified. Furthermore, immunological toxicological screening requires a set of 5 to 10 different assays. Nonetheless, comprehensive drug screening and the quantification of relevant substances by chromatography with mass spectrometry is often indispensable. To overcome these shortcomings, our aim was to develop of a liquid chromatography-tandem mass spectrometry (LC-MS/MS) method for targeted screening of a broad spectrum of toxic substances.

From a list of substances most frequently detected in patient samples, which were addressed to the GIZ-Nord Poison Center at the University Medical Center Göttingen in 2014-2018, all relevant compounds were selected. These were integrated into the targeted screening method.

We used an automated sample preparation module (CLAM-2030, Shimadzu) connected to a triplequad LC-MS/MS system (LCMS-8050, Shimadzu). The CLAM-2030 module automatically protein precipitated and filtrated the serum samples before filtrates were automatically transferred into the LCMS/MS. The chromatographic separation of the analytes was performed with a linear gradient on a fused core column at $45^{\circ} \mathrm{C}$. The total run time, including column equilibration, was 7 min. The target compounds were identified by their parent ions with optimized MRM transitions and corresponding retention times. The quantification was done by one-point calibration with trimipramin-D3 as internal standard. The CLAM software was used for automated quantification of identified compounds.

We successfully developed a fast and fully automated LC-MS/MS method for the detection of 152 relevant toxicological compounds. 100 patient serum samples were investigated and results compared with our routine toxicological screening method by QTOF LC-MS. All relevant substances were successfully identified with the novel triple-quad screening method compared to our well-established comprehensive QTOF screening procedure. Repeated measurements of quality control samples revealed less than $20 \%$ inaccuracy for most of the tested substances. Hence, quantification by onepoint calibration is well suited for assessing the severity of an intoxication.

We established a fully automated, robust, quantitative screening method for the detection of more than 150 exogenous substances in serum. This enables targeted toxicological emergency screening in clinical laboratories without skilled personnel. To further refine the method, evaluation and interpretation of the results may be improved and automated. 
PO-44

\section{Mass spectrometry based monitoring of therapeutic antibodies}

Rupert Schreiner'; Dominic Steigerwald ${ }^{2}$; Peter Findeisen ${ }^{2}$; Nina Scheffe ${ }^{2}$

${ }^{1} M V Z$ Labor Dr. Limbach, HPLC, Heidelberg, Germany

${ }^{2}$ MVZ Labor Limbach Heidelberg, Entwicklung, Heidelberg, Germany

Introduction: Monoclonal antibodies (mAbs) are increasingly relevant for the treatment of several diseases including cancer, inflammation, allergies, autoimmune- and infectious diseases. Inadequate $\mathrm{mAb}$ concentration in serum of patients is associated with subsequent loss of response. Hence, therapeutic drug monitoring (TDM) of mAbs has the potential to lead to more effective individualized dosing. However, TDM of mAbs is routinely performed with immunoassays that are affected by antidrug antibodies (ADAs)

Materials and Methods: A mass spectrometry based workflow (LC-MS/MS) was established for the quantification of therapeutic mAbs (Infliximab and Adalimumab) in serum. Several validation parameters including dynamic range, LOD (lower limit of detection) and LOQ (lower limit of quantification), selectivity and reproducibility were evaluated. Results were compared to commercially available immunoassays.

Results: LC-MS based quantification of mAbs from clinical specimens is fit for purpose as test characteristics (LOD, LOQ, linearity and CV) are comparable to those of respective ELISA-kits. Method comparison of IA and MS-assays showed good correlation in absence of ADAs. In contrast, results were significantly different in the presence of ADAs.

Conclusion: Commonly used IAs are only detecting the free portion of mAbs that is reduced in the presence of ADAs. In contrast, the whole amount of mAbs (free and ADA-bound fraction) is detected with MS-based methods. Accordingly, the combination of both methods might facilitate the interpretation of results. Furthermore, MS-based assays improve the standardization of different immunoassays for TDM of Biologicals. 


\section{PO-45}

Accurate assessment of kidney function: a novel high pressure liquid chromatography (HPLC) - tandem mass spectrometry (MS/MS) method for the measurement of iohexol in human serum

Vincent Schweinsberg ${ }^{1}$; Stefan Eckelt ${ }^{2}$; Anna Schulz ${ }^{3}$; Marcel Artt ${ }^{2}$; Marc Schumann ${ }^{2}$; Markus van der Giet $^{3}$; Rudolf Tauber'; Torsten Binscheck-Domaß²; Peggy Kießling ${ }^{1}$

${ }^{1}$ Charité - Universitätsmedizin Berlin; Labor Berlin - Charité Vivantes $\mathrm{GmbH}$, Laboratoriumsmedizin und Toxikologie, Berlin, Germany

'Labor Berlin - Charité Vivantes GmbH, Laboratoriumsmedizin und Toxikologie, Berlin, Germany ${ }^{3}$ Charité - Universitätsmedizin Berlin, Nephrologie, Endokrinologie und Transplantationsmedizin Campus Benjamin Franklin, Berlin, Germany

Objective

Exact measurements of kidney function are essential in a variety of clinical situations, for clinical trials and for other research purposes. The observation of the serum clearance of the hydrophilic $x$-ray contrast agent iohexol has emerged as a feasible and precise technique to measure glomerular filtration rate (GFR) (Krutzen et al. 1984; Gaspari et al. 1995). In contrast to endogenous GFRmarkers, iohexol clearance does not depend on production or metabolisation inside the body nor on renal secretion or reabsorption (Olsson et al. 1983). In addition a very good correlation with the renal clearance of inulin has been shown (Soveri et al. 2014), which is considered to be the gold standard for GFR-measurement. We aimed to develop and validate a novel high pressure liquid chromatography (HPLC) - tandem mass spectrometry (MS/MS) method for the measurement of iohexol in human serum. Relative ion intensities have been used to enhance selectivity and specificity of the assay.

Methods

We applied a simple and quick sample preparation comprising the addition of the internal standard ioversol and protein precipitation with methanol. HPLC was performed with linear gradient elution (mobile phase $A=0,2 \% \mathrm{v} / \mathrm{v}$ formic acid in water; mobile phase $\mathrm{B}=$ methanol). MS/MS-analysis was performed in the Multiple Reaction Monitoring (MRM) mode. The method has been validated in conformity with the requirements for the validation of analytical methods as specified in appendix B to the guidelines for quality assurance in forensic-toxicological analyses published by the Society of Toxicological and Forensic Chemistry (GTFCh). Validation included selectivity and specificity, linearity, imprecision, bias, accuracy, processed sample stability, freeze/thaw stability, long-term stability, limit of detection, lower limit of quantification, recovery, extraction efficiency and matrix effects.

\section{Results}

A novel HPLC-MS/MS method for the quantification of iohexol in human serum has been developed and validated. All validation criteria were met.

Conclusion

The method is now ready for use in clinical routine diagnostics and in research. 


\title{
PO-46
}

\section{Biosensor-based monitoring of TNF antagonists and anti-drug antibodies for patient-tailored therapy of inflammatory autoimmune diseases}

\author{
Melina Grasmeier'; Markus Thaler'; Peter B. Luppa ${ }^{1}$ \\ ${ }^{1}$ Klinikum rechts der Isar der TU München, Institut für klinische Chemie und Pathobiochemie, \\ München, Germany
}

The high prevalence and progressive nature of inflammatory autoimmune diseases like rheumatoid arthritis and inflammatory bowel diease exert a major burden to both patients and healthcare systems. The proinflammatory cytokine tumor necrosis factor (TNF) plays a pivotal role in the pathomechanisms of inflammatory autoimmune diseases and serves as drug target for symptomatic disease management. Even though widely prescribed, treatment of patients with biologic TNF antagonists, e.g. infliximab (IFX), results in broadly diverging outcomes, which can partly be attributed to emergence of anti-drug antibodies (ADAs) that affect therapy success. However, no standardized strategy for therapeutic drug monitoring of TNF antagonists and immunogenicity assessment has been established so far. Due to the drawbacks of other methods, the aim of this thesis was hence to establish SPR-based assays for quantification of both the exemplary TNF antagonist IFX and ADA levels in patient serum.

Two biosensor assays were developed and optimized in terms of sensor chip format, immobilization conditions, regeneration and binding specificity. Furthermore, workflows to correct for non-specific binding and for calibration were established.

Regarding the IFX quantification assay, a ligand cross-linking step included in the immo- bilization protocol was able to enhance the stability of the homotrimeric ligand TNF and hence prolong the sensor lifetime. IFX calibrator signals as well as enhancer antibody (EAb) signals correlated well with nominal IFX concentration. The sensor exhibited good stability over approx. 70 cycles and two days. The IFX quantification test is topically at the verge of being transferred to diluted serum samples, but requires some optimization finetuning regarding regeneration efficiency. The ADA quantification assay was devel- oped taking analogous steps as the IFX monitoring assay and calibration could already be performed in spiked serum using an anti-IFX antibody as calibrator. Extremely mild con- ditions could be used to regenerate the IFX surface on the ADA monitoring sensor, hinting to a long biosensor lifespan. $L O D$ and LOQ were determined as 13 and $15 \mu \mathrm{g} \cdot \mathrm{mL}-1$ of ADA calibrator in diluted serum, respectively and backcalculation of calibrator response to ADA calibrator concentration is expected to be sufficiently reliable for future measurement of authentic serum samples.

The assays developed in this thesis project build a solid base for further optimization fine-tuning and are likely to suffice the quality criteria for measuring patient serum in foreseeable time. Holding potential for sophisticated ADA characterization, SPR-based biosensors employed in routine diagnostics could contribute to a better understanding and management of therapy failure. 


\title{
PO-47
}

\section{LC-MS/MS-Method for the determination of midostaurin - a multitargeted tyrosine kinase inhibitor for the treatment of for acute myeloid leukemia}

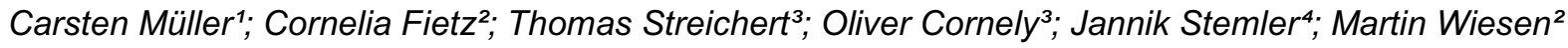 \\ 'Universitätsklinikum Köln, Zentrum für Pharmakologie - TDM, Köln, Germany \\ 'Zentrum für Pharmakologie, Köln, Therapeutisches Drug Monitoring, Köln, Germany \\ ${ }^{3}$ University Hospital of Cologne, Cologne, Germany, Department of Clinical Chemistry, Köln, Germany \\ ${ }^{4}$ University Hospital of Cologne, Cologne, Germany, Department I for Internal Medicine, European \\ Diamond Excellence Centre for Medical Mycology, Köln, Germany
}

Background: Midostaurin (PKC412), a multitargeted tyrosine kinase inhibitor that targets FMS-related tyrosine kinase 3 and KIT, is indicated for the treatment of acute myeloid leukemia (AML) and advanced systemic mastocytosis. In vitro studies demonstrated that midostaurin is predominantly metabolized by cytochrome P450 3A4 (CYP3A4) and that midostaurin inhibits and/or induces the same enzyme. Due to its drug-drug interaction potential and to increase drug safety a reliable and easy to use LC-MS/MS-method for the determination of midostaurin was developed. In addition blood serum samples (midostaurin and posaconazol levels) in AML-patients were collected after achievement of steady-state levels.

Methods: An UHPLC-MS/MS method was developed and validated for determination of midostaurin in serum and plasma matrix. Extraction of serum samples consisted of simple protein precipitation using acetonitrile. A stable isotope labeled analogue for midostaurin was obtained for internal standardization and quantitative analysis. Calibrators and quality controls were prepared in serum matrix of normal individuals. Sample preparation: protein precipitation with acetonitrile and addition of internal standard.

Results: All analytes were eluted within a runtime of 2.5 minutes. Linearity experiments were demonstrated in plasma over a concentration range of midostaurin from $0.01-8.00 \mathrm{mg} / \mathrm{l}$

(R2>0.9995).

Chromatographic separation was achieved using a C18 column $(50 \times 2.1 \mathrm{~mm}, 1.9 \mu \mathrm{m}$ particle size) and an isocratic elution. LC-MS/MS analyses were performed on a triple quadrupole mass spectrometer using positive and negative electrospray ionization in selected reaction monitoring (SRM) mode. Ion transitions monitored for quantitation were $\mathrm{m} / \mathrm{z} 571.22$--> 348.05 for midostaurin and 576.20 --> 348.10 for stable isotope labeled analogue.

Inter- and intra-day precisions (CV, \%) varied between 3.6 and 8.2 and inter- and intra-day accuracy values ranged from $-8.7-6.5 \%$. The lower limit of detection and quantification were: 0.01 and 0.02 for midastaurin.

Conclusion: Although the level of recommendation of TDM of midostaurin in patients with acute myeloid leukemia is not yet established, the present LC-MS/MS-method can serve as an effective tool for detection of midostaurin in this vulnerable subpopulation of hemato-oncological patients and may impact on patients outcome with regard to drug-drug interaction potential of comedication. 


\title{
PO-48
}

\section{Investigations of clinical samples show that direct measurements of DOACs is advisable under certain circumstances}

\author{
Joachim Kuhn"; Andreas Hammerschmidt1; Isabel Faust'; Doris Hendig"; Cornelius Knabbe ${ }^{1}$; Ingvild \\ Birschmann ${ }^{1}$
}

${ }^{1}$ Herz- und Diabeteszentrum NRW, Universitätsklinik der Ruhr-Universität Bochum, Institut für Laboratoriums- und Transfusionsmedizin, Bad Oeynhausen, Germany

Objective: Measurement of direct oral anticoagulants (DOACs) concentration in patient blood is essential in special clinical circumstances.

Methods: A total of 120 clinical samples (45 samples from patients which were treated with apixaban, 27 samples from patients which were treated with dabigatran, 8 samples from patients which were treated with edoxaban, and 40 samples from patients which were treated with rivaroxaban) were examined. Direct measurements of DOACs were performed using our selective and sensitive automated online solid-phase extraction ultra-performance liquid chromatography-tandem mass spectrometry (SPE-UPLC-MS/MS) assay. These findings were compared with the results of commercially available coagulation assays for these DOACs samples.

Results: No apixaban could be detected in 7 of the 45 samples from patients which were treated with apixaban by direct measurement using the SPE-UPLC-MS/MS assay. In addition, the apixaban concentration in further 4 samples was very low $(<1 \mu \mathrm{g} / \mathrm{L})$ and one sample contained rivaroxaban instead of apixaban. Furthermore, by direct measurement of the DOACs, no dabigatran could be detected in 5 of the 27 patient samples which were treated with dabigatran, no edoxaban could be detected in 2 of the 8 patient samples which were treated with edoxaban, and no rivaroxaban could be detected in 12 of the 40 patient samples which were treated with rivaroxaban. In several cases, the mass spectrometric results could not be confirmed by coagulation-based assays and vice versa, although good correlations between direct and functional measurements of apixaban, dabigatran, and rivaroxaban were found when these extreme cases were excluded. No regression analysis between SPE-UPLC-MS/MS assay and coagulation assay was performed for edoxaban measurements because of the small sample size. However, it can be calculated from the measured values that the concentration measured by mass spectrometry was generally lower than measured by the functional assay.

Conclusion: In summary, direct measurement of apixaban, dabigatran, edoxaban, and rivaroxaban in patient samples is advisable if coagulation-based assay resulted in a doubtful outcome and mass spectrometric measurement of the DOACs is here the method of choice. 


\title{
PO-49 / PV-04
}

\section{Newborn screening for severe combined immune deficiencies - a pilot study prior the nationwide implementation}

\author{
Theresa Winter1; Maria Gizewska²; Kasia Durda3; Jeanette Klein ${ }^{4}$; Oliver Blankenstein ${ }^{4}$; Mariusz \\ Ołtarzewski'; Małgorzata Pac ; Matthias Nauck ${ }^{7}$ \\ 'Universitätsklinikum Greifswald, Neugeborenen Screening Labor, Greifswald, Germany \\ ${ }^{2}$ Pomorski Uniwersytet Medyczny w Szczecinie, Newborn Screening Laboratory, Szczecin, Poland \\ ${ }^{3}$ Samodzielny Publiczny Szpital Kliniczny Nr 1 PUM, Newborn Screening Laboratory, Szczecin, \\ Poland \\ ${ }^{4}$ Charité Campus Virchow-Klinikum, Neugeborenen Screening Labor, Berlin, Germany \\ 5Institute for Mother and Child, Newborn Screening Laboratory, Warsaw, Poland \\ 'Institute "Pomnik - Centrum Zdrowia Dziecka", IPCZD, Warsaw, Poland \\ ${ }^{7}$ Institut für Klinische Chemie und Laboratoriumsmedizin, Neugeborenen Screening Labor, Greifswald, \\ Germany
}

Introduction:

Severe combined immune deficiencies (SCID) are a very heterogenic group of genetic disorders. They are characterized by severe deficiencies of essential immune defense $T$ and/or B-cells which can be cured by stem cell transplantation. The incidence is approximately 1:50.000, but realistic numbers for Germany are not known. The federal joint committee (G-BA) decided to implement SCID in the regular newborn screening (NBS) panel in 2019, which leads to several new organizational challenges for screening laboratories and birth clinics.

Within the EU-funded Interreg Va project "RareScreen" (INT10), experiences with SCID NBS are obtained, which shall be shared prior the nationwide implementation.

Material and Methods:

Since October 2018, all newborns in the Interreg Va funding area (Western-Pomerania, NorthBrandenburg (Germany) and West Pomerania Province (Poland)) can participate in an extended NBS which includes SCID. Out of the dried blood cards, which are uses for the regular NBS, TRECs (T-cellreceptor excision circles) and KREC (Kappa-deleting recombination excision circles) are being measured in a quantitative RT-PCR. The number of TRECs and KRECs correlates with newly developed $\mathrm{T}$ - and $\mathrm{B}$-cells and allows information about $\mathrm{T}$ - and $\mathrm{B}$-cell deficiencies. The project covers more than planned for the nationwide SCID NBS implementation, in which a TREC- only approach was decided by the G-BA. The participating screening laboratories Berlin and Greifswald currently send their sample to the screening laboratory in Szczecin, where the ImmunoIVD SCID kit (ImmunoIVD, Sweden) is used.

Results:

Since October 2018, samples of over 17.000 newborns were investigated in terms of SCID. Reasons for repeated analysis of the first sample were insufficient actin amplification, which runs simultaneously as an internal quality control or TREC and KREC copy numbers below the cutoff. The percentage of repetitions out of the first screening card had a range of 0.9-2.6\% between the three participating screening laboratories. The range of requested second screening card ranged from $0.05-0.5 \%$. So far two positive SCID case can be reported.

Discussion and conclusion:

Since October 2018, the participating screening laboratories gain valuable experiences about the SCID NBS. Methodically, the SCID NBS is a challenge for the screening laboratories, since the quantitative RT-PCR is so far not used for the regular NBS. But the first months of SCID screening showed, that the used kit/method works reliable. The range of initially conspicuous SCID results of the 
first sample card is quite wide. Reasons are either pre-analytic errors, such as false sample taking (e.g. use of EDTA blood) or the incorrect punching of the card.

After a positive SCID screening, processing steps such as confirmation diagnosis and, if necessary, initiation of a therapy have be clarified until the nationwide introduction starts to ensure a stable treatment- and tracking procedure. 


\title{
PO-50
}

\section{Neugeborenen Screening auf familiäre Hypercholesterinämie - eine internationale Machbarkeitsstudie}

\author{
Theresa Winter ${ }^{1}$; Elisabeth Steinhagen-Thiessen²; Matthias Nauck ${ }^{3}$ \\ ${ }^{1}$ Universitätsklinikum Greifswald, Neugeborenen Screening Labor, Greifswald, Germany \\ ${ }^{2}$ Charite - Universitätsmedizin Berlin, Medizinische Klinikfür Endokrinologie und Stoffwechsel, Berlin, \\ Germany \\ ${ }^{3}$ Institut für Klinische Chemie und Laboratoriumsmedizin, Neugeborenen Screening Labor, Greifswald, \\ Germany
}

\section{Einleitung:}

Die familiäre Hypercholesterinämie $(\mathrm{FH})$ ist eine angeborene Störung des Lipidstoffwechsels, die durch eine ausgeprägte Erhöhung des LDL-Cholesterins (Low Density Lipoprotein) im Plasma charakterisiert ist. Die Folge ist eine frühzeitige Manifestation der koronaren Herzkrankheit. Es wird eine Häufigkeit einer heterozygoten FH von 1:500 angenommen und somit zählt die FH zu den häufigsten genetischen Störungen. Meist wird eine FH in nur ca. 15 \% der Fälle diagnostiziert. Ansätze für ein universales $\mathrm{FH}$-Screening gibt es in verschiedenen Ländern ab einem Alter von 5 Jahren sowie über eine Pilotstudie aus Nabelschnurblut wurde berichtet. Wir stellen hier eine Machbarkeitsstudie eines FH-Screenings im Rahmen des etablierten Neugeborenenscreenings (NGS) vor.

\section{Material und Methoden}

Seit Oktober 2018 werden Kinder in Mecklenburg-Vorpommern, Brandenburg und Westpommern (Polen) im Rahmen des EU-geförderten Interreg Va Studie „RareScreen“ auf FH untersucht. Dafür wird aus der regulären Trockenblutkarte des NGS das Gesamtcholesterin mittels LC-MS/MS bestimmt. Anhand der gemessenen Cholesterinkonzentrationen konnte bereits ein cut-off errechnet werden. Auf dieser Basis können nun Familien kontaktiert werden, deren Neugeborene Cholesterinwerte über der 99,9 Perzentile aufweisen, um die Machbarkeit des NGS und eines möglichen Kaskadenscreenings zur Erkennung von betroffenen Familienmitgliedern zu überprüfen.

\section{Ergebnisse}

Seit Oktober 2018 wurden mehr als 12.000 Neugeborenen auf FH untersucht. Die Gesamtcholesterinwerte aller Neugeborenen zeigen sich normalverteilt. Es zeigt sich kein statistisch signifikanter Zusammenhang zwischen dem Alter, Geburtsgewicht und Ernährungsstatus (Muttermilch, parenterale Ernährung oder Babynahrung) der Kinder. Zwischen Frühgeborenen $(<32$. SSW) und Reifgeborenen zeigt sich ein signifikanter Unterschied in der

Gesamtcholesterinkonzentration, mit höheren Werten bei den Reifgeborenen. Aktuell werden Kinder mit Cholesterinwerten über der 99,9 Perzentile identifiziert und die entsprechenden Familien kontaktiert. Die ersten Einbestellungen in spezielle Lipidambulanzen sind initiiert.

\section{Diskussion oder Schlussfolgerung:}

Bemerkenswert ist, dass weder das Alter, noch das Geburtsgewicht und der Ernährungsstatus der Neugeborenen mit dem gemessenen Cholesterinwerten korreliert sind. Das unterstreicht die starke Rolle des genetischen Hintergrunds der Cholesterinwerte, die aber noch belegt werden muss. Der Rücklauf aus den Einbestellungen der Eltern mit ihren Kindern in spezielle Lipidambulanzen wird Informationen liefern, wie robust und verlässlich diese Früherkennungsstrategie ist. Anders als im regulären NGS, könnten mit diesem Ansatz im ersten Schritt primär die Eltern, die mit großer Wahrscheinlichkeit nicht von ihrer FH wissen, profitieren und erst nachgeordnet die Neugeborenen selbst. Aktuelle Fortschritte im Bereich der lipidsenkenden Therapie könnten in naher Zukunft auch Kindern mit $\mathrm{FH}$ eine frühzeitige Therapie ermöglichen. 


\title{
Inflammation, Infektion und Autoimmunerkrankungen -1
}

\section{PO51 / PV-09}

\section{Thrombomodulin regulates platelet and extracellular vesicle mediated sterile inflammation in the placenta}

\author{
Shrey Kohli'; Satish Ranjan²; DHEERENDRA GUPTA²; Shruthi Krishnan²; Berend Isermann² \\ 'Universität Leipzig, Institut für Laboratoriumsmedizin, Klinische Chemie und Molekulare Diagnostik, \\ Leipzig, Germany \\ ${ }^{2}$ Otto-von-Guericke-Universität Magdeburg Medizinische Fakultät, Instituts für Klinische Chemie und \\ Pathobiochemie, Magdeburg, Germany
}

Objectives: Thrombomodulin (TM) deficiency causes tissue-factor initiated thrombin generation, maternal platelet-activation and embryonic lethality in mice. However, the mechanism remains poorly understood. We have recently shown that procoagulant extracellular vesicles (EVs) activate maternal platelets, which results in purinergic receptor mediated inflammasome activation in the trophoblast and subsequently preeclampsia (PE). We therefore investigated whether this EV-mediated thromboinflammatory pathway in placenta is controlled by thrombomodulin and whether this mechanism contributes to the lethality of TM-null embryos.

Methods: Procoagulant EVs were injected into C57/BI6 pregnant mice. Placental-TM expression was studied using immunoblotting and immunofluorescence. EV-injected pregnant mice were treated with soluble TM (solulin) to establish causality. Genetic and pharmaceutical inflammasome inhibition was conducted to establish mechanistic relevance in TM-null placenta. Differentiated mouse trophoblast stem cells were treated with EV or IL-1 $\beta$ to study the inflammasome associated loss of TM. Trophoblast proliferation and cell death was studied using Ki-67 and TUNEL staining respectively. Translational relevance was corroborated by analysis of human PE placentae and trophoblast cells.

Results: EV caused PE and accumulation of activated platelets within the placenta. EV treatment resulted in a reduced TM expression and trophoblast proliferation in mouse placenta in-vivo and EVtreated mouse or human trophoblast cells in-vitro. Trophoblast cell death was increased. Furthermore, TM expression was negatively correlated with IL-1 $\beta$ expression and platelet counts in human PE placentae. However, genetic (NLRP3-/-) or pharmaceutical (Anakinra, Apyrase) inflammasome inhibition did not rescue the TM-null embryos from lethality. Treatment of mouse or human trophoblast cells with exogenous IL-1 $\beta$ resulted in reduced TM expression indicating that loss of TM is the consequence, and not cause of inflammasome activation. Further, Solulin treatment and overexpression of TM in the placenta ameliorated the PE-like phenotype in mice and prevented thrombo-inflammation and restored trophoblast proliferation.

Summary: These results demonstrate that EV-mediated platelet-activation, thromboinflammation and PE are associated with loss of placental TM and support a pathophysiological association of thrombophilia associated pregnancy complications and sterile inflammation. While loss of TM seems to be a consequence rather than cause of inflammasome activation, treatment with solulin efficiently prevented the EV-induced thromboinflammatory response and PE-like phenotype, presumably by restricting coagulation and platelet activation. 


\title{
PO-52 / PV-12
}

\section{Identification of a urate crystal recognition molecule in human body fluids by mass spectrometry}

\author{
Konstantin Neumann'; Andreas Pich ${ }^{2}$; Korbinian Brand ${ }^{1}$; Christèle Combes ${ }^{3}$; Anika Alberts ${ }^{1}$ \\ ${ }^{1}$ Medizinische Hochschule Hannover, Institut für Klinische Chemie - Zentrallabor, Hannover, Germany \\ ${ }^{2}$ Medizinische Hochschule Hannover, Institute of Toxicology, Core Unit Proteomics, Hannover, \\ Germany \\ ${ }^{3}$ Institut National Polytechnique de Toulouse, CIRIMAT, Toulouse, France
}

The deposition of crystals within joints leads to strong inflammatory responses. The crystallization of uric acid (monosodium urate, MSU) in the joints leads to gouty arthritis, while calcium pyrophosphate dihydrate crystallization leads to pseudogout.

It is believed that the inflammation in both conditions is caused by the complement system, direct activation of immune cells and activation of the NLRP3 inflammasome within immune cells. However, it remains rather unclear, if and how these crystals are recognized by the complement system and immune cells. We have previously identified a transmembrane receptor (Clec12A) that specifically recognizes MSU. It inhibits certain inflammatory responses induced by MSU, so activating receptors remain ill defined.

Given that binding of complement or other opsonins to crystals may be sufficient for indirect recognition of crystals by immune cells, we set out to identify all soluble MSU binding molecules in human body fluids.

We incubated MSU crystals with different human sera, washed the crystals extensively, and eluted the bound proteins. The eluted proteins were identified by high-resolution mass spectrometry. We were able to identify all previously known MSU-binding proteins, including apolipoproteins and most proteins of the complement cascade.

In addition, we found a novel MSU-binding protein that binds with such high specificity that it can be specifically depleted from human serum/plasma by addition of MSU crystals. This protein was also found in synovial fluid of patients with arthritis, from which it can be depleted by addition of MSU even more efficient than from serum/plasma.

Intriguingly this protein, while showing binding to several other types of crystalline structures, does neither bind to monoclinic nor triclinic calcium pyrophosphate dihydrate crystals.

Identification of this novel MSU recognition molecule may shed light on the general mechanisms of immune recognition of crystalline structures. 


\section{PO-53 / PV-07}

\section{Tissue factor pathway inhibitor primes monocytes for antiphospholipid antibody-induced thrombosis}

Nadine Müller-Calleja'; Karl J. Lackner'; Wolfram Ruf ${ }^{2}$

'Universitätsmedizin Mainz, Institut für klinische Chemie \& Laboratoriumsmedizin, Mainz, Germany ${ }^{2}$ Universitätsmedizin Mainz, Centrum für Thrombose \& Hämostase (CTH), Mainz, Germany

\section{Aim/Background}

Antiphospholipid antibodies (aPL) with complex lipid and/or protein reactivities cause complementdependent thrombosis and pregnancy complications. While cross-reactivities with coagulation regulatory proteins contribute to the risk for developing thrombosis in patients with antiphospholipid syndrome, the majority of pathogenic aPL retain reactivity with membrane lipid components and rapidly induce reactive oxygen species-dependent proinflammatory signaling and tissue factor (TF) procoagulant activation. In this study, we focused on the early cellular events by which aPL influence the TF pathway finally leading to pathogenic signaling and thrombosis amplification.

\section{Methods}

We studied human and mouse monocytic cell activation by aPL by single stage clotting assay, induction of genes and ROS, and molecule tracking by confocal microscopy. In vivo thrombus formation was measured using the vena cava flow restriction model.

\section{Results}

Here, we show that lipid-reactive aPL activate a common species-conserved TF signaling pathway: aPL dissociate an inhibited TF coagulation initiation complex on the cell surface of monocytes, thereby liberating factor $X a$ for thrombin generation and protease activated receptor $1 / 2$ heterodimer signaling. In addition to proteolytic signaling, aPL promote complement- and protein disulfide isomerasedependent TF-integrin $\beta 1$ trafficking that translocates aPL and NADPH oxidase to the endosome. TF pathway inhibitor (TFPI) synthesized by monocytes is required for TF inhibition; and disabling TFPI prevents aPL signaling, indicating a paradoxical prothrombotic role for TFPI. Myeloid-cell specific TFPI inactivation has no effect on models of arterial or venous thrombus development, but remarkably prevents experimental aPL-induced thrombosis in mice.

\section{Conclusion}

Lipid-reactive antiphospholipid antibodies (aPL) trigger coagulation and inflammatory signaling by dissociating an TFPI inhibited TF-FVIla-FXa cell surface complex. Thereby, myeloid cell-expressed TFPI specifically supports aPL-induced thrombosis. 


\section{PO-54 / PV-08}

\section{CD248 regulates ER stress in diabetic nephropathy}

Shruthi Krishnan"; Ronald Biemann'; Sophie Mathieu1; Jayakumar Manoharan'; Akshay Malhotra2; Michael Naumann'; Berend Isermann ${ }^{1}$

Otto-von-Guericke-Universität Magdeburg, Institute for Clinical Chemistry and Pathobiochemistry, Magdeburg, Germany

Otto-von-Guericke-Universität Magdeburg, Institute of Experimental Internal Medicine, Magdeburg, Germany

OBJECTIVE- Diabetic nephropathy (DN) is a major cause of end-stage renal disease and a growing health challenge. Endoplasmic reticulum (ER) stress is associated with DN, but the mechanisms that compromise adaptive ER signaling during hyperglycemia still remain resolved. We focused on CD248, a type I transmembrane glycoprotein expressed by pericytes, such as glomerular mesangial cells and tubulointerstitial fibroblasts whose expression correlates with renal fibrosis and inflammation in chronic kidney disease. The major goal of our study is to investigate the role of CD248 in regulating ER stress and sterile inflammation in DN.

METHOD- C57BI/6 (WT) mice and CD248-/- mice were used to study the impact of CD248 on DN progression in vivo. Diabetes was induced by intraperitoneal administration of streptozotocin (STZ) and samples were obtained after 26 weeks. Albuminuria (UACR), PAS staining and WT-1 immunostaining of kidney sections were analysed. To evaluate mechanistic insights, the consequences of CD248 knockdown or overexpression were investigated in vitro by immunoblotting and co-immunoprecipitation approaches.

RESULTS- Renal CD248 expression increased after 26 weeks in diabetic mice versus nondiabetic controls. CD248 ablation reduced diabetes induced albuminuria, podocyte loss and mesangial matrix accumulation. In vitro, shRNA mediated CD248 knockdown in renal pericytes attenuated high glucose induced ER stress (lower levels of CHOP, ATF4, cATF6 $\alpha$ ), inflammasome activation (lower levels of NLRP3, cleaved IL-1 $\beta$ ) and mTORC1 activation (lower levels of p-Raptor and p-p70S6Kinase). Vice versa, overexpression of CD248 amplifies these glucose induced responses. Mechanistically, the chaperone HSP90 interacts with the transmembrane receptor CD248 and may thus transduce extracellular glucose-dependent stress signals to the ER via IRE-1 $\alpha$.

SUMMARY- Pericyte specific CD248 regulates hyperglycemia induced ER stress and inflammatory response. HSP90 appears to serve as the cytosolic mediator linking the receptor CD248 with ER membrane proteins such as IRE1 $\alpha$. Our findings identify CD248 as a potential target for therapeutic interventions of DN. 


\title{
PO-55 / PV-10
}

\section{Role of evolutionary conserved cold shock protein YB-1 in unidirectional glomerular tubular communication in diabetic kidney disease}

\author{
Rajiv Rana1; Ahmed Elwakiel2; Jayakumar Manoharan²; Jonathan Lindquist ${ }^{3}$; Sabine Brandt ${ }^{3}$; Silke \\ Zimmermann'; Peter Mertens'; Berend Isermann² \\ 'Institut für Klinische Chemie und Pathobiochemie, Magdeburg, Germany \\ ${ }^{2}$ Otto-von-Guericke-Universität Magdeburg Medizinische Fakultät, Institut für Klinische Chemie und \\ Pathobiochemie, Magdeburg, Germany \\ ${ }^{3}$ Otto-von-Guericke-Universität Magdeburg, Medizinische Fakultät, Institutes für Molekulare und \\ Klinische Immunologie, Magdeburg, Germany
}

OBJECTIVE- Diabetic nephropathy (dNP) is a major cause of end stage renal disease, characterized by podocyte dysfunction, accumulation of extracellular matrix in glomerular and the peritubular space, and tubular dilation. A glomerular-tubular crosstalk as a driving pathomechanism in diabetic nephropathy has been suspected, but the paracrine signals remain largely unknown. As (1) diabetic nephropathy is closely associated with inflammation, (2) the cold shock protein YB-1 is known to modulate inflammation, and (3) YB-1 modulates renal disease, we speculated that YB-1 contributes to diabetic nephropathy, potentially mediating glomerular-tubular interaction. The major goal of our study is to investigate the role of podocyte secreted YB-1 in regulating inflammation in tubular cells in DN.

METHOD- We used YB1LoxP mice without Cre-expression (WT-mice) or with podocyte (glomerular epithelial cells) specific YB-1 knockout mice (YB1 $\square$ Pod mice) and induced persistent hyperglycaemia using streptozotocin (DM-mice), while control received sodium citrate injections (control-mice). As endpoints we analysed albuminuria (primary endpoint) as well as morphological and molecular changes associated with dNP.

RESULTS- DM-YB1 $\square$ Pod mice displayed higher albuminuria and aggravated tubular dilation compared to DM-WT mice. Surprisingly, the mesangial area was reduced in DM-YB1 $\square$ Pod mice compared to DM-WT mice. These findings implied glomerular protection but enhanced tubular damage in DM-YB1 $\square$ Pod mice. The enhanced tubular damage was associated with an increased expression of inflammasome activation markers (NLRP3, IL-1 $\beta$ ) in whole kidney extracts in DMYB1 $\square$ Pod mice. We therefore hypothesized a protective paracrine effect of extracellular YB-1 secreted from podocyte on tubular cells under hyperglycaemic condition. Mechanistically, pre-treatment of tubular cells with recombinant YB-1 following LPS stimulation inhibits TLR4 mediated NF-KB and inflammasome activation in vitro, indicating that recombinant YB-1 negatively modulates Toll-like receptor-4 (TLR4) pro-inflammatory signalling renal in tubular cells.

SUMMARY- Our data suggest that extracellular YB-1 secreted from podocytes regulates NF-kB activation via TLR4 receptor in tubular cells. These findings indicate that modified or recombinant YB-1 can be used as a potential therapeutic approach for CKD and that YB-1 in urine may be a potential marker for renal damage. 


\section{PO-56}

The role of integrins for signaling of serine protease activated protein C in T-cells

DHEERENDRA GUPTA ${ }^{1 ;}$ Satish Ranjan"; Ahmed Elwakiel'; Shuthi Krishnan"; Andreas Müller2; Shrey Kohl'1; Berend Isermann ${ }^{1}$

${ }^{1}$ Medical Faculty, Otto-von-Guericke University, Magdeburg, Institute for Clinical Chemistry \& Pathobiochemistry, Magdeburg, Germany

${ }^{2}$ Medical Faculty, Otto-von-Guericke University, Magdeburg, Institute for Molecular and Clinical Immunology, Magdeburg, Germany

Introduction: The serine protease activated protein C (aPC) is an anticoagulant, which additionally conveys intracellular signals via protease activated receptors (PARs). The function of aPC in innate immunity through both its anticoagulant and signaling properties is established. Using a mice model of GvHD, we recently established a function of aPC and one of its receptors (PAR3) in adaptive immunity. However, PAR3 is not signaling competent by itself and requires co-receptors. Interestingly, aPC has been shown to interact with integrins through its RGD-domain. Integrin activation and signaling has been shown to be a key mechanism regulating T-cell activation, adhesion and migration. Here we test the hypothesis whether aPC regulates T-cell activation, adhesion and migration via integrins on T-cells.

Methods: GvHD was induced in aPChigh, RGE-aPChigh, and C57BL/6 (WT, Control) mice by transplanting $1 \times 106$ T-cells with $5 \times 106$ bone marrow cells from BALB/c mice. Survival, clinical scores and cell death in intestinal cells of gastrointestinal tract were used as parameters to study disease severity. Murine DTH model was induced by TNCB followed by syngeneic transplantation of aPC preincubated T-cells from challenged mice (BALB/C). In vivo migration was studied in these mice by twophoton microscopy. T-cell activation and adhesion were studied in vitro. The effect of aPC on integrin signaling was studied using immunoblotting and FACS.

Results: Mice with endogenous high levels of aPC (aPChigh) were protected from GvHD in comparison to WT mice. However, mice expressing a mutant aPC lacking the integrin binding site (RGE-aPChigh) were not protected, indicating that interaction of aPC with integrins is required for its immunomodulatory function in GvHD. Surprisingly, we observed in vitro that aPC additionally regulates the activation of non-RGD integrin like LFA-1. Using phospho-tyrosine antibody we observed global phosphorylation alterations. Pre-incubation of T-cells with aPC reduced their activation, adhesion to ICAM1 and fibronectin and phosphorylation of Ezrin (a cytoskeletal protein) along with a decline of active-Rap1 (a key modulator of LFA-1 activation).

Conclusion: Collectively, these data suggest that aPC modulates the immune response of T-cells via two independent signaling pathways. aPC interacts with PAR3, which apparently reduces global phosphorylation and thus modulates inside-out signaling of integrins. In addition, aPC apparently binds via its RGD domain to RGD-binding integrins, affecting T-cell function via outside-in signaling. In future studies we will dissect the molecular pathways and evaluate the pathophysiological relevance of these findings in more detail. 
PO-57

\title{
Established engine Protein Array Platform enables identification new biomarkers for differentiation of autoimmune diseases
}

\author{
Mathias Puschmann"; Jörg-M. Hollidt ${ }^{2}$ \\ ${ }^{1}$ engine gmbh, Hennigsdorf, Germany \\ ${ }^{2}$ engine gmbh, Geschäftsführung, Hennigsdorf, Germany
}

Objective: Due to the unregulated immune response in autoimmune diseases, a variety of serum antibodies are produced in patients. This is a challenge in biomarker identification, as specificity of the potential marker is essential in diagnostic practice. Therefore, securing the potential marker against differentiation to other autoimmune diseases should already be considered and tested at the very beginning. This is possible by screening sera from patients with different autoimmune diseases on engine Protein Arrays.

engine hEXselect and UniPEx Protein Arrays display up to 23,806 E.coli-expressed proteins and peptides of human cDNA libraries generated from fetal brain, lung and T-cell. The Protein Arrays are produced by contact spotting of the E.coli clones, which express the proteins directly on the PDVFmembrane-based array. They do not require tedious freeze shipment/storage and are as easy to use as a western blot. Further, as E. coli-expressed proteins mostly lack post-translational modification, specific enzyme-based modifications, such as citrullination, can be introduced on engine Protein Arrays, when required. Even more, custom-made subarrays of selected proteins can be generated and allow cost-effective screening of larger cohorts. The protein subarrays used here are such a subset of 827 proteins. These proteins were selected from previous screenings of engine hEXselect Protein Arrays with sera pools from patients with systemic autoimmune diseases.

Preliminary Data: The sera of 17 patients and 2 healthy donors were diluted 1:1000 in blocking buffer and incubated on a subarray over night at room temperature under shaking. Subsequently, detection was performed with an AP-conjugated anti-human-lgG secondary antibody and Attophos as substrate. Fluorescent signals were classified as positive, when the duplicates of the clones showed comparable fluorescence intensities above background. 22 different antigens were detected positive in sera of the self-reported healthy donors. In patients with thyroid diseases (Hashimoto thyroiditis and Graves' disease, n=4) 99 different antigens were detected. 193 antigens were detected in Patients with RA $(n=3)$ and Sjögren syndrome $(n=1)$. In the sera of 3 multimorbid patients 141 antigens could be identified. In all 6 patients with systemic lupus erythematosus (SLE) a panel of 7 different proteins could be determined, with each SLE patient reacting with up to 4 of the 7 markers. Of the 11 patients with other autoimmunity diseases, one with Graves' Disease reacted to 2 of the markers and one with $\mathrm{RA}$ reacted to 3 markers of this panel.

Conclusion: Each patient has an individual antibody profile, which is strongly influenced by the disease. Antibodies are therefore an almost infinite source of biomarkers. The comparison of antibody profiles of different diseases is essential to find specific markers. engine Protein Arrays offer an optimal platform for biomarker screening due to their large protein repertoire. 


\title{
PO-58
}

Phosphorylation of Ubiquitin at serin-20 and its impact on the coordination of cellular functions in human cells.

\author{
Martin Christmann'; Rolf Bikker'; Korbinian Brand ${ }^{1}$
}

Medizinische Hochschule Hannover, Institut für Klinische Chemie, Hannover, Germany

Ubiquitin is a small protein involved in numerous cellular functions within all kingdoms of life. We currently identified a phosphorylation site at serin-20 of ubiquitin, which is so far, functionally uncharacterized. We further observed a significant reduction of phosphorylation at serin-20 within ubiquitin after long-term TNF exposition. Tumor necrosis factor (TNF) is a master cytokine orchestrating inflammatory response in humans. The coordination of TNF signaling requires various posttranslational modifications. Disturbed TNF signal perception is a hallmark of numerous human diseases, such as SIRS and Sepsis or chronic inflammatory maladies like rheumatoid arthritis or inflammatory bowel disease as well as malignant processes. Here we report the recent status of our investigations of the ubiquitin phosphorylation site at serin-20 in human cells in general, as well as after distinct stimuli, such as TNF or PMA in monocyte cells. Thus a more comprehensive picture of ubiquitin regulation will be obtained. Furthermore, the potential of Ubiquitin serin-20 as a marker and/or target for diagnosis and prognosis of inflammatory diseases as well as malignant processes will be explored. 


\title{
PO-59 / PV-11
}

\section{Uremia-induced microglial activation promotes IL-1beta dependent neuronal dysfunction independent of the canonical caspase-1- dependent NLRP 3-inflammasome}

\author{
Silke Zimmermann"; Klarina Waldmann²; Berend Isermann²; Akash Mathew² \\ 1Otto-von-Guericke-Universität Magdeburg, Medizinische Fakultät, Institut für Klinische Chemie und \\ Pathobiochemie, Magdeburg, Germany \\ ${ }^{2}$ Universitätsklinikum Magdeburg, Institut für Klinische Chemie und Pathobiochemie, IKCP, 39120 \\ Magdeburg, Germany
}

Background:

Chronic kidney disease (CKD) causes cognitive impairment and dementia. Alteration such as uremic toxins affect neuronal function and survival. The molecular mechanisms remain unknown. We observed high pro-IL-1 $\beta$ expression and IL-1 $\beta$ generation in uremia-induced microglia activation despite a lack of canonical caspase-1-NLPR3-inflammasome activation. We hypothesize that CKD and associated uremic toxins cause microglial activation via caspase-8, which contributes to inflammasome activation within the CNS and thus to neuronal impairment.

Aim: We aim to determine the relevance of inflammasome activation in uremia-induced cognitive dysfunction in different celltypes and the mechanisms of IL-1 $\beta$ activation in microglia.

Methods and materials:

We used thallium-autometallography (TI-AMG) to monitor cellular K+-metabolism. CKD in mice was induced by $5 / 6$ nephrectomy (5/6 NX). To study cell-activation in the CNS in vivo we conducted twophoton intravital microscopy. We used double transgenic mice expressing EGFP under the control of the CX3C chemokine receptor 1 (CXC3R1) promoter (labeling microglia). NLRP3 and Casp-1 knockout mice (NLRP3-/-, Casp-1-/-, C57BL/6 background) were used to determine the mechanistic relevance of inflammasome activation. We generated new mouse models required for the experiments. We obtained mice carrying NIrp3A350VneoR, causing an inducible point-mutation in exon3 and thereby leading to a cell-specific hyperfunction of NLRP3. We injected the IL1b $\beta$-receptorInhibitor Anakinra in mice. We performed immunfluorescence on human and murine microglia and neurons.

Results:

Mice with CKD revealed altered K+-metabolism in the CNS and increased expression of caspase-1 and IL-1 $\beta$ in whole brain lysates. Two-photon microscopy revealed activation, but not significantly increased cell death, of microglia and neurons in mice with CKD. Analyses of wild type C57BL/6 and NX NLRP3-/- mice six weeks after inducing CKD revealed a comparable number of thallium positive activated microglia, indicating that the uremia-induced reactive shift is a process independent of NLRP3 inflammasome activation.

However, in 5/6 NX NLRP3-/- mice as well as in CASP-1-/- the decrease of neuronal thallium uptake was abolished, indicating that the NLRP3 inflammasome is indeed involved in CKD-induced K+dyshomeostasis of neurons, hence neuronal dysfunction.

We further show the activation of microglial cells in CKD leads to secretion of II1 $\beta$. We identify an alternate pathway of IL1 $\beta$ cleavage via Caspase8-RIPK1/3 in microglia.

Conclusion:

These data suggest that uremia induces non-necroptotic Caspase 8 activation via RIPK1/3 in microglia cells, hence leading to cleavage of II1 $\beta$ in microglia. Hence, we propose that activation of 
microglia independently of the canonical inflammasome is pivotal in the pathophysiology of CKDinduced impairment of brain function. These results may lay ground for new diagnostic approaches. 


\section{Inflammation, Infektion und Autoimmunerkrankungen -2}

\section{PO-60}

\section{Clinical evaluation of a turbidimetric assay for quantification of fecal Calprotectin}

Christian Niederberger'; Marie-Eve Überschlag²; Chris Moore ${ }^{3}$; Alanna Grob; Peter Kupchak

${ }^{1} B U ̈ H L M A N N$ Laboratories AG, International Affairs, Schönenbuch, Switzerland

${ }^{2} B U ̈ H L M A N N$ Laboratories AG, Validation Team, Schönenbuch, Switzerland

${ }^{3} B U ̈ H L M A N N$ Laboratories AG, Marketing, Schönenbuch, Switzerland

${ }^{4} B D C$, Clinical Studies, Amherst NH, United States

${ }^{5}$ Statistical Consultancy, Statistics, Toronto, Canada

Background: Fecal calprotectin ( $\mathrm{fCAL}$ ) is a non-invasive biomarker used to differentiate between inflammatory bowel disease (IBD) and irritable bowel syndrome (IBS). We sought to evaluate the performance characteristics of the BÜHLMANN fCAL® turbo in differentiating between IBD and IBS in adults and children as well as explore test performance in a variety of other gastrointestinal (GI) disorders.

Methods: Turbidimetric measurement of clinically determined patient samples from a multi-center prospective observational case-control study, previously measured with the BÜHLMANN fCAL ELISA, was conducted in 416 subjects. IBD, IBS, Patients with other GI disorders and normal subjects were included if strict inclusion criteria were met. The samples were processed with the original manual extraction method as well as with the prefilled CALEX Cap extraction device.

Results: Both methods showed very distinct calprotectin level characteristics for the different subject groups, by determination of median fCAL values seen in IBD patients compared to IBS patients and normal subjects. In differentiating IBD from IBS, the BÜHLMANN fCAL turbo (PETIA) shows sensitivity $91.3 \%$ specificity $74.8 \%$ at a cut-off $80 \mu \mathrm{g} / \mathrm{g}$ and sensitivity $81.1 \%$ and specificity $85.4 \%$ at a cut-off $>160 \mu \mathrm{g} / \mathrm{g}$ with an AuROC of 0.923. CALEX extract showed good agreement.

Conclusions: The BÜHLMANN fCAL turbo demonstrates excellent performance characteristics in discriminating between IBD and IBS and a high correlation to the ELISA reference standard method. The application of an automated technology such as the BÜHLMANN fCAL turbo will improve the laboratory capability through reduced turn-around times and improved workflow management for random access and high throughput sample processing. CALEX Cap further streamlines the laboratory workflow. 
PO-61

Multiple Sclerosis related sensitivity of FLC-Kappa evaluated with a new hyperbolic reference
range

Malte Hannich'; Hansotto Reiber²; Astrid Petersmann ${ }^{3}$; Matthias Nauck ; Alexander Dressel4; Marie Süße ${ }^{5}$

'Uni Medizin Greifswald, Institut für Klinische Chemie und Laboratoriumsmedizin, Greifswald, Germany

Universität Göttingen, CSF and Complexity Studies, Göttingen, Germany

${ }^{3}$ Universitätsmedizin Greifswald, Institut für klinische Chemie und Laboratoriumsmedizin, Greifswald, Germany

${ }^{4}$ Carl-Thieme-Klinikum Cottbus, Neurologie, Cottbus, Germany

${ }^{5}$ Universitätsmedizin Greifswald, Klinik und Polyklinik für Neurologie, Greifswald, Germany

Introduction: Free light chains kappa (FLC-k) in cerebrospinal fluid (CSF) are a promising biomarker for diagnosis of clinically isolated syndrome (CIS) or multiple sclerosis (MS). Recently several comparative studies suggested the use of the FLC-k-Index as the appropriate instrument for the processing of the single values of CSF and serum, though a consensus about the reference interval is missing. In 2019 Reiber et al. provided a hyperbolic reference interval respecting the nonlinear relation between the albumin- and the FLC-K-quotient of CSF and serum. The aim of this study was to evaluate the diagnostic performance of FLC-k in patients with CIS and MS using the hyperbolic function by Reiber et al. instead of the commonly used FLC-k Index. We also assessed if the new approch could help differenciate between MS and CIS.

Methods: FLC-k were determined in 109 samples of patients with MS and CIS by nephelometry using the Siemens N Latex FLC kappa Kit on the Siemens BN Prospec. The FLC-k CSF/serum-quotient was plotted against the CSF/serum albumin-quotient. The hyperbolic function established by Reiber et al. was applied as a reference interval. Comparison concerning the diagnostic sensitivity for MS and CIS with the different FLC-k Indices was conducted. FLC-k values were additionaly evaluated for clinical correlations (influence of disease modifiying drugs, gadolinium (Gd) enhancement in MRI, conversion to MS).

Results: For our clinically defined patient group only measurement results above the newly established reference interval of the FLC-k were observed, reaching a sensitivity in this patient cohort of $100 \%$ to detect intrathecal inflammation in patients with CIS and MS in comparison with $99 \%$ and $96 \%$ using different FLC-k Indices (3.61 and 6.6). No significant differences in FLC-kloc concentrations could be detected regarding DMD taking, Gd enhancement in MRI or conversion to MS in CIS patients.

Conclusion: The evaluation of FLC-k values in hyperbolic quotient diagrams based on the molecular flux/ CSF flow theory in patients with CIS and MS showes a superior sensitivity to other analytical methods like the linear FLC-k Index. We could not confirm an earlier described diagnostic benefit using the hyperbolic reference interval for FLC-k to distinguish between MS and CIS. 
PO-62

\title{
The differential LPS response of the liver in the context of diet-induced obesity
}

\author{
Paul Heller'; Anke Witt'; Bettina Gercken'; Iryna Pyrina1; Kyoung-Jin Chung1; Peter Mirtschink'; \\ Triantafyllos Chavakis ${ }^{2}$ \\ ${ }^{1}$ Universitätsklinikum Carl Gustav Carus, TU Dresden, Institut für Klinische Chemie und \\ Laboratoriumsmedizin, Dresden, Germany \\ Universitätsklinikum Carl Gustav Carus Dresden, TU Dresden, Institut für Klinische Chemie und \\ Laboratoriumsmedizin, Dresden, Germany
}

\section{Aim}

Recent clinical studies reported an improved survival rate of overweight and obese patients in sepsis (Pepper et al., $2019^{*}$ ); however, the underlying mechanisms are unknown. In this context, we investigated the differential metabolic response in the liver of high fat diet (HFD) and normal diet (ND) fed mice followed by lipopolysaccharide (LPS)-induced acute systemic inflammation.

Methods

C57BL/6N mice were subjected or not to diet-induced obesity (i.e. fed a HFD or ND) for 8 weeks followed by acute LPS-induced systemic inflammation. PBS injected mice served as control.

\section{Results and Conclusion}

Mice fed a HFD for 8 weeks displayed a significant increase in bodyweight and basal blood glucose level, as compared to the ND-fed control group. Despite increased blood glucose levels at baseline, obese mice displayed a LPS-induced hypoglycemia, which was not different from lean mice. Immunohistochemical analysis of the liver revealed a complete depletion of hepatic glycogen stores at $24 \mathrm{~h}$ after LPS injection in both obese and lean mice. Consistently, decreased mRNA expression of enzymes involved in glycogen synthesis and glycogenolysis was observed in livers of LPS-treated mice, which is also seen in obese mice that received PBS suggesting a decreased hepatic glycogen metabolism by both obesity and acute inflammation. Moreover, we observed an upregulation of various glycolytic enzymes in the liver in response to LPS and/or HFD treatment at both mRNA and protein level.

By immunofluorescence staining of the liver for CD13, a canalicular surface marker, we could detect an increased branching of the bile canalicular network upon LPS-treatment, as an indicator of cholestasis. In accordance with these observations, the gene expression of the main bile acid synthesis enzyme Cyp7A1 in the liver was significantly lower in LPS treated mice. The cholestatic phenotype was more pronounced in the HFD-fed mice compared to ND-fed controls.

Taken together, preliminary results point to alterations in glucose and bile metabolism in obese mice, which are further affected by LPS injection. We currently characterize the cholestatic and hypoglycemic phenotype in diet-induced obesity and acute systemic inflammation using molecular biology and mass-spectrometry based metabolomic analyses.

\footnotetext{
* Pepper DJ, Demirkale CY, Sun J, et al.Does Obesity Protect Against Death in Sepsis? A Retrospective Cohort Study of 55,038 Adult Patients. Crit Care Med. 2019;47(5):643-650.
} 


\section{PO-63}

\section{Associations of YKL-40 with bone quality in the general adult population}

Jörn Steinke'; Stefanie Samietz²; Nele Friedrich'; Stefan Weiß33; Reiner Biffar; Matthias Nauck'; Uwe Völker5; Henri Wallaschofski'; Maik Pietzner'; Anke Hannemann ${ }^{4}$

${ }^{1}$ Universitätsmedizin Greifswald, Institut für Klinische Chemie und Laboratoriumsmedizin, Greifswald, Germany

${ }^{2}$ Universitätsmedizin Greifswald, Poliklinik für zahnärztliche Prothetik, Alterszahnheilkunde und medizinische Werkstoffkunde, Greifswald, Germany

${ }^{3}$ Universitätsmedizin Greifswald, Institut für Klinische Chemie und Laboratoriumsmedizin, Greifswald,

Germany

${ }^{4}$ Universitätsmedizin Greifswald, Interfakultäres Institut für Genetik und Funktionelle Genomforschung, Greifswald, Germany

${ }^{5}$ Universitätsmedizin Greifswald, Poliklinik für zahnärztliche Prothetik, Alterszahnheilkunde und medizinische Werkstoffkunde, Greifswald, Germany

Background: Chitinase-3-like protein 1 (CHI3L1), also known as YKL-40, is a new proinflammatory biomarker, that might play a role in tissue remodeling, immune response and bone resorption. We evaluated the associations of the YKL-40 plasma concentration with quantitative ultrasound (QUS)based parameters at the heel and two bone turnover markers (BTM): intact amino-terminal propeptide of type I procollagen (PINP) and carboxy-terminal telopeptide of type I collagen (CTX) in adult men and women from the general population. Additionally, we assessed whether a selected singlenucleotide polymorphism (SNP) at the CHI3L1 gene locus is associated with the QUS-based parameters and the BTMs.

Methods: Data were obtained from the first and second follow-up of the population-based Study of Health in Pomerania (SHIP-1 and SHIP-2) and the baseline SHIP-Trend cohort. Associations between the YKL-40 plasma concentration and the QUS-based parameters, the BTM concentrations and the genetic variation at $\mathrm{CHI} 3 \mathrm{LI}$ (rs4950928) were evaluated in a subsample of $420 \mathrm{SHIP}$-Trend participants. Associations between rs4950928 and the BTM concentrations were assessed in 7190 SHIP-1 and SHIP-Trend participants and associations between rs4950928 and the QUS-based parameters were assessed in 5777 SHIP-2 and SHIP-Trend participants.

Results: Sex-specific linear regression models adjusted for age, waist circumference, smoking, 25hydroxy vitamin $\mathrm{D}$, intake of analgesics, platelets and position on plate revealed statistically significant inverse associations between YKL-40 and all QUS-based parameters as well as positive associations with the BTMs in women only. The rs 4950928 polymorphism was associated with YKL-40 in men and women but neither with the QUS-based parameters nor the BTMs after correction for multiple testing.

Conclusion: Plasma YKL-40 concentrations are associated with QUS-based parameters as well as BTM concentrations in women but these associations are probably not causal. 
PO-64

\title{
Identification of A Novel Xylosyltransferase-I Regulatory Mechanism by miRNA-145 in Dermal Fibroblasts Derived from Systemic Sclerosis Patients
}

\author{
Thanh-Diep Ly'; Ricarda Plümers ${ }^{2}$; Bastian Fischer; ${ }^{3}$ Vanessa Schmidt; Doris Hendig ${ }^{3}$; Joachim \\ Kuhn ${ }^{3}$; Cornelius Knabbe ${ }^{3}$; Isabel Faust ${ }^{3}$ \\ ${ }^{1}$ Herz- und Diabeteszentrum Nordrhein-Westfalen, Universitätsklink der Ruhr-Universität Bochum, \\ Institut für Laboratoriums- und Transfusionsmedizin, Bad Oeynhausen, Germany \\ ${ }^{2}$ Herz- und Diabeteszentrum Nordrhein-Westfalen, Universitatsklink der Ruhr-Universitat Bochum, \\ Institut fur Laboratoriums- und Transfusionsmedizin, Bad Oeynhausen, Germany \\ ${ }^{3}$ Herz- und Diabeteszentrum Nordrhein-Westfalen, Universitätsklinik der Ruhr-Universität Bochum, \\ Institut für Laboratoriums- und Transfusionsmedizin, Bad Oeynhausen, Germany
}

Introduction: Fibrosis, a major health problem worldwide, is characterized by the accumulation of extracellular matrix in response to physical and inflammatory insults. Patients suffering from Systemic Sclerosis (SSc), the prototype of chronic fibrotic skin diseases, show an excess deposition of collagenous fibers and proteoglycans (PGs) as well as an increase in profibrotic growth factors in the dermis. Serum analysis from SSc patients revealed an increase in enzyme activity of xylosyltransferase (XT), which is the initial enzyme in PG biosynthesis. In humans, two distinct XT isoforms, $\mathrm{XT}$-I and XT-II, exist whereas only XT-I is associated with fibrotic changes and provides a reliable myofibroblast marker. However, the molecular mechanism by which fibrosis is induced has not been fully understood and also involves epigenetic elements like microRNAs (miRNAs). The aim of our study is to gain new insights into the molecular mechanism underlying fibrosis in SSc. We hypothesize that one triggering event, by which cytokines of the TGF $\beta$ superfamily contribute to dermal fibrosis, is mediated by rapid upregulation of XYLT1 expression and XT activity in dermal fibroblasts leading to the former detected increase in serum XT activity and PG content. Methods: We investigated the regulation of XT-I in human dermal fibroblasts (NHDF) and SSc fibroblasts (SScF) and the implicated regulatory pathways using an appropriate cell culture model. The expression of miRNA-145, XYLT1 mRNA and associated genes was assessed by quantitative real time PCR. The determination of $\mathrm{XT}$ activity was performed by radiochemical enzyme assay. The involvement of miRNA-145 and transcription factors in TGF $\beta$ mediated fibrotic response in NHDF was investigated using miRNA mimics, inhibitors and siRNA-mediated gene silencing. The evaluation of miRNA-145 binding to the XYLT1 3'UTR was done by luciferase reporter assay. Results: TGF $\beta 1$ is a potent inducer of XYLT1 expression in NHDF und SScF. Basal expression of miRNA-145 is downregulated in SScF, contributing to the observed expression increase of its target gene Krüppel-Like Factor 4 (KLF4). In comparison to untreated NHDF cells TGF $\beta 1$ treatment resulted in significant increase of miRNA-145 level. The inducible effect of TGF $\beta$ on XT-I expression was enhanced after overexpression of miRNA-145 in NHDF. Direct XYLT1 3'UTR targeting by miRNA-145 could be experimentally excluded indicating an indirect effect of this miRNA on XT-I regulation. In-silico target analysis revealed KLF4 and Mothers Against Decapentaplegic Homolog 3 (SMAD3) to be direct miRNA-145 targets. Performing of siRNA mediated silencing of either KLF4 or SMAD3 confirmed the inhibitory effects of both transcription factors on XYLT1 expression in NHDF. Conclusion: Our study shows a new connection of dysregulated miRNA-145 pathway and immediate induction of XT-I expression by TGF $\beta$ signaling via downregulation of KLF4 and SMAD3, improving the understanding of fibrotic remodeling in SSc. 


\section{PO-65}

Different immunoassays for the detection of anti-cardiolipin and anti- $\beta 2$-glycoprotein
antibodies: Importance of verifying the manufacturer's cutoff values

Ivana Markovic'; Gry Helene Dihazi'; Frank Streit'; Abass Eidizadeh'; Lutz Binder ${ }^{1}$

${ }^{1}$ UMGL Göttingen, Klinische Chemie, Göttingen, Germany

Background and aim

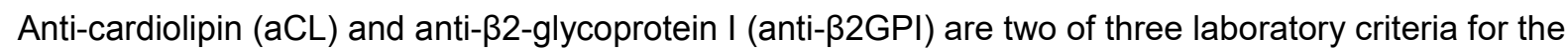
diagnosis of anti-phospholipid syndrome (APS). All immunoassays detecting anti-phospholipid antibodies (aPL), as well as lupus anti-coagulance assays, show methodological shortcomings, which affect their sensitivity and specificity. To improve the quantitative detection of these antibodies and correctly classify patients as positive for the anti-phospholipid syndrome (APS), we evaluated the analytical and clinical performance of $\operatorname{lgG}$ and $\operatorname{lgM}$ aCL antibodies as well as $\lg G$ and $\operatorname{lgM}$ anti- $\beta 2 \mathrm{GPI}$ antibodies. We additionally discussed and re-evaluated the upper reference limit (99th percentile) claimed by the Sidney Criteria in a healthy population compared to the manufacturer's cutoff values of various commercial methods.

Methods

We collected serum samples from 41 healthy controls, 7 patients with antiphospholipid syndrome (APS) diagnosed according to the Sydney criteria, 10 patients with different autoimmune diseases and 6 seronegative rheumatologic patients. $\operatorname{lgM} / \mathrm{lgG}$ aCL and $\operatorname{lgM} / \mathrm{lgG}$ anti- $32 \mathrm{GPI}$ antibodies were determined by CLIA Bio-Flash (Werfen, Barcelona, Spain) and by EliA Phadia (Laboratory Systems, Thermo Fischer, Germany). In addition, IgM/lgG aCL were determined using a conventional ELISA from Orgentec, Germany.

\section{Results}

We found a poor quantitative correlation between the assays. However, there was an acceptable qualitative accordance (fourfold table), based on individual antibodies and patient categories. After revision of the respective cutoffs under consideration of healthy controls, qualitative method comparison revealed excellent analytical accordance, strong correlation with APS-related clinical symptoms and thus good clinical performance APS diagnosis.

\section{Conclusions}

According to guidelines, all laboratories are strongly recommended to verify the manufacturers' cutoff values. The investigation of healthy controls is a useful additional tool to establish or validate cutoffs with respect to a clinically correct APS diagnose.

\section{Keywords}

anti- $\beta 2$ glycoprotein I (anti- $\beta 2 \mathrm{GPI}$ ) antibodies; anti-cardiolipin ( $\mathrm{aCL}$ ) antibodies; anti-phospholipid antibodies (aPL); anti-phospholipid syndrome (APS); chemiluminescence immunoassay (CLIA); enzyme-linked immunosorbent assay (ELISA). 


\title{
PO-66
}

\section{LC-MS/MS method for measuring inosin-5'-monophosphate dehydrogenase activity in patients treated with mycophenolic acid}

\author{
Martin Wiesen"; Gero Weißbarth²; Cornelia Fietz'; Thomas Streichert³; Rasmus Ehren; Lutz Weber"; \\ Carsten Müller ${ }^{1}$
}

\author{
${ }^{1}$ University of Cologne, Faculty of Medicine and University Hospital Cologne, Germany, Center of \\ Pharmacology, Therapeutic Drug Monitoring, Cologne, Germany \\ 2University of Cologne, Faculty of Medicine and University Hospital Cologne, Germany, Center of \\ Pharmacology, Therapeutic Drug Monitoring and Paediatric Nephrology, Children's and Adolescents' \\ Hospital, Cologne, Germany \\ ${ }^{3}$ University of Cologne, Faculty of Medicine and University Hospital Cologne, Germany, Institute for \\ Clinical Chemistry, Cologne, Germany \\ ${ }^{4}$ University of Cologne, Faculty of Medicine and University Hospital Cologne, Germany, Paediatric \\ Nephrology, Children's and Adolescents' Hospital, Cologne, Germany
}

BACKGROUND: Mycophenolic acid (MPA), the active moiety of mycophenolate mofetil (MMF), is widely used in transplantation medicine and as glucocorticoid-sparing agent in various inflammatory diseases. MPA pharmacokinetics is complex and highly differs between patients. Therapeutic Drug Monitoring (TDM) of MPA by means of serum or plasma concentration measurement is a standard approach to avoid underexposure of MPA and toxicity. Because inosine-5'-monophosphate dehydrogenase (IMPDH), the target enzyme of MPA, shows high inter-individual variability of its basal activity, assessment of IMPDH activity in addition to pharmacokinetic monitoring is a promising strategy to optimize MPA pharmacotherapy.

METHODS: A liquid chromatography-tandem mass spectrometry (LC-MS/MS) method was developed to calculate IMPDH activity in peripheral blood mononuclear cells (PBMC) from lithium-heparinized blood. Following incubation of PBMC lysates with inosine 5'-monophosphate (IMP) as substrate and $\beta$ nicotinamide adenine dinucleotide (NAD+) as co-factor, produced xanthosine-5'-monophosphate (XMP) and intracellular adenosine-5'-monophosphate (AMP) were measured. IMPDH activity was expressed as enzymatic production of XMP per time normalized to AMP concentration. Stable isotopelabeled analogues of XMP and AMP were used as internal standards for quantitative analysis. Method evaluation was performed with a focus on linearity and specificity of XMP formation over time and inhibition of IMPDH activity by increasing MPA concentrations using blood samples obtained from healthy volunteers $(n=10)$.

RESULTS: Linear XMP and AMP quantification could be demonstrated over the concentration ranges of $0.25-80 \mu \mathrm{M}$ and $4-80 \mu \mathrm{M}(\mathrm{R} 2>0.99)$, respectively. Inter- and intra-day assay precisions and accuracies remained well within the acceptance criterion of $\pm 15 \%$. Matrix effects were compensated by co-eluting internal standards, and calculated lower limits of quantification were $0.16 \mu \mathrm{M}$ for XMP and $0.78 \mu \mathrm{M}$ for AMP. A specific and linear XMP production (R2 > 0.99) could be demonstrated in PBMC lysates within an incubation time of 150 minutes. Incubation of PBMC lysates with MPA in clinically relevant doses showed a typical sigmoidal dose-response relationship. The calculated IC50 was $16.7 \mathrm{nM}$.

CONCLUSION: In this study, an improved LC-MS/MS method was established that enables precise XMP and AMP quantification and calculation of IMPDH activity. Clinical studies are needed to investigate whether a combined pharmacokinetic/pharmacodynamic TDM approach helps to optimize immunosuppressive therapy with MPA. 


\title{
PO-67
}

\section{Biomarker for the prediction of responsiveness to treatment with anti-Tumour Necrosis factor alpha (anti-TNFa)}

\author{
Mathias Puschmann'; Jörg-Michael Hollidt ${ }^{2}$
}

${ }^{1}$ engine gmbh, Hennigsdorf, Germany

'in.vent Diagnostica GmbH, Geschäftsführung, Hennigsdorf, Germany

Objective: $30-40 \%$ of all RA patients are TNFa Non-responder therefore it is necessary to identify these patients before treatment. New biomarkers were searched using protein arrays. Based on these markers, a companion diagnostic as ELISA will improve the treatment decision in RA patients.

Biomarker Screening: The commercially available hEXselect Protein Arrays (engine GmbH / \#1003) were used to identify novel biomarkers for prediction of responsiveness to treatment with anti-TNFa. Sera from patients with different responsiveness were screened for IgA-autoantibodies. The engine hEXselect Protein Arrays based on 23,806 E.coli-expressed proteins and peptides of a human fetal brain cDNA-library. The diluted sera and after that anti-human-IgA-AP antibody were incubated. Fluorescent signals were classified as positive, when intensities of clone duplicates above background. Five intracellular proteins were identified as biomarkers: RAB11B, COG4, FDFT1, PPP2R1A and KPNB1.

ELISA development: The antigens expressed in E.coli and His-Tag-affinity-purified under denaturating conditions. With these high pure proteins five independent 96 well colorimetric sandwich ELISAs have been developed at in.vent Diagnostica $\mathrm{GmbH}$. The ready-to-use ELISAs based on antigen coated $8 \times 12$ strip plate. Due to the 1:50 dilution of the sample, only $2 \mu \mathrm{l}$ serum is required and the procedure takes less than $3 \mathrm{~h}$. The ELISAs were optimized to a working range of $0-300$ units $/ \mathrm{mL}$ with a lower limit of detection of $<5$ units $/ \mathrm{mL}$, the linear calibration curves enables accurate measurement. During the reaction step, the bounded patient antibodies react specifically with anti-human-IgA-HRP. The precision analysis of this commercially available ELISAs was performed based on the CLSI EP05-A3 guidelines using a single-site approach with 16 samples. Over all five ELISAs could be achieved an intra-assay $\mathrm{CV}$ of $<10 \%$ and an inter-assay $\mathrm{CV}$ of $<15 \%$.

Independent validation: In a cooperation between DRDx GmbH and Charité Berlin, the biomarkers were tested for their clinical utility for the identification of Non-responders. 203 patients with RA and 164 self-reported healthy were tested. In the healthy samples, a specificity of $97-99 \%$ could be achieved over all biomarkers. With a sensitivity of $80.5 \%$ TNFa Non-responder could be distinguished from Responder. With a specificity of $93.8 \%$ Non-responder could be detected as Non-responder.

Conclusions: The identification of strong predictive markers is important for optimization of the therapy regime and so for patients. Protein arrays are a reliable screening platform to identify new biomarkers. Our stable and useable research-use-only ELISAs for the determination of autoimmune markers helps to evaluate the clinical relevance. The clinical data, generated by third parties, indicate the high potential, but further clinical studies are necessary to evaluate these preliminary clinical findings. 


\title{
PO-68
}

\section{Macrophage-derived extracellular vesicles mediate macrophage polarization and contain bioactive lipids}

\author{
Mitja Leonard Heinemann'; Silvana Müller; Madlen Reinicke; Uta Ceglarek'; Joachim Thiery ${ }^{1}$ \\ ${ }^{1}$ Universitätsklinikum Leipzig, Institut für Laboratoriumsmedizin, Klinische Chemie und Molekulare \\ Diagnostik (ILM), Leipzig, Germany
}

Background: Extracellular vesicles (EV) facilitate intercellular communication by transporting a complex package of proteins, lipids, and nucleic acids from cell to cell. Their exact composition depends on type and state of the originating cell. Although EVs are known to also contain a variety of bioactive lipids, the function of these EV lipids is largely unknown to date. Current literature attributes EVs a particularly important role in inflammation and immune response. However, interpretation of functional analyses is impeded by the large heterogeneity of the currently applied EV isolation methods, some with questionable purity, others with questionable functional integrity of the isolated EVs. In order to overcome these limitations, we recently developed a gentle, filtration-based EV isolation method for downstream functional EV analysis.

Objectives: Our aim was to study functional effects of EVs isolated by sequential filtration using the example of EV-mediated macrophage polarization. Furthermore, we aimed at proving the presence of bioactive lipids in the isolated EVs.

Methods: THP-I monocytes were differentiated into macrophages by PMA and polarized into M1 (IFNy/LPS) or M2 (IL-4) state. EVs derived of polarized (M1/M2) and unpolarized (M0) macrophages were purified by sequential filtration and added to unpolarized macrophage cultures. After 24 hours of culturing, cells were harvested and expression of M1/M2 markers was assessed by quantitative realtime PCR. Bioactive lipid composition was assessed by means of LC-MS/MS.

Results: EVs derived of M1 macrophages led to a significant upregulation of M1 markers (TNF-a, IL1B, IL-6, CXCL-10) in target cells, compared to M0-derived EVs. EVs derived of M2 macrophages led to a significant upregulation of M2 markers (CD23, CD209) in target cells, compared to M0-derived EVs. By LC-MS/MS analysis, we could detect multiple eicosanoids and polyunsaturated fatty acids (e.g. TxB2, TxB3, 12-HETE, DHA, ARA) in EVs derived of M0-, M1- and M2-macrophages.

Conclusion: In summary, we could show that EVs derived of polarized macrophages and isolated by sequential filtration can induce polarization in target macrophages. We could prove the presence of bioactive lipids in all EV preparations, which may hint towards a functional involvement of EV lipids in the immune response. 


\title{
Leitlinien und diagnostische Pfade + Metobolom/Lipidom/Proteom/Glykom = Omics
}

PO-69

\author{
Die Kommission für Labordiagnostik in der Diabetologie (KLD) stellt sich vor
}

Astrid Petersmann'; Guido Freckmann ${ }^{2}$; Arnold von Eckardstein ${ }^{3}$; Liselotte Lennartz; Peter B. Luppa ${ }^{5}$; Christoph Niederau ${ }^{6}$; Nele Friedrich ${ }^{1}$; Henri Wallaschofski $i^{7}$ Ulrich Müller; Andrea Tytko'; Dirk MüllerWieland $^{10}$; Erwin Schleicher ${ }^{11}$; Rüdiger Landgraf ${ }^{12}$; Matthias Nauck'; Lutz Heinemann ${ }^{13}$

'Universitätsmedizin Greifswald, Institut für Klinische Chemie und Laboratoriumsmedizin, Greifswald, Germany

${ }^{2}$ Forschungs- und Entwicklungsgesellschaft $\mathrm{mbH}$ an der Universität UIm, Institut für DiabetesTechnologie, Ulm, Germany

${ }^{3}$ Universitätsspital Zürich, Institut für Klinische Chemie, Zürich, Switzerland

${ }^{4}$ Abbott Diagnostics, Abbott Laboratories, Wiesbaden, Germany

${ }^{5}$ Technische Universität München, Institut für Klinische Chemie und Pathobiochemie, München, Germany

${ }^{6}$ Dr. Niederau und Kollegen, Medizinisches Versorgungszentrum für Labormedizin, Mikrobiologie und Infektionsepidemiologie, Dortmund, Germany

${ }^{7}$ Praxis für Endokrinologie, Praxis für Endokrinologie, Erfurt, Germany

${ }^{8}$ Universitätsklinikum Jena, Klinik für Innere Medizin III, Jena, Germany

${ }^{9}$ Diabetespraxis Northeim, Diabetespraxis Northeim, Northeim, Germany

${ }^{10}$ RWTH Aachen, Medizinische Klinik I, Aachen, Germany

${ }^{11 U n i v e r s i t a ̈ t s k l i n i k u m ~ T u ̈ b i n g e n, ~ Z e n t r a l l a b o r, ~ I n n e r e ~ M e d i z i n ~ I V, ~ T u ̈ b i n g e n, ~ G e r m a n y ~}$

${ }^{12}$ Deutsche Diabetes Stiftung (DDS), Deutsche Diabetes Stiftung (DDS), München, Germany

'Universitätsmedizin Greifswald, Institut für Klinische Chemie und Laboratoriumsmedizin, Greifswald, Germany

${ }^{13}$ Science-Consulting in Diabetes $\mathrm{GmbH}$, Science-Consulting in Diabetes $\mathrm{GmbH}$, Neuss, Germany

Einleitung

Diabetes mellitus ist eine Volkskrankheit, die daher - neben der individuellen Bedeutung für die betroffenen Patienten - einen großen gesellschaftspolitischen Stellenwert besitzt. Trotz intensiver Bemühungen der behandelnden Ärzte und erheblicher Fortschritte in Diagnostik und Therapie ist ein relevanter Anteil der Patienten nicht optimal therapiert, so dass immer noch ein erheblicher Handlungsbedarf besteht. Mit Gründung der Kommission für Labordiagnostik in der Diabetologie (KLD) gibt es nun ein Gremium innerhalb der Deutschen Diabetes Gesellschaft (DDG) und der Deutschen Gesellschaft für Klinische Chemie und Laboratoriumsmedizin (DGKL) für methodische Aspekte und deren klinische Konsequenzen bei laboratoriumsmedizinischen Messgrößen für die Diagnostik Behandlung von Patienten mit Diabetes mellitus.

Methoden

Diabetologie und Laboratoriumsmedizin sind eng miteinander verflochten. Nur mit einer hohen Qualität laboratoriumsmedizinischer Messergebnisse können klinisch tätige Ärzte eine zuverlässige Diagnose stellen und adäquate Therapieentscheidungen treffen. Da Messgüte und Messgenauigkeit erheblich von der Leistungsfähigkeit der eingesetzten Geräte sowie der richtigen Handhabung der Messsysteme abhängt, ergeben sich zahlreiche Aufgaben für die KLD:

- Verbesserung der Messgüte bei der Analyse von Glukose, HbA1c und weiteren relevanten Laborparametern in der Diabetologie durch

- Aktualisierung der Praxisempfehlungen zur Diabetesdiagnostik

- Kongressteilnahmen und Kommunikation der Themen

- Gremienarbeit 
- Dialog mit Herstellern von Messsystemen

- Erstellung von Stellungnahmen zu aktuellen Themen

- Evaluierung und Positionierung von klinisch-chemischen "Surrogat"-Parametern

- Organisation von Fortbildungen zum Thema Labordiagnostik, sowohl für Ärzte als auch für deren Teams

- Ansprechpartner für Fragestellungen in diesem Themenbereich, bei praktischen, klinischen und wissenschaftlichen Aspekten

- Beschäftigung mit kritischen (gesundheits-) politischen Aspekten

In dieser Kommission sind Vertreter der DGKL und der DDG paritätisch vertreten. Sie stammen aus Klinik und Labor, sowohl aus dem ambulanten als auch dem stationären Bereich und zeigen alle ein ausgeprägtes Interesse an der Wissenschaft.

\section{Ergebnisse}

Die KLD hat seit ihrer Gründung neun Publikationen erarbeitet und durch die unterstützenden Arbeiten erheblich zur Verschärfung der Qualitätskriterien beim HbA1c in der überarbeiteten Rili-BÄK beigetragen (Reduktion der Grenzen für die interne Qualitätssicherung von $\pm 10 \%$ auf $\pm 3 \%$ ). Damit konnten aufgrund der medizinischen Anforderungen die notwendigen Qualitätskriterien für die interne und externe Qualitätskontrolle definiert werden.

Diskussion und Ausblick

Die Kommissionsarbeit ist ein gelungenes Beispiel für einen sachlichen und konstruktiven Dialog zwischen unterschiedlichen Fachgebieten der Medizin. Damit trägt die KLD dazu bei, die medizinische Qualität der Krankenversorgung unmittelbar zu erhöhen und nachhaltig zu verbessern. 


\title{
PO-70
}

\section{Reference intervals for total serum calcium in seniors: results from the prospective SENIORLAB study}

\author{
Andreas Hemmerle'; Araceli Jarquin Campos²; Ursula Amstutz'; Urs Nydegger ${ }^{3}$; Benjamin Sakem; \\ Lorenz Risch ; Martin Risch ${ }^{5}$ \\ ${ }^{1}$ Center of Laboratory Medicine, University Hospital, Inselspital, and University of Bern, University \\ Institute of Clinical Chemistry, Bern, Switzerland \\ ${ }^{2}$ Private University in the Principality of Liechtenstein, Laboratory Medicine, Vaduz, Liechtenstein \\ 3 labormedizinisches zentrum Dr. Risch, Core Labor, Liebefeld, Switzerland \\ ${ }^{4}$ Labormedizinisches zentrum Dr. Risch, Core Lab, Liebefeld, Switzerland $3 / a b o r m e d i z i n i s c h e s$ \\ zentrum Dr. Risch, Klinische Chemie, Liebefeld, Switzerland \\ ${ }^{5}$ Kantonsspital Graubünden, Central Laboratory, Chur, Switzerland
}

Background and aim: Calcium represents the most ubiquitous mineral in the human body. Several important functions are attributed to calcium, such as in bone metabolism, muscle contraction, nerve conduction, and clotting of blood. As a symptom, disordered calcium homeostasis is relatively frequently encountered in elderly persons and can indicate underlying disease or drug therapy. The aim of the present analysis was to determine RI for calcium in Caucasian individuals aged 60 years and more.

Methods: From a total of 1467 study participants reporting subjective wellbeing at baseline examination in the SENIORLAB study, serum samples were drawn in fasting state and processed under optimal and standardized preanalytical circumstances. The following individuals were excluded from analysis: death at first follow-up for participants $<80$ years of age (mean follow-up time $3.7+/$ 0.7 years), survival of less than 3 years between age $80-84$, survival of less than 2 years between age $85-89$, and survival of less than 1 year for age 90 and older. Further exclusion criteria known to affect calcium levels were: systemic inflammation (CRP $>10 \mathrm{mg} / \mathrm{L}$ ), thyroid disease (TSH less than 0.35 or more than $4.94 \mathrm{U} / \mathrm{L}$ ), decreased kidney function (eGFR $<60 \mathrm{ml} / \mathrm{min} / 1.73 \mathrm{~m} 2$ ), non-adequate vitamin $D$ levels $(<20$ or $>100 \mathrm{ng} / \mathrm{mL}$ ) dysproteinemia (total protein $<60$ or $>80 \mathrm{~g} / \mathrm{L}$ ), parathyroid dysfunction (PTH $<1.6$ or $>7.2 \mathrm{pmol} / \mathrm{L})$. Further, patients with calcium supplementation, calcium antagonist medication, neoplastic disease, and a history of osteoporosis were also excluded. Effects of age and gender on calcium levels were investigated. Double sided $95 \% \mathrm{RI}$ together with the $90 \%$ confidence intervals $(\mathrm{Cl})$ were evaluated according to CLSI guideline EP28-3c.

Results: A total of 459 study participants (243 males, 216 females, age range 60-94, median age 69 years) had measurements available and were included into the present analysis. No age dependence on calcium levels could be observed. Median calcium concentrations were significantly lower in male than in female study participants ( $2.38 \mathrm{vs} 2.40 \mathrm{mmol} / \mathrm{L} ; \mathrm{p}=0.003)$. For males $(\mathrm{n}=239)$, an age independent robust RI was $2.2190 \% \mathrm{Cl}$ [ $2.20,2.23$ ] to $2.556190 \% \mathrm{Cl}$ [ 2.54,2.57 ] mmol/L. For females ( $n=216)$, an age independent robust $\mathrm{RI}$ was $2.2490 \% \mathrm{Cl}$ [ 2.22,2.26 ] to $2.554790 \% \mathrm{Cl}$ [ $2.54,2.57$ ] mmol/L. A gender and age independent robust $\mathrm{RI}(\mathrm{n}=454)$ was $2.2290 \% \mathrm{Cl}$ [ 2.21,2.23 ] to $2.5590 \% \mathrm{Cl}[2.54,2.56$ ] $\mathrm{mmol} / \mathrm{L}$.

Conclusion: In this carefully selected reference population of seniors, calcium concentrations exhibit a gender- but not age specific behavior. The lower limit of the $\mathrm{Rl}$ is $0.03 \mathrm{mmol} / \mathrm{L}$ lower in males than in females, whereas a common upper limit of the RI can be set at $2.56 \mathrm{mmol} / \mathrm{L}$. 


\title{
PO-71
}

\section{S3-Leitlinie zu Colitis ulcerosa - klinische Erfahrungen mit der Beurteilung von Cyclosporinspiegeln}

\author{
Katharina Koch"; Anne-Katrin Maier²; Bernd Köhrer² \\ 'Städtisches Klinikum Karlsruhe, ZLMT - Abteilung für medizinische Diagnostik, Karlsruhe, Germany \\ ${ }^{2}$ Städtisches Klinikum Karlsruhe, Medizinische Klinik II - Gastroenterologie, Hepatologie und \\ Diabetologie, Karlsruhe, Germany
}

Die Colitis ulcerosa ist eine vergleichsweise häufige chronisch-entzündliche Darmerkrankung. Die Deutsche Gesellschaft für Gastroenterologie, Verdauungs- und Stoffwechselkrankheiten hat daher im Mai 2018 eine Leitlinie herausgebracht, die als S3-Leitlinie der höchsten Qualitätsstufe entspricht.[1] In dieser Leitlinie wird beschrieben, dass Calcineurin-Inhibitoren (z. B. Cyclosporin A) als RescueTherapie bei steroidrefratären Verläufen der Colitis ulcerosa eingesetzt werden. Hier wird beschrieben, dass sich eine Dosis von $2 \mathrm{mg} / \mathrm{kg}$ Körpergewicht als Standard durchgesetzt hat. Obwohl erwähnt wird, dass die optimalen therapeutischen Serumspiegel nicht bekannt sind, wird ein anzustrebender Spiegel von 250 bis $400 \mathrm{ng} / \mathrm{ml}$ (gemessen als Talspiegel) angegeben. Demgegenüber steht die Fachinformation zu Cyclosporin[2], in der ebenfalls eine Dosis von 2 mg/kg Körpergewicht nach z. B. Nierentransplantation angegeben wird. Die hier angegeben Referenzbereiche liegen aber bei 100 bis $150 \mathrm{ng} / \mathrm{ml}$ (gemessen als Talspiegel). [3, 4]

Um Klarheit in die Frage der Referenzbereiche für Cyclosporinspiegel bei Patienten mit Colitis ulcerosa zu bringen, haben wir aus dem Patientenkollektiv der gastroenterologischen Stationen unseres Klinikums die Akten all derjenigen Patienten herausgesucht, die in den letzten 5 Jahren tatsächlich wegen einer Colitis ulcerosa mit Cyclosporin behandelt wurden. Dieses Patientenkollektiv ist mit sieben Patienten zu klein, um wirklich repräsentativ zu sein, dennoch können die gemessenen Cyclosporinspiegel mit den Angaben zur Dosierung des Medikaments verglichen werden. Als Methode zur Bestimmung der Cyclosporinspiegel wurde das LC-MS/MS-Verfahren verwendet, das von der Firma Chromsystems entwickelt und validiert wurde.

Eine Dosierung von etwa $2 \mathrm{mg} / \mathrm{kg}$ Körpergewicht Cyclosporin scheint demnach mit dem aus der Organtransplantation bekannten Spiegeln zu korrelieren. Ein Zielspiegel von 100 bis $150 \mathrm{ng} / \mathrm{ml}$ scheint daher sinnvoll zu sein. Auffällig war, dass es offensichtlich schwierig ist, Patienten mit Colitis ulcerosa auf einen stabilien Cyclosporinspiegel einzustellen. Es lassen sich aber Rückschlüsse von der Stabilität des eingestellten Cyclosporinspiegels auf das Ansprechen der Patienten auf die Therapie schließen. Zusammenfassend lässt sich sagen, dass auch im Falle der Gabe bei Colitis ulcerosa eine sorgfältige Überwachung des Cyclosporinspiegels unerlässlich ist, wobei die S3-Leitlinie der Deutsche Gesellschaft für Gastroenterologie, Verdauungs- und Stoffwechselkrankheiten hinsichtlich der Angaben zu den Zielkonzentrationen überarbeitet werden sollte.

Literatur:

[1] DGVS, „S3-Leitlinie - Colitis ulcerosa“, AWMF-Registriernummer: 021-009, Mai 2018.

[2] Fachinformation Ciclosporin, Rote Liste Service GmbH, Frankfurt.

[3] Gressner AM, Arndt, T, „Lexikon der Medizinischen Laboratoriumsdiagnostik“, 2. Aufl., Springer Medizin Verlag Heidelberg, 2013.

[4] Thomas, L (Hrsg.), „Labor und Diagnose“, 8. Aufl., Verlag TH-Books, Frankfurt/Main, 2012. 


\title{
PO-72 / FV-05
}

\section{Mass spectrometry based quantification of plasmaproteins}

\author{
Rupert Schreiner'; Peter Findeisen²; Nina Scheffe ${ }^{2}$ : Maria-Christina Muth ${ }^{2}$ \\ ${ }^{1} M V Z$ Labor Dr. Limbach, HPLC, Heidelberg, Germany \\ ${ }^{2} M V Z$ Labor Limbach Heidelberg, Entwicklung, Heidelberg, Germany
}

Introduction: Immunoassays are prone to unspecific interferences that might lead to false results. In contrast, mass spectrometry can overcome these shortcomings and is heralded as reference method. To test this hypothesis, we have selected five high- to medium-abundant plasmaproteins that were quantified by mass spectrometry an d commercially available immunoassays in parallel. Serum specimens form healthy controls, diabetic- and uremic patients were analyzed to evaluate the impact of post-translational proteinmodifications (glucosylation, carbamylation) on respective results.

Materials and Methods: A liquid chromatography / mass spectrometry (LC-MS/MS) based workflow was developed for the quantification of albumin, alpha-1-antitrypsin, haptoglobin, complement factor C3 and transferrin. Quantotypic peptides were selected from a publicly available database (www.srmatlas.org) and stable isotop labeled peptides were used as internal standards. Several validation parameters including dynamic range, LOD (lower limit of detection) and LOQ (lower limit of quantification), selectivity and reproducibility were evaluated. Anonymized left-over serum specimens form three collectives (healthy controls, diabetic patients and patients with chronic kidney disease) were analyzed and results were compared to commercially available immunoassays.

Results: Test characteristics (LOD, LOQ, linearity and CV) of the MS-based methods were within the expected ranges and comparable to those of respective immunoassays. Furthermore, there were no significant differences, when MS- and IA-results of healthy controls were compared. However, MSand IA-results from diabetic and uremic patients were significantly different.

Conclusion: The development of MS-based reference methods for plasmaproteins in a routine laboratory is feasible due to a comprehensive, publicly available database (www.srmatlas.org) that summarizes key features for setup of respective assays. Post-translational proteinmodifications have a strong impact on immunoassays whereas MS-based assays are unaffected. It can be anticipated that MS-based reference methods will greatly improve the standardization of protein-quantification methods 
PO-73

\title{
Apolipoprotein E phenotyping via tandem mass spectrometry
}

\author{
Benedikt Zöhrer1; Ronny Baber²; Uta Ceglarek'; Julia Dittrich¹
}

'Universitätsklinikum Leipzig, Institut für Laboratoriumsmedizin, Klinische Chemie und Molekulare Diagnostik, Leipzig, Germany 2Leipzig Research Center for Civilization Diseases, Leipzig Medical Biobank, Leipzig, Germany

Purpose: Polymorphic apolipoprotein $\mathrm{E}$ (apoE) that is located on chylomicrons, very-low density lipoproteins, and intermediate density lipoproteins plays an important role in lipoprotein metabolism. The three isoforms apoE3 (Cys112, Arg158), apoE2 (Cys112, Cys158), and apoE4 (Arg112, Arg158) are associated with differential risk for hypercholesterolemia and Alzheimer's Disease. So far, APOE genotyping is used to identify respective alleles $(\varepsilon 2, \varepsilon 3, \varepsilon 4)$. By contrast, we assessed the potential of combined quantitative apoprotein profiling and apoE phenotyping by coupling of liquid chromatography and tandem mass spectrometry (LC-MS/MS).

Method: ApoE phenotyping was performed from EDTA plasma via analysis of four isoform-specific tryptic peptides. Therefore, a validated LC-MS/MS assay for apolipoprotein quantification was expanded. For method validation, results of apoE phenotyping and APOE genotyping, performed by real-time fluorescence PCR from isolated DNA, were compared for 504 subjects of the Leipzig LIFEHeart Study.

Result: The APOE genotyping and apoE phenotyping results matched for $96.8 \%$ of the investigated subjects. For 16 discrepant cases, a second PCR analysis was performed. Respective results of both PCR analyses differed for 10/16 samples. For apoE phenotyping, the frequency distribution of the three homozygous and three heterozygous phenotypes was comparable to published results. As expected, E2/E2 was the phenotype with the highest median total apoE concentration $3.5 \mu \mathrm{mol} / \mathrm{L}$, whereas E4/E4 showed the lowest median concentration of $0.9 \mu \mathrm{mol} / \mathrm{L}$.

Conclusion: We successfully integrated apoE phenotyping to a multiplexed MS-based quantitative apoliprotein assay. In future, this technique might be a valuable tool for early risk assessment of cardiovascular and neurodegenerative diseases. 


\title{
PO-74
}

\section{The effect of blood sampling and preanalytical processing on human $\mathrm{N}$-glycome}

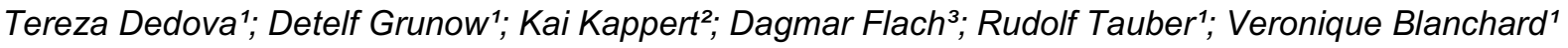 \\ ${ }^{1}$ Charité - Universitätsmedizin Berlin, corporate member of Freie Universität Berlin, Humboldt- \\ Universität zu Berlin, and Berlin Institute of Health, Institute of Laboratory Medicine, Clinical Chemistry \\ and Pathobiochemistry, Institute of Laboratory Medicine, Clinical Chemistry and Pathobiochemistry, \\ Berlin, Germany \\ ${ }^{2}$ Charité - Universitätsmedizin Berlin, corporate member of Freie Universität Berlin, Humboldt \\ Universität zu Berlin, and Berlin Institute of Health, Institute of Laboratory Medicine, Clinical Chemistry \\ and Pathobiochemistry, Institute of Laboratory Medicine, Clinical Chemistry and Pathobiochemistry, \\ Berlin, Germany \\ ${ }^{3}$ Sarstedt AG\&Co, Nümbrecht, Germany
}

Glycome modulations have been described in the onset and progression of many diseases. Thus, many studies have proposed glycans from blood glycoproteins as disease markers. Astonishingly, little effort has been given unraveling preanalytical conditions potentially influencing glycan analysis prior to blood biomarker studies. In this work, we evaluate for the first time the effect of hemolysis, storage and blood collection, but also influence of various times and temperatures between individual processing steps on the total $\mathrm{N}$-glycome and on a glycan-biomarker score. Venous blood was collected from 10 healthy donors in 11 blood collection tubes with different additives, processed variously to obtain 16 preanalytical variables and $\mathrm{N}$-glycans released from serum or plasma were analyzed by MALDI-TOFMS and capillary electrophoresis coupled with fluorescence detection (CE-LIF) for the first time. Long time storage of deep frozen samples at $-20^{\circ} \mathrm{C}$ or $-80^{\circ} \mathrm{C}$ exerted only a minor influence on the glycome as demonstrated by CE-LIF. The N-glycome was very stable evidenced by MALDI-TOF when stored at $4^{\circ} \mathrm{C}$ for at least 48 hours and blood collected in tubes devoid of additives. The glycome was stable upon storage after centrifugation and aliquoting, which is an important information considering future diagnostic applications (1). Hemolysis, however, negatively correlated with an established glycan score for ovarian cancer, when evaluated by MALDI-TOF-MS measurement by affecting relative intensities of certain glycans, which could lead to false negative / positive results in glycan biomarker studies $(2,3)$.

1 Dedova, T., et al. (2018) "The effect of blood sampling and preanalytical processing on human N-glycome". Plos One 13, e0200507.

2 Biskup, K., et al. (2013) "Serum Glycome Profiling: A Biomarker for Diagnosis of Ovarian Cancer". J Proteome Res 12, 4056-4063.

3 Biskup, K., et al. (2014). The serum glycome to discriminate between early-stage epithelial ovarian cancer and benign ovarian diseases. Disease markers 2014, 238197. 


\section{PO-75}

\section{Comparison of 1H NMR metabolic profiles between serum, EDTA-plasma and lithium-heparin- plasma}

Friederike Gauß ${ }^{1}$; Kathrin Budde ${ }^{1}$; Ann-Kristin Henning ${ }^{1}$; Nele Friedrich ${ }^{1 ;}$ Astrid Petersmann ${ }^{1}$; Maik Pietzner ${ }^{1}$; Matthias Nauck ${ }^{1}$

${ }^{1}$ Universitätsmedizin Greifswald, Institut für Klinische Chemie und Laboratoriumsmedizin, Greifswald, Germany

Objective:

A common difference between research and clinical practice is the type of material used for analysis. EDTA-plasma is primarily used in research settings, whereas in clinical routine serum is preferable. The aim of the present study was to investigate whether metabolic profiles obtained by $1 \mathrm{H}-\mathrm{NMR}$ spectroscopy are comparable between these blood specimens as step towards translation into the clinic.

Methods:

Blood samples were collected from 100 patients at the hospital of the University Medicine Greifswald. All patients provided serum, EDTA- and lithium-heparin plasma samples. In all samples metabolic profiles were measured by $1 \mathrm{H}-\mathrm{NMR}$ spectroscopy using a 600Mhz device. Comprehensive, quantitative lipoprotein subfraction $(n=112)$ and plasma metabolite $(n=19)$ data were obtained.

Equality of measurements for each of the variables across the specimens was compared using Passing-Pablok regression and Pearson correlation coefficients.

Results:

All lipoproteins and most plasma metabolites were highly correlated between the three specimens. The median correlation coefficient was 0.99 (IQR: $0.99-1.0$ ) and 0.95 (IQR: $0.87-0.98$ ), respectively. The comparisons serum/EDTA-plasma and lithium-heparin/EDTA-plasma showed, however, a tendency towards lower correlation coefficients than the comparison between serum and lithium-heparin-plasma. Lowest inter-fluid correspondence $(r<0.8)$ was observed for citrate across all specimens as well as histidine and pyruvic acid for the comparisons serum/EDTA-plasma and lithiumheparin/ EDTA-plasma, respectively.

Passing-Bablok regression revealed no or only small proportional bias for most of the analytes: the median slope was 0.97 (IQR: 0.95 - 0.99) for lipoproteins and 1.0 (IQR: 0.97 - 1.0) for plasma metabolites, respectively. About one third of the lipoproteins showed a bias between all three specimens, e.g. Apolipoproteins $\mathrm{A} 1$ and $\mathrm{A} 2$, but none of the plasma metabolites. Most analytes with a proportional bias were found in the serum/EDTA-plasma comparison.

\section{Conclusion:}

We examined the differences of $1 \mathrm{H}-\mathrm{NMR}$ metabolic profiles in the three blood specimens serum, lithium-heparin plasma and EDTA-plasma. Our results showed high correlation coefficients between all specimens, with a better correlation between serum and lithium-heparin plasma as opposed to EDTA-plasma. Most plasma metabolites and lipoproteins showed no or only small proportional bias in the comparison for small molecules. 


\title{
PO-76
}

\section{Determination of the 2-hydroxyglutarate oncometabolite enantiomers}

\author{
Gry Helene Dihazi'; Frank Streit'; Tatjana Khromov²; Catharina Cohrs²; Lutz Binder ${ }^{2}$ \\ ${ }^{1}$ UMG-Göttingen, Institut für Klinische Chemie/ UMG-Labor, Göttingen, Germany \\ ${ }^{2}$ Universitätsmedizin Göttingen, Institut für Klinische Chemiel UMG-Labor, Göttingen, Germany
}

2-Hydroxyglutarate (2-HG), with its two enantiomers D- and L-2-HG, is a potent competitor of $\alpha$ ketoglutarate $(\alpha-K G)$ and may impact histone and DNA demethylation. It was first associated with 2hydroxyglutaric aciduria, an inherited 2-hydroxyglutarate dehydrogenase (2- $\mathrm{HGDH})$ deficiency, which is associated with brain damage in children. 2-HGDH converts $2-\mathrm{HG}$ into $\alpha-K G$ and its deficiency leads to an accumulation of $2-\mathrm{HG}$, particularly the $\mathrm{L}$ enantiomer, which may also contribute to an elevated cancer risk. More recently, it was shown that L-2-HG may accumulate in various cell types, particularly under hypoxic or acidic mitochondrial conditions. Furthermore, isocitrate dehydrogenase (IDH1/2) mutants, which are found in different malignancies such as glioma, acute myeloid leukemia, head and neck carcinoma and colorectal cancer, produce D-2-HG from $\alpha-K G$. This leads to an increasing interest in this oncometabolite.

For this purpose, differential detection of L-2-HG and D-2-HG is needed - for body fluids, cells and tissues (Struys et al, 2004; Oldham and Loscalzo, 2016). Previously described methods show insufficient sensitivity in plasma and only limited throughput. The aim of this project was to establish a sensitive high throughput method for differentiation and quantification of the 2-HG enantiomers in different materials.

Blood donors donated EDTA plasma and serum samples for method validation. To quantitate the 2HG enantiomers, a protocol from Oldham and Loscalzo (2016) was adapted using diacetyl-L-tartaric anhydride (DATAN). In order to increase the sensitivity of the method and sample preparation throughput, certain changes were made. Briefly, $100 \mu \mathrm{l}$ of supernatant of the precipitated sample was added to $5 \mu \mathrm{l}$ of $10 \mu \mathrm{M}$ internal standard in a 96-well plate. The organic solvent was removed in a positive pressure manifold (PPM) prior to derivatization with $5 \mu$ l of DATAN. After incubation at $70{ }^{\circ} \mathrm{C}$, the solvent was removed by the PPM. The dried samples were reconstituted with $100 \mu \mathrm{l}$ of $0.1 \%$ formic acid before being quantified with a Xevo TQ-S (Waters) equipped with a chromatographic column. The mobile phases were $0.2 \%$ formic acid $(A)$ and methanol $(B)$. The data were acquired in negative mode and analyzed with MassLynx software (Waters).

Derivatization of the enantiomers with DATAN made it possible to separate chromatographically and to quantify the derivatives. Inter- and intraassay CVs were within the criteria of FDA and EMA and the detection limit of the given method was $2 \mathrm{nmol} / /$. The concentration of the L-2-HG and D-2-HG enantiomers, as well as their ratio, was stable for more than $4 \mathrm{~h}$ at RT. The improved sample preparation enabled a high throughput workflow of plasma and serum samples as well as cell lysates.

We established and optimized a sensitive, fast and reliable high throughput method for the differentiation and quantification of the 2-HG enantiomers for the use in scientific, clinical and diagnostic questions. 


\title{
Onkologie, Hämatologie und Hämostaseologie -1
}

\author{
PO-77 / FV-02
}

\section{Diagnostic approach to von Willebrand disease with special consideration of von Willebrand factor propeptide (vWF:pp): a new, rapid and specific vWF:pp ELISA}

\author{
Oliver Tiebel'; Thomas Wellhöfer²; Hans- Jürgen Kolde; Beate Krammer-Steiner'; Jan Lüdemann; \\ Michael Steiner ${ }^{5}$
}

\author{
${ }^{1}$ Universitätsklinikum Carl Gustav Carus Dresden, TU Dresden, Institut für Klinische Chemie und \\ Laboratoriumsmedizin, Dresden, Germany \\ ${ }^{2} \mathrm{fzmb} \mathrm{GmbH}$, Forschungszentrum für Medizintechnik und Biotechnologie, Bad Langensalza, \\ Germany \\ ${ }^{3}$ Consulting Diagnostics, Seefeld, Germany \\ ${ }^{4}$ Klinikum Südstadt Rostock, Klinik für Innere Medizin III, Rostock, Germany \\ ${ }^{5}$ Medizinisches Labor Rostock, Rostock, Germany
}

Background \& Aim: In 2015 Sanders et al. reported, that von Willebrand factor propeptide (vWF:pp) identified severe type 1 von Willebrand disease (vWD) with very low von Willebrand factor (vWF) levels in patients who had previously been classified as type 3 vWD underlining the diagnostic significance of vWF:pp in classifying vWD patients. 1 In 2018 O'Sullivan noted that enhanced vWF clearance may play an important role in the pathogenesis of VWD and has therapeutic relevance. 2 Currently available colorimetric ELISA-based vWF:pp assays are rather complicated and can not be completed within $24 \mathrm{~h}$. In contrast, a rapid, specific and simple ELISA that could be easily implemented into routine laboratory diagnostic procedures was recently developed. 3

Methods: A sandwich type ELISA was designed based on precoated ready-to-use strips with a total assay time of about $90 \mathrm{~min}$. The new assay exhibits a detection limit of $1.7 \mathrm{mlU} / \mathrm{ml}$ and a broad measurement range $(1.7-120 \mathrm{mIU} / \mathrm{ml})$. The intra-assay precision $(\mathrm{CV})$ was $8.1 \%$ at $3.8 \mathrm{mIU} / \mathrm{ml}$ and $0.1 \%$ at $60.6 \mathrm{mIU} / \mathrm{ml}$. The inter-assay precision (8 days, CV) was $9.8 \%$ at $3.8 \mathrm{mIU} / \mathrm{ml}$ and $0.6 \%$ at $60.6 \mathrm{mIU} / \mathrm{ml}$. Evaluation based on samples from healthy controls and vWD patients revealed a good correlation with the Sanquin assay $(y=0.91 x+4.43) .3$ During a clinical evaluation period, the assay was applied on blood specimen of 17 vWD patients (low von Willebrand factor $(n=5)$, type $1(n=7)$, type $1 \mathrm{C}$ $(n=3)$, type $3(n=2))$.

Results: vWF:pp was below the limit of detection in the cases of vWD type 3. As expected, all vWD type 1C patients were characterised by a markedly increased vWF:pp/vWF:Ag-ratio. Within the vWD type 1 group, three cases demonstrated a vWF:pp/vWF:Ag-ratio $<2.0$ and 4 cases a ratio $>2.0$. In all LWF-cases the vWF:pp/vWF:Ag-ratio was $<2.0$.

Conclusions: The newly developed ELISA with a short assay and less hands-on time, ready-to-use microtiter strips, and good performance characteristics enables an integration of vWF:pp into established vWD diagnostic work-up algorithms. The vWF:pp/vWF:Ag-ratio appears to be helpful in discriminating patients with a markedly increased vWF clearance (acquired vWD or vWD type 1 Vicenza) from those with a modest increased clearance (most vWD patients).4,5 Information about clearance defects may have direct therapeutic implications.6 DDAVP is the treatment of choice in VWD type 1 patients, but increasing the endogenous VWF levels by regular DDAVP dosing may not be sufficient in patients with a major VWF clearance defect.6 Identification of VWD patients with enhanced vWF clearance may contribute to optimized therapeutic regimens.

\section{References:}

1) Sanders YV et al. Blood. 2015;125:3006

2) O'Sullivan JM et al. Br J Haematol. 2018;183:185

3) Wellhöfer T et al. Hamostaseologie. 2019:39(S01):S1-S92

4) Haberichter SL et al. Blood. 2008;111:4979 
5) Stufano F et al. Semin Thromb Hemost. 2019;45:36

6) De Jong A \& Eikenboom J J Thromb Haemost. 2016;14:449 


\title{
PO-78 / PV-13
}

\section{Platelet-derived growth factor receptor $\beta$ regulation during development of myelofibrosis}

\author{
Frederike Kramer'; Jens Dernedde'; Artur Mezheyeuski²; Rudolf Tauber; Patrick Micke²; Kai Kappert ${ }^{3}$
}

${ }^{1}$ Charité-Universitätsmedizin Berlin, Institut für Laboratoriumsmedizin, Klinische Chemie und Pathobiochemie, Berlin, Germany

${ }^{2}$ Uppsala University, Department of Immunology, Genetics and Pathology, Uppsala, Sweden ${ }^{3}$ Labor Berlin - Charité Vivantes GmbH, Laboratoriumsmedizin und Toxikologie, Berlin, Germany

Background: Several lines of evidence suggest platelet-derived growth factors (PDGFs) and their receptors in the development of primary myelofibrosis (PMF). Indeed, PDGF receptor $\beta$ (PDGFR $\beta$ ) expression has been demonstrated being increased in bone marrow stromal cells of patients with PMF and correlating with the grade of myelofibrosis. However, the specific role of PDGFR $\beta$ signaling in PMF has not been determined.

Aim: Thus, here we analyzed in-depth the dynamics of PDGFR $\beta$ signaling using the Gata-1low mouse model for PMF.

Methods: We applied a combination of RNAsequencing, qPCR, protein expression analyses, multispectral imaging and, as a novel approach in bone marrow tissue, an in situ proximity ligation assay (PLA) to characterize PDGFR $\beta$ signaling and regulation during the development of myelofibrosis.

Results: We detected an increase in both PDGFR $\beta$ and PDGF-B gene and protein expression in fibrotic bone marrow of mice at 15 months of age. This was accompanied by an increase in PDGFR $\beta-$ PDGF-B interaction as determined by PLA. However, tyrosine phosphorylation of the PDGFR $\beta$, analyzed by PLA, was not elevated. We thus analyzed the regulation of PDGFR $\beta$ by protein tyrosine phosphatases (PTPs) as endogenous antagonists of the PDGFR $\beta$. PDGFR $\beta$-targeting PTPs showed distinct gene expression dynamics. Especially T-cell PTP (TC-PTP) gene and protein expression, as well as PDGFR $\beta$-TC-PTP interaction was enhanced in early bone marrow fibrosis in mice at the age of 10 months as well as in overt fibrotic bone marrow of Gata-1low mice at 15 months. TC-PTP (Ptpn2) knockdown in fibroblasts in vitro increased phosphorylation of PDGFR $\beta$ on Y751 and Y1021, subsequently leading to elevated phosphorylation of AKT and PLCY1. In addition, Ptpn2 knockdown lead to increased cellular growth rates under low-serum conditions.

Conclusions: In conclusion, PDGFR $\beta$ signaling is highly and differentially regulated during development of experimental PMF. Further, PTPs, not being analyzed during disease progression previously, are suggested as novel signaling components affecting PMF. 


\title{
PO-79 / PV-14
}

\section{Methylation in the Phospholipase A2 Receptor Promoter Region in Childhood Acute Lymphoblastic Leukaemia}

\author{
${ }^{1}$ Markus Friedemann; Katharina Gutewort'; Dana Thiem²; Carsten Jandeck ${ }^{3 ;}$ Björn Sönke Lange ${ }^{2}$; \\ Meinolf Suttorp ${ }^{2}$; Mario Menschikowski ${ }^{1}$ \\ 'Uniklinik Dresden, Institut für Klinische Chemie und Laboratoriumsmedizin, Dresden, Germany \\ ${ }^{2}$ Uniklinik Dresden, Department of Pediatrics, Dresden, Germany \\ ${ }^{3}$ Uniklinik Dresden, Institut für Klinische Chemie und Laboratoriumsmedizin, Dresden, Germany
}

Background: Acute lymphoblastic leukaemia (ALL) is the most common form of paediatric cancer and despite improving cure rates, ALL remains the leading cause of death from cancer during childhood. Epigenetic changes are considered as an important determinant for leukemogenesis.

Aims: The aim of the present study was to investigate the methylation degree of a distinct phospholipase A2 receptor 1 (PLA2R1) promoter region in patients with childhood ALL evaluating this parameter as possible new biomarker for the control of treatment response and residual disease. The impact of PLA2R1 expression on cellular proliferative behaviour was assessed in vitro after transfection of childhood Jurkat ALL cells in which the PLA2R1 gene is naturally silenced due to DNA methylation.

Methods: Genomic DNA from bone marrow aspirates (BM) and peripheral blood samples (PB) of 44 childhood ALL patients was isolated and modified by bisulfite treatment. PLA2R1 promotor methylation was analysed by droplet digital PCR and compared to healthy individuals. Human childhood acute leukaemia (Jurkat) cells were transfected to overexpress PLA2R1 and the effect on cell proliferation and apoptosis/ necrosis was investigated by cell growth curve analysis and flow cytometry in vitro.

Results: The PLA2R1 promoter was found to be hypermethylated in BM and PB samples of childhood patients with primary pre-B and common ALL at diagnosis as well as in patients with relapse. Levels of PLA2R1 methylation decreased in parallel to leukemic blast cell reduction during ALL induction treatment. Re-analysis of Illumina Infinium 450k Human DNA methylation data confirmed PLA2R1 promoter hypermethylation as an important epigenetic aberration across different subtypes of childhood ALL. In vitro analysis revealed a pro-apoptotic/necrotic and anti-proliferative phenotype associated with PLA2R1 re-expression in Jurkat cells.

Conclusions: According to the observed data, the determination of PLA2R1 promoter methylation appears to be a suitable biomarker for the control of induction treatment response and early detection of relapses in childhood acute leukemic patients. The in vitro results showed that a silencing of PLA2R1 by DNA methylation facilitates a cellular apoptosis/necrosis escape connected with an upregulated proliferation of leukemic blast cells that illustrates the receptor as tumour suppressor. 
PO-80

\title{
Comparison of three serum free light chain assays - heterogeneity and implications on diagnosis and treatment of multiple myeloma
}

\author{
Sebastian Hörber'; Aneta Schieferdecker²; Monika Ums³; Britta Besemer; Carsten Bokemeyer²; Katja \\ Weisel2; Andreas Peter $^{1}$ \\ ${ }^{1}$ University Hospital Tübingen, Institute for Clinical Chemistry and Pathobiochemistry, Tübingen, \\ Germany \\ University Medical Center Hamburg (UKE), Department of Oncology and Hematology, Hamburg, \\ Germany \\ ${ }^{3}$ University Hospital Tübingen, Department of Hematology, Oncology, Immunology, Rheumatology and \\ Pulmonology, Tübingen, Germany
}

Background: Quantification of serum free light chains (FLC) is an essential part of the diagnosis and management of patients with multiple myeloma (MM). According to current guidelines, an involved FLC/non-involved FLC (iFLC/niFLC) ratio of $>100$ is defined as threshold for patients with MM requiring treatment. This threshold is based on values determined by the Freelite assay (The Binding Site). In addition, quantification of FLC can be performed using the N Latex FLC assay (Siemens Healthineers) or the recently launched Sebia FLC assay (SEBIA).

Study design and methods: Blood samples were collected from 47 patients with newly diagnosed $(n=31)$ or relapsed $(n=16)$ MM at the beginning of the study and in a maximum of six follow-up visits. In total, serum free light chains $(\mathrm{K}, \lambda$ and $\mathrm{K} / \lambda$ ratio) were determined in 187 serum samples. Quantification was performed using Freelite and N Latex FLC reagents on a BNII nephelometer (Siemens Healthineers). Sebia FLC, based on solid phase sandwich enzyme-linked immunosorbent technology (ELISA), was performed manually. Results were compared between assays and evaluated according to diagnostic thresholds.

Results: Overall, correlation between results of all three FLC assays was good, at least for quantification of $K F L C$ and calculation of $\kappa / \lambda$ ratio. $\lambda F L C$ values showed high variability between all assays. KFLC concentrations determined by N Latex FLC and Sebia FLC were lower in contrast to $\lambda F L C$ concentrations showing higher values compared to Freelite results. Consequently, $k / \lambda$ ratios of the Freelite assay were higher in comparison to both other assays. Evaluation of assay-specific reference ranges revealed good concordance for $K F L C$ and $k / \lambda$ ratio, but low concordance for $\lambda F L C$ for all three assays. Regarding the $\mathrm{iFLC/niFLC}$ ratio $>100$, all assays showed poor concordances.

Conclusion: Here we report on the first study comparing results of the currently available FLC assays in patients with MM. We show heterogeneity of single FLC values demonstrating that FLC assays should not be used interchangeably. Especially, diagnosis and monitoring of patients with MM are affected by the choice of FLC assay. Furthermore, iFLC/niFLC ratios differed considerably between all assays. Therefore, the iFLC/niFLC ratios for N Latex FLC and Sebia FLC should be adapted. 


\title{
PO-81
}

\section{Establishment of Age and Gender Specific Reference Intervals of 29 Routine and 13 Research Use Only CBC parameters on Abbott Alinity hq Hematology Analyzer}

\author{
Ludi van Dun'; Kristin Thorausch²; Peter Thorausch²; Gernot Musikowski; Claudia Naparty²; Zainab \\ Mukhtar ${ }^{1}$
}

\section{${ }^{1}$ Abbott Diagnostics, Hematology, Wiesbaden-Delkenheim, Germany} ${ }^{2}$ MVZ Gemeinschaftslabor Cottbus, Hematology, Cottbus, Germany

Introduction:

\begin{abstract}
Alinity hq is the recently launched fully automated Abbott Hematology System which works on the principle of Advanced MAPSS Technology incorporating both light scatter (optical) and fluorescence flow cytometry principles. It has 7 optical and 1 fluorescence detector which reports $C B C+6$ Part Differential (with IGs) and Reticulocyte parameters, without interference from Resistant RBC's and also reports Nucleated RBCs with every $C B C+$ Diff result. The aim of this study was to establish reference intervals for all CBC+Diff+Retic parameters (both Reportable and RUO). Secondary aims were to investigate gender dependency of the reference ranges.
\end{abstract}

\section{Method:}

During the first 6 weeks of 2018, blood samples that were routinely processed in the MVZ Gemeinschaftslabor Cottbus laboratory were run in the CBC+Diff mode including reticulocytes. Blood was drawn into BD and Sarstedt evacuated tubes containing K2-EDTA as an anticoagulant and the tubes were kept at ambient temperature for no longer than 6 hours after collection. A population of 2177 unique subjects (1116 male and 1061 female) was used for this study, reference ranges were calculated. The central 95th percentile ranges were calculated according to the CLSI guideline with parametric method along with non-parametric method and a applying the Bhattacharya statistics, with linear regression fit line as it was general practitioner patient population and assumption was that this population is less homogeneous than the healthy donor population. In order to exclude selection bias, reference ranges were also calculated for a subset of 1724 individuals (781 male and 943 female) who were expected to be not iron-deplete.

Results:

Reference values have been defined and statistically significant gender difference was observed in $\mathrm{RBC}, \mathrm{HGB}, \mathrm{HCT}$ and $\mathrm{cHGB}$.

\section{Conclusion:}

The reference values of blood cell count established by the present study constitute an important tool of results interpretation in our laboratory. However, these reference intervals we recommend should be validated by individual laboratories for the local population as recommended by CLSI. 


\title{
PO-82
}

\section{Von Willebrand disease diagnostics: only the selection of an appropriate diagnostic armament ensures a conclusive differentiation of vWD}

\author{
Oliver Tiebel'; Karolin Trautmann-Grill2; Ralf Knöfler ${ }^{3}$; Sophie Bierkämper; Gabriele Siegert ${ }^{4}$ \\ 'Universitätsklinikum Carl Gustav Carus Dresden, TU Dresden, Institut für Klinische Chemie und \\ Laboratoriumsmedizin, Dresden, Germany \\ ${ }^{2}$ Universitätsklinikum Carl Gustav Carus an der Technischen Universität Dresden, Medizinische Klinik \\ 1, Dresden, Germany \\ ${ }^{3}$ Universitätsklinikum Carl Gustav Carus an der Technischen Universität Dresden, Klinik und Poliklinik \\ für Kinder- und Jungendmedizin, Dresden, Germany \\ ${ }^{4}$ Universitätsklinikum Carl Gustav Carus an der Technischen Universität Dresden, Institut für Klinische \\ Chemie und Laboratoriumsmedizin, Dresden, Germany
}

Background \& Aim: Von Willebrand disease (vWD) is the most common inherited bleeding disorder. Appropriate diagnosis of VWD, including differentiation of qualitative vs. quantitative defects has important implications, but remains challenging. 1 Diagnostic differentiation will become increasingly important as more personalized approaches to manage patients with vWD become available, particularly recombinant von Willebrand factor concentrate.2 The minimal requirement for proper identification of VWD includes assessment of F.VIII, vWF Antigen (VWF:Ag) and both vWF collagen binding function (VWF:CB) and VWF platelet adhesion binding (VWF:RCo) - or the increasingly used direct ristocetin-induced recombinant GPIbR binding (vWF:GPIbR). 3

The classic functional VWF:RCo assay is prone to high imprecision and low sensitivity. The new generation tests assessing platelet function of VWF offer more reliable results. According to the current nomenclature they are able to differentiate between VWF:GPIbR and VWF:GPIbM (spontaneous binding of vWF to a gain-of-function mutant GPIb fragment). 4

Methods: 308 samples from vWD-patients including type 1,2 and 3 vWD were systematically evaluated for vWF:Ag, vWF:CB and vWF:GPIbR applying HemosIL AcuStar-Assays (Instrumentation Laboratory, Werfen Group, Kirchheim). For all samples vWF:CB/Ag- as well as vWF:GPIbR/Ag-ratios were calculated.

Results: In total we identified 23 vWD type 2M constellations in 13 patients. 7 results (4 patients) disclosed a suspect vWF:CB/Ag-ratio - without loss of large vWF multimers. 16 results (9 patients) revealed abnormal vWF:GPIbR/Ag-ratios. For 6 out of these 9 patients regular vWF multimer patterns were ascertained so far. Without applying the VWF:GPIbR assay we would have misinterpreted 6 patients -1 patient would have been classified as type $1 \mathrm{VWD} ; 5$ patients as low vWF. More importantly, in 3 patients the diagnosis of VWD would have been missed completely, since they all showed normal results for VWF:Ag, vWF:CB and vWF:CB/Ag-ratios.

Conclusion: According to Doruelo et al. clinical laboratory variability may reflect imperfect assays, such that some variants may be alternately classified as either type $2 \mathrm{~A}$ or type $2 \mathrm{M} .5$ From our experience and the current set of data con- respectively discordant VWF:CB/Ag- and VWF:GPIbR/Ag-ratios guide to a coherent classification of type $2 \mathrm{~A} / 2 \mathrm{~B}$ and $2 \mathrm{M}$ cases. So vWF multimer distribution may not be required necessarily in all cases for finalising a diagnosis.

Applying an incomplete initial set of diagnostic tests creates a substantial risk to miss VWD cases to a certain extend. Our data strongly support the recommendation of Favaloro regarding the minimum requirements for an adequate identification of VWD.3

References:

1) Favaloro EJ et al. Haemophilia. 2016;22:e145

2) Favaloro EJ et al. Semin Thromb Hemost. 2016;42:483 
3) Favaloro EJ Am J Hematol. 2017;92:114

4) Bodo I et al. J Thromb Haemost. 2015;13:1345

5) Doruelo AL et al. J Thomb Haemost. 


\title{
PO-83
}

\section{Vollautomatisierte Quantifizierung von inhibitorischen Antikörpern gegen Gerinnungsfaktoren}

\author{
Thomas Siegemund ${ }^{1 ;}$ Annelie Siegemund ${ }^{1 ;}$ Hagen Bönigk ${ }^{1}$
}

${ }^{1}$ MVZ Limbach Magdeburg, Magdeburg, Germany

Problemstellung

Erkennung und Quantifizierung von inhibitorischen Antikörpern gegen Gerinnungsfaktoren sind mit einem hohen technischen Aufwand verbunden und bedürfen hochqualifiertes Personal. Die Methode, der Bethesda-Assay, weißt eine hohe Variabilität auf, unter anderem durch eine hohe Anzahl an manuellen Prozessschritten.

Eines der wichtigsten Hindernisse in der Automatisierung ist die in der Methode geforderte zweistündige Inkubationszeit von Antikörper mit dem Antigen. In theoretischen Untersuchungen der Reaktionskinetik im Bethesda-Assay kann gezeigt werden, dass diese für Gerinnungsfaktoren mit Ausnahme von Faktor VIII deutlich verkürzt werden kann. Weiterhin wird ein neuer, deutlich vereinfachter mathematischer Ansatz zur Quantifizierung der Inhibitorstärke vorgestellt.

\section{Material und Methoden}

35 Proben von Patienten, die routinemäßig auf Inhibitoren untersucht werden wurden in die Studie eingeschlossen. Darüber hinaus, wurden kommerzielle erworbene Inhibitorplasmen untersucht. Die Automatisierung des Bethesda-Assays erfolgte am Atellica COAG360 Analzyer (Siemens Healthineers). Für Antikörper gegen folgende Gerinnungsfaktoren wurde die Methode getestet: Faktor VII (Bethesda Assay), Faktor V (Bethesda, besierend auf PT und aPTT) und Faktor IX (Bethesda- und Nijmegen Methode). Verdünnungsstufen zwischen 1:2 und 1:256 wurden realisiert. Restaktivität des Gerinnungsfaktors und korrespondierende Inhibitorstärke werden vom Analyzer selbst errechnet.

Eine einzelne Bestimmung benötigt etwa 17 Minuten, in einem Fall wurden 14 gleichzeitig platzierte Proben in je vier Verdünnungstufen innerhalb von ca. 90 Minuten abgearbeitet. Der Reagenzverbrauch im Bethesda-Assay ist identisch mit dem manuellen Assay, im Nijmegen-Assay, insbesondere für Mangelplasma, sogar deutlich reduziert.

\section{Ergebnisse}

Die im automatisierten Assay erzielten Inhibitorstärken sind vergelichbar denen im manuellen Assay. Alle inhibitorpositiven Proben wurden als positiv bestimmt, die beobachteten Abweichungen der Inhibitorstärke lagen unterhalb von 30\%. Mit einer Ausnahme wurden alle negativen Proben als negativ bestimmt. Der Ausreißer wurde durch das Vorliegen eines starken Lupus-Antikoagulanz bedingt.

\section{Diskussion}

Vollautomatisierte Bestimmung von inhibitorischen Antikörpern gegen Gerinnungsfaktoren führt zu einer deutlichen Verminderung des technischen Aufwandes und bedarf keiner zusätzlichen Qualifizierung von Personal. Weiterhin eröffnet sie einen Weg zur weiteren Standardisierung der Methode und ermöglicht eine Reduktion der Inter-Labor-Variabilität. Positive Resultate bedürfen einer zusätzlichen Validierung durch die Bestimmung des Lupus-Antikoagulanz.

Eine Adaptation der Methodik auf andere vollautomatische Gerinnungssysteme (BCS, ACLTOP oder STA-Serie) ist in Vorbereitung. 


\title{
PO-84
}

\section{Optimization of sample preparation for analysis of intracellular signaling proteins in platelets}

\author{
Katharina von Bargen 1; Franziska Knüttgen²; Tobias Flieder'; Monika Wolny'; Anne-Kathrin Vollmer2; \\ Wiebke Kreuzberg'; Stefan Wiese ${ }^{3}$; Cornelius Knabbe ${ }^{2}$; Ingvild Birschmann ${ }^{2}$
}

${ }^{1}$ Herz- und Diabeteszentrum Nordrhein-Westfalen, Institut für Laboratoriums- und

Transfusionsmedizin, Bad Oeynhausen, Germany

${ }^{2}$ Institut für Laboratoriums- und Transfusionsmedizin, Herz- und Diabeteszentrum Nordrhein-

Westfalen, Universitätsklink der Ruhr-Universität Bochum, Bad Oeynhausen, Germany, Institut für

Laboratoriums- und Transfusionsmedizin, Bad Oeynhausen, Germany

${ }^{3}$ Fakultät für Biologie und Biotechnologie, Ruhr-Universität Bochum, Arbeitsgruppe Molekulare

Zellbiologie, Bochum, Germany

Objective:

Within platelets, there are numerous signaling pathways that have not yet been completely understood in detail. To further characterize such pathways, signaling proteins within the cell can be analyzed.

Since platelets do not have their own expression system, analysis of signaling proteins is often based on their post-translational modifications. An example of this is the tyrosine kinase Src. This signaling protein is regulated by phosphorylation or dephosphorylation on conserved tyrosine residues. Comparison of inactivated and activated platelets allows further analysis of Src-induced signaling pathways.

In this study we wished to establish an optimal sample preparation to show a comparison of intracellular signaling proteins before and after activation of platelets.

\section{Methodology:}

In the study we activated washed platelets with various agonists (i.e. TRAP, ADP and fibrinogen) during sample preparation. Following that, the cells were lysed by different reagents to achieve an optimal detection of intracellular proteins by Western blot analysis. To examine the cell lysis, the amount of protein was then determined by BCA assay. Subsequently, the proteins were enriched by TCA precipitation and used for Western blot analysis (detection of cytosolic GAPDH, membrane protein GPIIla, Src).

\section{Findings:}

The first results of our study showed a significant protein loss after platelet activation (detected by BCA-Assay). The main problems were adhesion of activated platelets to the walls of the laboratory vessels and insufficient lysis of activated and aggregated platelets. Consequently, various conditions were tested. For example, the platelets were activated at different times during sample preparation. In addition, different combinations of activators and lysis buffers were tested. In several experimental approaches, a significant improvement could be detected. The previously observed protein loss could be significantly reduced. In addition, to assess the different cell lysis, various proteins were detected by Western blot (cytosolic GAPDH, membrane protein GPIIla, Src). These results also showed a significant improvement in sample preparation.

\section{Conclusion:}

To analyze intracellular signaling proteins of platelets we optimized sample preparation to avoid protein loss and to achieve sufficient cell lysis, even of activated cells. To this end, various activation conditions and cell lysis were tested. It was possible to significantly optimize the sample preparation. Now we are able to compare intracellular signaling proteins, such as $\mathrm{Src}$, of resting and activated platelets. 


\section{Onkologie, Hämatologie und Hämostaseologie -2}

\section{PO-85}

\section{Comparison of HM3 and HVAD patients with respect to different clinical chemical and platelet function parameters: a pilot study}

Tobias Flieder'; Franziska Knüttgen'; Katharina von Bargen"; Monika Wolny'; Anne-Kathrin Vollmer'; Thomas Eller'; Cornelius Knabbe ${ }^{1}$; Ingvild Birschmann ${ }^{1}$

${ }^{1} H e r z-$ und Diabeteszentrum Nordrhein-Westfalen, Universitätsklinik der Ruhr-Universität Bochum, Institut für Laboratoriums- und Transfusionsmedizin, Bad Oeynhausen, Germany ${ }^{2}$ Labor Krone, Bad Salzufflen, Bad Salzufflen, Germany

Aim

Implantation of a left ventricular assist device (LVAD) is the last therapy option for patients with terminal heart failure, besides HTx. Due to the lack of donor organs, however, the number of patients receiving $L V A D$ as a bridge to transplantation is increasing. In our clinic we examined patients with HeartWare (HVAD) and HeartMate 3 (HM3) systems, which differ in their pump functions, and compared them regarding various parameters.

\section{Methodology}

In our clinic, 15 patients with an HVAD and 8 patients with an HM3 system were included in the study. Clinical chemistry, hematologic and hemostaticologic parameters were measured and, in particular, the platelet function was considered. These were determined by aggregation according to Born and impedance aggregation.

\section{Results}

The results of the clinical chemical parameters were striking for their significantly lower values for cholinesterase and albumin in patients with HM3 compared to the HVAD patients. As expected, platelet function was pathologically aggregated in patients under aspirin (ASA) therapy, except for one patient who showed aspirin resistance. However, even in patients without ASA therapy, platelet function was partially reduced. In the range of hemostaseological parameters, the values for FVIII and VWF Ag and VWF activity were significantly higher for patients with HM3

\section{Conclusions}

The examination of patients with HM3 and HVAD showed differences in some laboratory parameters. The HM3 system, which is supposed to generate fewer shear forces than the HVAD system, nevertheless shows a clear limitation in platelet function even without aspirin therapy. The extent to which the differences in cholinesterase, albumin, FVIII and VWF parameters can be attributed to the implanted system must be investigated with larger collectives. 


\section{PO-86}

\section{Complement C3a-chain shows different sizes in plasma, platelets and other sources}

Franziska Knüttgen'; Katharina von Bargen²; Tobias Flieder2; Monika Wolny2; Anne-Kathrin Vollmer2; Cornelius Knabbe ${ }^{2}$; Ingvild Birschmann ${ }^{2}$

${ }^{1}$ Herz- und Diabeteszentrum Nordrhein-Westfalen, Universitätsklinik der Ruhr-Universität Bochum, Institut für Laboratoriums- und Transfusionsmedizin, Bad Oeynhausen, Germany

${ }^{2}$ Institut für Laboratoriums- und Transfusionsmedizin, Herz- und Diabeteszentrum Nordrhein-

Westfalen, Universitätsklink der Ruhr-Universität Bochum, Bad Oeynhausen, Germany, Institut für Laboratoriums- und Transfusionsmedizin, Bad Oeynhausen, Germany

Objective: Overlaps between the complement system and coagulation form a research focus that is receiving increasing attention. Since there is a higher risk of hemorrhages at sites of infection, as well as a higher risk of infection at locations of bleeding, these overlaps seem reasonable and unsurprising. In this context, the platelets play a central role. It has already been demonstrated that platelets are able to initiate the classical pathway of complement activation and to directly cleave the complement component C3. Furthermore, platelets can lead to phosphorylation of C3b, and, in turn, $\mathrm{C} 3 \mathrm{~b}$ can bind to P-selectin on the platelet surface. In addition, it has been shown by mass spectrometry and Western blot that platelets themselves contain complement components, such as C3. The following is a closer look at C3 from platelets.

Methods: For the examination of platelet complement C3a, well washed platelets were prepared. Triton and Tween were used to lyse the cells. To enrich or purify C3, immunoprecipitation was performed with anti-C3 antibody coated Dynabeads. The coated beads were then incubated with lysates or plasma, washed, and the bound proteins eluted with SDS sample buffer. Platelet, HEK 293 , human liver whole tissue lysate and plasma samples were applied to an $8 \%$ SDS gel for Western blot analysis. For the detection of $\mathrm{C} 3 \alpha$ via a specific anti-C3 antibody, the separated proteins were blotted onto nitrocellulose. Sequence analyses of cDNA from different sources were performed.

Results: In order to avoid the contamination of platelets C3 with C3 from plasma, platelet samples must be carefully cleaned of plasma residues. Albumin was considered to be a measure for the sufficient purity of the samples, being no longer detectable in the supernatant of the platelet samples after five washes. In order to detect C3a in platelets, it was necessary to enrich C3a by immunoprecipitation since platelets contain only small amounts of this protein. The antibody used was able to detect C3a in platelets, HEK293 cells, liver lysate and plasma. The detected C3a from different sources showed different sizes. Liver and plasma C3a show the same size and are the largest overall. $\mathrm{C} 3 \alpha$ from platelets is slightly smaller, while C3a from HEK 293 cells has the smallest size. Sequencing of the C3 cDNA from different sources revealed some SNPs, which mainly lead to silent mutations. In HepG2 two, and in platelets three heterozygous SNPs were found that lead to missense mutations.

Conclusion: Depending on its source, $\mathrm{C} 3 \alpha$ shows different sizes in Western blot analysis. The reason is unknown so far. In order to find the underlying cause, complete sequencing of $\mathrm{C} 3$ from various cDNA sources was performed. There were no sequence differences that could explain the different sizes in the C3a chain. Mass spectrometric tests will be carried out for further investigations of this topic and will be discussed on the poster. 


\title{
PO-87
}

\section{Prevalence of EDTA-Anticoagulation Associated Platelet Agglutination in a Large Out-Patient Laboratory: Specificity and Sensitivity of Sysmex XE 5000 Flagging for Platelet Aggregates}

\author{
Joe Mansour'; Steffen Mannuß; Angela Gropp1; Klaus Scharfenberg'; Peter Schuff-Werner ${ }^{3}$ \\ ${ }^{1} \mathrm{MDI}$ Labor Limbach Berlin - Medical Care Center -, Hämatologie, Berlin, Germany \\ ${ }^{2}$ University of Applied Sciences Emden-Leer, Dept. of Natural Science Technology, Emden, Germany \\ ${ }^{3}$ University Medical Center Rstock, Hematology - Clinical Chemistry, Rostock, Germany \\ Introduction: \\ Whole blood anti-coagulated with ethylene-diamine-tetra-acetate (EDTA) is a necessary pre-requisite \\ for blood cell counting, but this leads in 0,01 to $0,2 \%$ of the patients samples to a time- and \\ temperature-dependent in vitro aggregation of platelets, resulting in falsely low platelet counts and \\ therefor medically wrong conclusions. This phenomenon, named pseudo-thrombocytopenia (PTCP) \\ requires the detection of spontaneous platelet aggregation already during automatic blood count \\ analysis.
}

\section{Study design:}

Using our routine hematology analyzer (Sysmex XE 5000) CBCs from 1000 EDTA-anticoagulated blood samples from unselected patients were measured in the order of their arrival in the laboratory. In parallel blood smears were produced and microscopically analyzed for platelet aggregate formation.

CBC flags, abnormalities of either the IMI channel and / or the platelet distribution curve indicating platelet aggregate formation were compared with the result of the microscopic analysis of the blood smears. The results were compared for calculating sensitivity, specificity and efficiency of different indicators for platelet aggregates.

Results:

Pseudo-thrombocytopenia was found in 6 out of 977 patients, a prevalence of $0.61 \%$. Only two of the 6 PTCP were flagged by the respective aggregate warning. Other warnings of the device that are reported to be associated with platelet aggregates were also found unsuitable to safely identify PTCP, as shown by the calculation of diagnostic specificity, sensitivity and efficiency.

The prevalence of EDTA-induced platelet agglutination in a cohort of unselected out-patients was

$0.6 \%$ and therefore in accordance with the literature. All 6 cases were identified by microscopic examination of the blood films (number of really positive cases). Only two cases were flagged by the hematology analyzer XE 5000 (sensitivity 33\%; specificity 97\%). The analysis of the IMI channel resulted in the identification of 5 out of the 6 positive samples (sensitivity 83\%, specificity $63 \%$ ). Characteristic changes of the declining portion of the platelet distribution curve ("saw tooth similar") were also found in 5 out of the 6 positive samples (sensitivity $83 \%$, specificity $43 \%$ ).

\section{Conclusions:}

Our study revealed a $0.61 \%$ prevalence of pseudo-thrombocytopenia in out-patients of a large centralized laboratory. The XE 500 analyzer flags as well as the separate analysis of IMI channel and /or the platelet distribution curve are not very helpful for searching out platelet aggregates in EDTAanticoagulated blood. 


\section{PO-88}

\section{Analytical criteria determine SCD40L detection}

Folker Wenzel'; Saskia Mayer'; Frithjof Blessing²

${ }^{1}$ Hochschule Furtwangen, Medical and Life Sciences, Villingen Schwenningen, Germany 2Laborärzte Singen, Labor, Singen, Germany

Introduction: CD40 Ligand is a multifunctional ligand of the tumour necrosis superfamily with activated platelets as major source. The SCD40L basal level could be used as a biomarker. Because of difficulties with preanalytical detection of SCD40L levels it is necessary to standardize the determination of SCD40L under clinical as well as experimental conditions.

Material and Methods: Serum and plasma samples of healthy blood donors $(n=15)$ were used to determine $\mathrm{SCD} 40 \mathrm{~L}$ concentrations under different conditions, SCD40L basal levels and the maximum release of SCD40L. Those conditions were different anticoagulants, different storing temperatures and different timepoints of centrifugation. For detection of SCD40L levels an Enzyme-linked immunosorbent Assay Kit for SCD40L was used.

Results: The determined mean value of the basal $\mathrm{sCD} 40 \mathrm{~L}$ (obtained at $\mathrm{t}=0 \mathrm{~min}$ ) was $451.2 \mathrm{pg} / \mathrm{mL}$. The basal levels reached from $114.3 \pm 56.7 \mathrm{pg} / \mathrm{mL}$ (inhibitor treated citrate samples) to $620.1 \pm 161.6$ $\mathrm{pg} / \mathrm{mL}$ (L-Heparin samples). The serum samples stored at room temperature or $37^{\circ} \mathrm{C}$ showed a steady increase of SCD40L concentration over time. While the concentration of the samples stored at $37^{\circ} \mathrm{C}$ showed a maximum range of 10000 to $14000 \mathrm{pg} / \mathrm{mL}$ after $120 \mathrm{~min}$, there is no maximum seen by the samples stored at room temperature.

Conclusion: SCD40L levels could be used as a clinical parameter but it depends on analytical conditions. Thus, a standardization of storing conditions and detection in general is necessary to prevent the blood samples from blood clotting and activation of platelets which are the major source of SCD40L in peripheral blood. Following studies should examine the interaction between platelets more precisely, SCD40L and matrix-metalloproteinases (MMP) to generate diagnostical options for SCD40L. 


\title{
PO-89
}

\section{Validation of the PAGE-B score for HCC risk assessment in patients with chronic hepatitis B receiving antiviral therapy}

\author{
Martin Sprinzl'; Arndt Weinmann²; Peter R. Galle²; Karl J. Lackner \\ 'Universitätsmedizin Mainz, Department of Clinical Chemistry and Laboratory Medicine, Mainz, \\ Germany \\ ${ }^{2}$ Universitätsmedizin Mainz, Department of Medicine I, Mainz, Germany
}

Background: Early diagnosis of hepatocellular carcinoma (HCC) is crucial, as only early stages of HCC may allow curative therapy. Sonographic HCC surveillance has therefore been established in chronic hepatitis $\mathrm{B}(\mathrm{cHB})$ infected patients at risk to develop HCC. Additional risk stratification by the PAGE-B score, however, has identified cHB patients with a low cumulative HCC risk, who might not require HCC surveillance (1). We therefore introduced the PAGE-B score algorithm in our laboratory information system and evaluated the PAGE-B score in a retrospective manner.

Patients and Methods: Patients with cHB attending the Medical University Center, Mainz, were retrospectively analyzed between 1992 and 2018. Demographic and laboratory data were retrieved from the hospital information system (i.s.h.med®, SAP) and the laboratory information system (Swisslap $®$, Lauris ${ }^{\circledR}$ ), respectively. The PAGE-B score was applied in cHB-patient receiving treatment with nucleos(t)ide analogues (NA) (entecavir or tenofovir) for a minimum of one year (1). Patients were subsequently categorized into a low ( $\leq 9$ pts.), intermediate (10-17 pts.) and high ( $\geq 18$ pts.) HCC risk group, according to PAGE-B. Variables are provided in rates (\%) or median (interquartile range, IQR) if not specified otherwise.

Results: A total of $n=1336 \mathrm{cHB}$ patients were identified, who attended our medical center between 1992 and 2018. Among these cHB patients $n=322$ patients were eligible for PAGE-B-based HCC risk assessment. This subgroup was characterized by the following variables: Age 51.5 years (42.0-61.5 years), female gender (68\%), anti-HBc-Ab positive (100\%), HBeAg positive (18.9\%), HBV-DNA negative (74.5\%), serum ALT $30 \mathrm{U} / \mathrm{L}(20-42 \mathrm{U} / \mathrm{L})$, serum albumin $40 \mathrm{~g} / \mathrm{L}(38-42 \mathrm{~g} / \mathrm{L})$, total bilirubin 0.62 $\mathrm{mg} / \mathrm{dl}(09.44-1.01 \mathrm{mg} / \mathrm{dl})$ and thrombocytes $212 \times 10 \mathrm{e} / \mathrm{L}$ (166-263 x10e9/L). HCCs were diagnosed in $\mathrm{n}=28(8.7 \%)$ patients after initiation of NA therapy. PAGE-B stratification identified patients with low $(n=89,27.6 \%)$, intermediate $(n=165,51.2 \%)$ and high $(n=68,21.1 \%)$ HCC risk. The PAGE-B risk groups correlated with cumulative HCC rates of $0.0 \%, 10.3 \%$ and $16.2 \%$, respectively (log rank, $\mathrm{p}=0.004)$.

Conclusion: The PAGE-B score can identify a subgroup of cHB patients with no relevant cumulative 5year HCC probability in our cohort. The PAGE-B score was subsequently implemented via an algorithm of the laboratory information system for routine use, which helps to tailor the sonographic HCC screening to the individual needs of each cHB patient under antiviral treatment.

Reference: (1) Papatheodoridis G. et al. PAGE-B predicts the risk of developing hepatocellular carcinoma in Caucasians with chronic hepatitis B on 5-year antiviral therapy. Journal of hepatology 2016; 64:800-806. 
PO-90

A panoramic view on RNA-binding proteins controlling the hemostatic system

Ranya Al Shekaki'; Jamie Nourse'; Ansgar Poetsch²; Sven Danckwardt ${ }^{1}$

${ }^{1}$ Universitätsmedizin Mainz, CTH Mainz Institut für klinische Chemie und Laboratoriumsmedizin, Mainz, Germany

${ }^{2}$ University of Plymouth, School of Biomedical Sciences, Plymouth, United Kingdom

RNA-binding proteins (RBPs) are central for gene expression by controlling the RNA fate from birth to decay. Various disorders arising from perturbations of RNA-protein interactions document their critical function.

Despite the involvement of RBPs in virtually all biological processes, and the importance of the hemostatic system for a multitude of actions in and beyond blood coagulation, the role of postgenomic gene regulation in hemostasis is poorly defined. Here we set out to comprehensively illuminate RBPmediated regulation of the hemostatic system in an unbiased manner.

We used an unbiased screening approach that employs RBP pull-downs coupled to mass spectrometry involving 20 hemostasis-associated gene 3' untranslated regions (UTRs). Examination of this panel revealed 757 UTR/RBP interactions involving 151 RBPs. Among various interesting candidates, we identify PABPC4, KHSRP and CIRBP as surprisingly selective RBPs controlling the expression of specific hemostatic components. This includes components of the intrinsic system (FXI) currently emerging as novel therapeutic targets for safe anticoagulants.

This study highlights the regulatory role of RBPs in hemostatic control. It contributes to the interdisciplinary endeavor to identify novel therapeutic targets in the fight against thromboembolism with minimal risk of bleeding. 


\title{
PO-91
}

\section{Vergleich zweier Methoden zur Bestimmung der freien Leichtketten im Serum}

\author{
Carola Charlotte Feier ${ }^{1}$; Kornelia Hummel2; Klaus-Martin Otte ${ }^{2}$; Hans Salwender ${ }^{3}$ Sandra Job²
}

${ }^{1}$ Asklepios Medical School GmbH, Campus Hamburg, Hamburg, Germany

${ }^{2}$ Medilys Laborgesellschaft, Klinische Chemie, Hamburg, Germany

${ }^{3}$ Asklepios Klinik Altona, Abteilung für Onkologie mit Sektion Hämatologie, Hamburg, Germany

\section{Einführung}

Die Bestimmung der freien Leichtketten Kappa und Lambda im Serum sowie dem Quotienten Kappa/Lambda sind essentielle Marker zur Diagnose und Verlaufsbeurteilung beim Multiplen Myelom, insbesondere beim Leichtkettenmyelom. Auf der Grundlage der Höhe der Messwerte und des Quotienten Kappa/Lambda werden therapeutische Entscheidungen getroffen. Standardverfahren zur Bestimmung der freien Leichtketten sind die Turbidimetrie (THE BINDING SITE GmbH, Schwetzingen) sowie die Nephelometrie. Als weiteres Testverfahren ist ein ELISA (Fa. Sebia GmbH, Fulda) kürzlich auf den Markt gekommen. In dieser Untersuchung wurde der ELISA Test der Fa. Sebia auf dem AP22 Elite (Fa Sebia) mit dem turbidimetrischen Test der Fa. THE BINDING SITE auf dem SPAPLUS (Fa. THE BINDING SITE) miteinander verglichen. Das Ziel der Untersuchung war die Korrelation und die Höhe der Wertelage dieser beiden Teste zu ermitteln.

\section{Material und Methoden}

Für den Vergleich wurde die Lambda-Leichtkette von 108 und die Kappe-Leichtkette von 100 Patienten gemessen. Der Wertebereich betrug für den FreeliteR Immunoassay bei der Lambda-LK 0,47 mg/l bis $17221 \mathrm{mg} / \mathrm{l}$, bei der Kappa-LK 0,41 mg/l bis $16921 \mathrm{mg} / \mathrm{l}$

Ermittelt wurde die Korrelation und die Wertelage, dargestellt im Bland Altman-Plot.

Im FreeliteR Immunoassay wurden bei einigen Patienten sehr hohe Messwerte gefunden, die aber kein entsprechendes Korrelat in der Elektrophorese oder Immunfixation hatten.

\section{Ergebnisse}

Die statistische Auswertung erfolgte mit dem Programm Abacus 2.0. Die Korrelation für die KappaLeichtkette betrug nach Pearson 0,876 (0,821-0,915), für die Lambda-Leichtkette (LK) 0,902 (0,859 0,932 ). Die Geradensteigung nach Passing-Bablok betrug für die Kappa-LK 0,273, für die Lambda-LK 0,361 .

Im Bland Altman Plot zeigte sich sowohl für die Kappa-LK, als auch für Lambda-LK ein kontinuierliches Auseinanderdriften der Wert, abhängig von der Konzentration. Je höher die Werte beim FreeliteR Immunoassay lagen, umso mehr differierten sie zum ELISA-Test.

\section{Zusammenfassung}

Der Vergleich beider Testverfahren zur Bestimmung der freien Leichtketten zeigt bei höheren und hohen Wertelagen stark differierende Ergebnisse mit deutlich höheren Werten in der Turbidimetrie. Als Ursache kann man Polymerisationen der freien Leichtketten und der damit gestörten turbidimetrischen Messung vermuten. Die Messungen mit der ELISA- Technik scheint für dieses Phänomen unempfindlicher zu sein.

Die Messwerte der freien Leichtketten stellen eine wichtige Grundlage für therapeutische Entscheidungen z.B. zur High Cut-Off Dialyse (HCO-Dialyse) dar. Die unterschiedlichen quantitativen Messwerte im Vergleich zeigen, dass die Messergebnisse kritisch zu hinterfragen sind und ggf. weitere Methoden wie Elektrophorese und Immunfixation sowie die Plausibilität zum klinischen Verlauf gleichwertig in die Entscheidungsfindung einbezogen werden müssen. 


\title{
Eröffnungsveranstaltung
}

\section{V-01} Festvortrag: Der Mensch im Bauhaus. Menschliche Proportionen und Wahrnehmung als
Gestaltprinzip für Architektur und Stadtplanung

\author{
Johannes Ringe/ ${ }^{1}$ \\ 1 Universität Leipzig Direktor des Instituts für Stadtentwicklung und Bauwirtschaft an der \\ Wirtschaftswissenschaftlichen Fakultät der Universität Leipzig, Leipzig, Germany
}

„Der Mensch im Bauhaus. Menschliche Proportionen und Wahrnehmung als Gestaltprinzip für Architektur und Stadtplanung" | Vortrag Prof. Johannes Ringel, Universität Leipzig

Was haben Labormedizin und Bauhaus-Architektur miteinander gemein? Auf den ersten Blick nicht viel. Doch könnte dieser Erstbefund falscher kaum sein, steht doch bei beiden der Mensch im Mittelpunkt jeglichen Bemühens. So war es der berühmte Bauhaus-Schüler Le Corbusier, der mit seinem „Modulor" ein Proportionsschema entwickelt hatte, das nicht nur der Architektur eine konsequent am Menschen orientierte, mathematische Ordnung gab, sondern das auch bis heute als eine der bedeutendsten Architekturtheorien überhaupt gilt. Auch Ernst Neufert, ebenfalls BauhausSchüler der ersten Stunde, entwickelte mit seiner Bauentwurfslehre einen Standard, der menschliche Proportionen und Ergonomie in den Mittelpunkt des Bauens stellte.

Johannes Ringel, Professor für Städtebau an der Universität Leipzig, greift in seinem Impulsvortrag die Ideen und Konzepte von Le Corbusier und Neufert auf. So erläutert er, wie das Bauhaus und seine Vordenker eine neue, anthropozentrische Architektur entwickelten, die heute, im 100. Jubiläumsjahr des Bauhauses, nichts von ihrer Faszination eingebüßt hat. Sie ist die Basis für die Einbauküche im IKEA-Katalog ebenso wie für die Großbausiedlungen der Nachkriegszeit - aber auch für einen neuen Städtebau. Und sie kann uns dabei helfen, den Stadtraum der Zukunft wieder möglichst menschengerecht zu gestalten. 


\section{Proteomics}

\section{V-02}

\section{Drug Monitoring von therapeutischen Antikörpern}

\section{Nina Scheffe ${ }^{1}$}

${ }^{1}$ MVZ Labor Limbach Heidelberg, Entwicklung, Heidelberg, Germany

Therapeutische Antikörper oder andere Biologicals werden immer stärker als Medikamente eingesetzt. In Analogie zu Medikamenten mit einem kleinen Molekulargewicht ist auch hier oft während der Therapie eine Ermittlung der Wirkstoffkonzentration interessant, um Patienten zielgerichtet behandeln zu können. Allerdings unterscheidet sich das analytische Verfahren zur Spiegelbestimmung deutlich von anderen Methoden im TDM.

Aufgrund der Molekülgröße werden überwiegend Antikörper basierte Tests eingesetzt, die allerdings deutliche Probleme mit der Bestimmung haben, wenn das Immunsystem des Patienten ADAs (Anti drug related antibodies) bildet und somit die Immunoassays stört.

Die von uns verwendete Analytik nutzt die chromatographische Auftrennung und den massenspektrometrischen Nachweis der therapeutischen Antikörper zur Spiegelbestimmung. Zur Erreichung der gewünschten Spezifität werden die Proteine vor der Analyse tryptisch verdaut und über eine Festphasenextraktion aufgereinigt.

Diese Vorgehensweise ist für alle untersuchten Antikörper einheitlich und daher gut automatisierbar. Die größten Probleme ergeben sich in der unterschiedlichen Größe und Hydrophilie der Peptide, aber auch den Matrixeffekten, die in den unterschiedlichen Proben anfallen. Derzeit ist eine weitere Problematik der LCMSMS-Verfahrens, dass die Gesamtkonzentration an therapeutischen Antikörpern detektiert wird und eine Differenzierung der freien und ADA-gebundenen Antikörper noch nicht möglich ist. 


\section{V-03}

\section{Quantitative apolipoprotein profiling by LC-MS/MS-results from the Leipzig Heart study}

Julia Dittrich ${ }^{1}$

${ }^{1}$ Institut für Laboratoriumsmedizin, Klinische Chemie und Molekulare Diagnostik, Leipzig, Germany

Background and aims: Dyslipidemia is one of the major risk factors for atherosclerotic cardiovascular disease (ASCVD). Apolipoproteins (apos), which are key regulators of lipoprotein metabolism, are discussed as vascular risk factors. To elucidate the diagnostic potential of major plasma apos in cardiovascular risk assessment, we analyzed their associations with coronary artery disease (CAD), peripheral artery disease (PAD), and carotid artery plaque (CAP).

Methods: In a case-control subgroup of the Leipzig LIFE-Heart Study $(\mathrm{N}=911)$ the simultaneous quantitation of the apos A-I, A-II, A-IV, B-100, C-I, C-III, E, and J was accomplished from $3 \mu \mathrm{I}$ EDTAplasma applying LC-MS/MS. Confounder analysis with demographic and clinical covariates as well as serum lipids, cardiac, inflammatory, and hepatic markers was performed. Associations of apos with CAD, CAP, and PAD were analyzed in a multivariate regression model.

Results: Fasting and statin therapy had strongest effects on apo concentrations. Besides concentrations of apos B-100, C-I, and E also apoA-II plasma concentration was decreased under statin therapy. Further, troponin T and interleukin 6 showed inverse correlations with HDL-related apos A-I, A-II, A IV, and C-I. Solely apoB-100 (odds ratio per one SD [OR], 1.39; 95\% confidence interval $[\mathrm{Cl}], 1.05-1.84)$ was independently associated with $\mathrm{CAD}$ after adjustment for the identified influencing factors and related lipids. By contrast, only apoA IV (OR, 0.74; 95\% $\mathrm{Cl} 0.58-0.95)$ indicated PAD.

ApoB 100 (OR, 1.55; 95\% Cl, 1.18-2.04), apoC-III (OR, 1.30; 95\% Cl, 1.06-1.58), and apoE (OR, 1.34; $95 \% \mathrm{Cl}, 1.13-1.58)$ were associated with CAP.

Conclusions: Apos A-IV, B-100, C-III, and E that are associated with triglyceride-rich lipoproteins (TRL) are independently correlated with stable ASCVD, providing further evidence for a potential role of TRLs in atherogenesis. 


\title{
The future of NGS in experimental and clinical Microbiology
}

\section{V-04}

\author{
Next generation sequencing: first diagnostic one-stop shop in personalised clinical \\ microbiology
}

John Rosen ${ }^{1}$

${ }^{1}$ University of Groningen, Medical Microbiology, Groningen, Netherlands

Current molecular diagnostics of human pathogens provide limited information that is often not sufficient for outbreak and transmission investigation. In addition, when used for detection of pathogens often a biased approach is used. In this presentation an overview of the use of next generation sequencing in clinical microbiology and infection prevention will be given. Next generation sequencing (NGS) determines the DNA sequence of a complete microbial, viral or fungal genome in a single sequence run, and from these data, information on resistance and virulence, as well as information for typing is obtained, useful for outbreak investigation. The obtained genome data can be further used for the development of an outbreak-specific screening test. Like every new technology adopted in microbiology, the integration of NGS into clinical and routine workflows must be carefully managed. As the microbiology laboratories have to adhere to various national and international regulations and criteria for their accreditation, quality control issues for using WGS in microbiology, including the importance of proficiency testing, are presented. In addition, applications of NGS in the clinical setting are discussed, such as outbreak management, molecular case finding, characterization and surveillance of pathogens, rapid identification of bacteria using the 16S-23S rRNA region, taxonomy, and metagenomics approaches on clinical samples. Finally, we share our vision on the use of NGS in personalised microbiology in the near future, pointing out specific requirements. 


\section{AG Junges Labor}

\section{V-05}

\section{System diagnostics of the human gut microbiome using metaproteome analysis of fecal samples.}

Robert Heyer ${ }^{1}$

\section{${ }^{1}$ OvGU, Magdeburg, Bioprocess Engineering, Magdeburg, Germany}

A multitude of diseases are considered to be correlated with a dysbiosis of the gut microbiome, e.g. inflammatory bowel disease (IBD), diabetes, autoimmune diseases or depression. Unfortunately, up to now, it is for most diseases unclear if the dysbiosis is a cause of the disease or a result. Nevertheless, patient treatment may benefit from in-depth microbiome characterization performed in addition to the commonly associated clinical laboratory analyses enabling a more precise diagnosis of the disease or a monitoring of the disease progression. Potentially, information about the gut microbiome might even help to predict the (in)compatibility of certain drugs, enabling a more personalized patient treatment.

Technical advance of the past 10 years concerning the development of high throughput methods for the identification of genes (metagenomics/metatranscriptomics), metabolites (metabolomics) and proteins (metaproteomics) allow now a fast and reliable description of the gut microbiome. Among these methods, metaproteomics has the particular advantage that it enables both the identification of proteins from the microorganisms and from the host. Whereas the former provides information about the taxonomic composition and the functionality of the microbiome, the latter supports to draw conclusions about the patient's health.

In this presentation I will introduce a workflow for metaproteome analysis of fecal samples comprising cell lysis, protein extraction, tryptic digestion, LC-MS/MS measurement and bioinformatics data evaluation. Furthermore, I will talk about challenges and potentials of the application of fecal metaproteomics for clinical diagnosis. As examples I will present one study focusing on a weight loss program and one study including a larger cohort of patients with IBD (Ulcerative Colitis and Crohn's disease).

Due to the decrease of weight and a diet rich in fibers an improvement of the health status of the gut was observed as indicated by a decrease in the abundance of proteins assigned to the UniProtKB keywords inflammation and immune response. Furthermore, the abundance of microbial proteins related to the degradation of complex polymers increased.

In the IBD study, we could show that in feces of IBD patients IgG and neutrophil extracellular traps were significantly increased and correlated with the clinical disease activity. Furthermore, several alterations of the abundance of microbial proteins were observed such as a decrease in the abundance of the transcriptional regulatory protein RprY from Bacteroides fragilis (UniRef50_Q9AE24), which might contribute to IBD pathogenesis.

In sum, fecal metaproteomics reveals information about the health of the host and its microbiome as well as improves our understanding of the complex relationships between the microbiome and pathogenesis of several diseases. 


\title{
Biobanking: Perspektiven und Herausforderungen Gemeinsame Sitzung mit der Deutschen Gesellschaft für Pathologie (DGP)
}

\section{V-06}

\section{Biobanken-assoziierte Bedingungen und ihr Einfluss auf die Biostabilität von klinisch relevanten Biomarkern nach 5 Jahren Lagerung}

\author{
Ronny Baber \\ ${ }^{1}$ Universitätsklinikum Leipzig, Institut für Laboratoriumsmedizin, Klinische Chemie und Molekulare \\ Diagnostik, Germany
}

Biobanken können im Zeitalter der Präzisionsmedizin ein wichtiger Schlüssel für die Biomarkerforschung sein. Eine der Hauptaufgaben von Biobanken ist die Lagerung von Biomaterialien die den biologischen bzw. biochemischen Zustand des Spenders zum Zeitpunkt der Blutentnahme widerspiegeln sollen. Problematisch ist allerdings, dass selbst heutzutage wenig über den Einfluss von Biobank-assoziierten Bedingungen oder der Langzeitlagerung auf Bioproben bekannt ist bzw. diese nur unzureichend untersucht sind. Darum ist eine der wichtigen Fragen, ob Biomarker-Analysen aus in Biobanken gelagerten Proben wirklich valide sind.

Die hier vorgestellte Studie stellt die potentiellen Effekte von Biobank-assoziierten Bedingungen wie Dauer der Lagerung, Lagertemperatur, Lagerröhrchen (Kunststoffhalme, Kryoröhrchen, normale Schraubröhrchen), Füllstand der Röhrchen und Handhabungszyklen (Proben 15 min bei Raumtemperatur bzw. Temperaturen $<-100^{\circ} \mathrm{C}$ ) auf Serum und Plasma dar. Hierfür wurden EDTAPlasma-, Citrat-Plasma- und Serumproben von Patienten des Universitätsklinikums Leipzig, die nicht mehr für die Diagnostik im Institut für Laboratoriumsmedizin, Klinische Chemie und Molekulare Diagnostik benötigt wurden, gepoolt und mehr als 4.500 Aliquots für Analysen über einen Zeitraum von 10 Jahren hergestellt. Die Proben wurden in Ultratiefkühlschränken der Firma Thermo $\left(-80^{\circ} \mathrm{C}\right)$ und Askion C-line HS200 Kühltanks in der Gasphase flüssigen Stickstoffs (< - $\left.150{ }^{\circ} \mathrm{C}\right)$ gelagert. Zum Zeitpunkt des Einfrierens der Proben wurde die Basiserhebung für 58 Analyten unter Verwendung verschiedenster Analysemethoden durchgeführt. Die klinisch relevanten Biomarker sind unterschiedlichen Klassen (Elektrolyte, Enzyme, Hormone, Metaboliten, Gerinnungsfaktoren u.a.) und Organsystemen zuzuordnen.

Es werden die Ergebnisse nach 5 Jahren der Lagerung bei $-80^{\circ} \mathrm{C}$ und in der Gasphase flüssigen Stickstoffs $\left(<-150^{\circ} \mathrm{C}\right)$ dargestellt. Weiterhin wird der Einfluss der Handhabungszyklen, unterschiedlicher Lagerröhrchen und Füllstände und ihr möglicher Einfluss auf die Stabilität von Biomarkern in gelagerten Serum und Plasmaproben gezeigt. 


\section{Symposium BNLD: Wie weit geht der Verantwortungsbereich des Labors?}

\section{V-07}

\section{Patient Blood Management}

Johannes Peter Aufenanger ${ }^{1}$

${ }^{1}$ Klinikum Ingolstadt, Institut für Laboratoriumsmedizin, Ingolstadt, Germany

Since 2010, the implementation of Patient Blood Management (PBM) has been demanded by the World Health Organization.

PBM appears as an evidence-based, patient centred, multidisciplinary approach, aiming to optimise the care of patients who might need transfusion and, consequently, the use of blood products. PBM is based on three pillars: optimise the patient's own blood supplies, minimise blood loss, optimise patient's tolerance of anaemia.

The current state of data shows that unnecessarily administered blood transfusions are associated with an increase in mortality and morbidity.

At the clinical centre of Ingolstadt, we were able to reduce the consumption of packed red blood cells by approximately $50 \%$ in the period from 2010 to 2018 , mainly by consistently observing transfusion triggers. At the same time, consumption of coagulation factors (PPSB) and fibrinogen increased due to a change in coagulation management.

In orthopedic und surgical patients in particular, we were able to show the greatest success in patient blood management, not so much through the pre-treatment of iron deficiency anaemia, but through targeted indication for transfusion with the aid of transfusion triggers.

In Germany, preoperative anemia treatment has so far only been established in a few hospitals. In everyday life, the costs of intravenous iron therapy, the separation of outpatient and inpatient care and the potential postponement of the surgical intervention are often used as arguments against the preoperative treatment of anemia. Above all, the question has not been clarified as to who is medically responsible in the German health system for optimal preparation of the patient before surgery, especially in the case of high-risk surgery, and who has to bear the costs of therapy - the practioner, the referring physician, the surgeon, the anaesthesiologist or intensive care specialist or the hospital? In addition to the cost and budget aspects, preoperative anemia treatment could also be beneficial for the patient himself (less transfusion-associated risks and side effects, better healing processes), for the clinic (patient recruitment and marketing) and for the general public (shortage of blood reserves, faster rehabilitation). 


\section{Symposium BNLD: Wie weit geht der Verantwortungsbereich des Labors?}

\section{V-08}

\section{Entwicklung und Aufbau einer POCT e-Learning Lösung zur Nutzer-Rezertifizierung}

Rolf Bikker

${ }^{1}$ Medizinische Hochschule Hannover, Institut für Klinische Chemie, Hannover, Germany

Introduction

One of the main demands in POCT is efficient operator training, because insufficient training leads to many errors. Due to a variety of users, e-learning solutions are preferred. However, most commercial e-learning solutions do not consider specific work processes within the clinic which forces user frustration and may reduce training efficiency.

Methods

For creation of the e-learning modules, the ILIAS learning management system was used. Web-based learning modules and knowledge-control tests for different devices have been developed by the trainers using ILIAS author-tools. To evaluate the commercial and new e-learning modules we set up anonymous online questionnaires before and after Integration

\section{Results}

Initially the POCT-Operators were characterized a new e-learning solution was created based on operator feedback. After the implementation of new e-learning courses for Blood gas (BGA) and Blood glucose (BG) significantly better ratings in a reduced processing time in both courses were found. For the BGA course an overall improvement of evaluated categories were obtained. For the BG course a slight improvement were measured. In terms of content, the modules deal with typical sources of error. Recertification based on the test results is fully integrated in the POCT management process. After the implementation, a new evaluation survey showed significant improvements due to the new e-learning concept and a higher acceptance within the POCT-operators.

\section{Conclusion}

The development of a highly adaptable e-learning approach for the operating cycles in our hospital leads to good acceptance within the POCT operators and allows the trainer a much more flexible integrating of new upcoming POCT issues. In this study we found that it is necessary to adapt elearning courses concerning hospital issues. Commercial non adapted course courses may lead to operator frustration and unnecessary time consumption. 


\section{Glyco-based biomarkers in laboratory diagnostics}

\section{V-09}

\section{Congenital Disorders of Glycosylation (CDG)}

\section{Christian Thie/ ${ }^{1}$}

${ }^{1}$ Universitätsklinikum Heidelberg, Klinik Kinderheilkunde I, Heidelberg, Germany

Glycosylation is one of the most common modifications of proteins and lipids. The glycan moieties are important for biochemical processes such as correct folding, solubility, directed transport, activity or protection against degradation and also mediate cell-matrix and cell-cell interactions. Due to the importance of the glycans for the organism, it is not surprising that patients with deficiency in one of the glycosylation pathways usually suffer from severe clinical signs. Common symptoms comprise e.g. psychomotor and mental retardation, cerebellar hypoplasia, ataxia, seizures and muscular hypotonia as well as coagulopathy, liver and heart failure. Meanwhile the disease group 'Congenital Disorders of Glycosylation' (CDG) summarizes more than 140 different monogenetic defects within the synthesis and transfer of $\mathrm{N}-$, O- and C-glycans to glycoproteins and glycolipids. As some of the CDG defects are treatable by the simple oral supplementation of sugars, a rapid diagnostic investigation by biochemical verification of the hypoglycosylation status of serum marker proteins like transferrin or Apolipoprotein CIII via HPLC, IEF or mass spectrometry coupled with genetic analysis is essential for providing patients and their families with a reliable report. In particular, the analysis of glycans by mass spectrometry proves to be an ideal tool to monitor a patient's course of therapy. To provide an overview, we here will focus on the diagnostic procedure of CDG deficiencies and on the therapeutic outcome of selected CDG cases. 


\section{V-10}

\section{Altered glycosylation in cancer - diagnostic and therapeutic applications}

\section{Celso Reis ${ }^{1}$}

${ }^{1}$ University of Porto, Institute of Molecular Pathology and Immunology, Porto, Portugal

1. i3S - Instituto de Investigação e Inovação em Saúde, Universidade do Porto, Portugal

2. Ipatimup - Institute of Molecular Pathology and Immunology of the University of Porto, Portugal

3. ICBAS - Institute of Biomedical Sciences Abel Salazar of the University of Porto, Portugal

4. FMUP - Faculty of Medicine of the University of Porto, Portugal

Alterations of glycosylation are common on the cell surface during the carcinogenesis process and are associated with cancer progression and poor prognosis of the patients [1]. Gastric cancer is a heterogeneous disease that requires multidisciplinary treatment. Current targeted therapy depend on patient stratification based on molecular features of the tumor [2]. This study reports the glycomic and glycoproteomic analyses in human gastric cancer cells. Our results show that alterations of glycosylation impact the activation of tyrosine kinase receptors, such as MET (HGFR, Hepatocyte growth factor receptor) and RON (MSPR, Macrophage-stimulating protein receptor), and human epidermal growth factor receptor 2 (ErbB2) in gastric cancer cells $[3,4]$. We also report on the expression of truncated simple glycan antigens, as associated with molecular subtypes of tumors, such as those displaying microsatellite instability [5]. These results supports novel functional aspects of glycosylation modifications occurring in key proteins in cancer and their potential as cancer biomarkers for patient stratification and therapeutic intervention [6].

[1] Pinho SS, Reis CA. Nature Rev. Cancer 2015, 15, 540-555.

[2] Cristescu, R. et al. Nat. Med. 2015, 21, 449-456.

[3] Mereiter S, et al. Biochim. Biophys. Acta 2016, 1860, 1795-1808.

[4] Duarte, HO. et al. Int J Mol Sci. 2017, 18(11).

[5] Mereiter S, et al. J Clin Med. 2018 Sep 5;7(9).

[6] Mereiter S, et al. Cancer Cell. 2019 Jul 8;36(1):6-16. 


\section{POCT-Diagnostik: Trend und Zukunftsstrategien}

\section{V-11}

\section{Abgeleitete Diabetes-Parameter beim Einsatz von kontinuierlichen Glucose-Messverfahren}

\section{Guido Freckmann ${ }^{1}$}

${ }^{1}$ Institut für Diabetes-Technologie, Ulm, Germany

Immer mehr Menschen mit Typ 1 Diabetes nutzen kontinuierliche Glukosemonitoring Systeme (CGMSysteme) für ihre Diabetestherapie. Mithilfe der CGM-Systeme und der dazugehörigen Software können heutzutage verschiedene Daten und Parameter zur Beurteilung von Glukoseverläufen berechnet werden. CGM-Systeme ermitteln die Glukosekonzentrationen im interstitiellen Gewebe. Bei den sogenannten real-time CGM-Systemen (rtCGM) werden die Glukosekonzentrationen kontinuierlich erfasst (Messungen im ca. 5 Min. Takt). Während bei den intermittent scanning CGM Systemen (iscCGM) die Werte durch einen Scanvorgang angezeigt werden können. Die Tragezeit der CGM-Systeme beträgt in der Regel 6-14 Tage. Aus dieser Zeitspanne stehen Mess- sowie AnalyseDaten zur Verfügung, die mit verschiedenen Softwarepaketen eingesehen werden können. Insgesamt wird ein umfassender Einblick in den Glukoseverlauf der Anwender ermöglicht. Dabei ist zu beachten, dass die Hersteller unterschiedliche Software mit individuellen Einstellmöglichkeiten verwenden.

Ein internationales Konsensus Papier beschreibt und bewertet die Gesamtheit der aus CGM-Daten berechneten Parameter in Bezug auf deren Aussagekraft und Vergleichbarkeit mit dem zur Therapiekontrolle etablierten $\mathrm{HbA} 1 \mathrm{c}-$ Wert.

Beispiele für solche Parameter sind: Zeit im Zielbereich (Time in range; TiR), Zeit über/unter dem Zielbereich (Time above range; TaR and Time below range; TbR), Standardabweichung (SD), glykämische Variabilität (\%CV), der Glukosemanagement-Indikator ( $\mathrm{GMI}$ ) oder grafische Darstellungen wie das Ambulante Glukoseprofil (AGP).

Bei der Interpretation der Parameter sollte beachtet werden, dass im Gegensatz zu laboratoriumsmedizinischen Untersuchungen (Blutzucker und $\mathrm{HbA1c}$ ) für CGM-Systeme keine Qualitätsvorgaben und -kontrollen etabliert sind. Die Messwerte hängen vom CGM-System, den verwendeten Algorithmen und vom für die Kalibration genutzten Blutzuckermessgerät ab. Um Unterschiede zu minimieren ist es notwendig auch für CGM-Systeme eine Standardisierung vorzunehmen und Referenzen und Auswertungsprozeduren zu definieren.

Die aus den CGM-Daten gewonnenen Parameter können sehr hilfreiche Informationen über die aktuelle Stoffwechsellage des Patienten geben. So kann die Therapie überwacht und optimiert werden. Grafischen Darstellungen wie das AGP bieten die Möglichkeit einer schnellen Erfassung von Auffälligkeiten.

Derzeit können die Informationen aus CGM-Systemen als wertvolle Ergänzung zu dem HbA1c -Wert verwendet werden, nicht jedoch als Ersatz. 


\section{AG Leitlinien}

\section{V-12}

\section{S2k Leitlinie Präanalytik, Labor}

\section{Matthias Orth ${ }^{1}$; Johannes Peter Aufenanger ${ }^{2}$}

${ }^{1}$ Marienhospital Stuttgart, Institut für Laboratoriumsmedizin, Stuttgart, Germany ${ }^{2}$ Klinikum Ingolstadt, Institut für Laboratoriumsmedizin, Ingolstadt, Germany

Zur Sicherstellung von Patientensicherheit und um den größtmöglichen Nutzen von labormedizinischen Untersuchungen zu bekommen, ist neben Analytik und Befundinterpretation auch eine optimale Präanalytik notwendig. Hierzu zählt die Auswahl der am besten geeigneten Untersuchungen, eine korrekte Patientenvorbereitung, eine korrekte Probennahme und die korrekte Lagerung der Probe bis zur Analytik. Ziel der konsensbasierten Leitlinie unter Federführung der DGKL ist es, für die häufigsten Untersuchungen Mindestvorgaben aufzustellen, die dann auf nationalem Level umgesetzt werden können.

Bei der Auswahl der Laboruntersuchungen wird auf die Anforderung von sinnvollen Testkombinationen bei bestimmten medizinischen Fragestellungen wie auch auf die Auswahl geeigneter Reflextests fokussiert.

Bei der Festlegung der zulässigen Lagerungsbedingungen ist ein besonderer Schwerpunkt die Berücksichtigung der RiliBÄK-Vorgaben und der aktuellen Ergebnisse zur biologischen Variabilität der einzelnen Parameter und nicht, wie in manchen älteren Tabellen, auf fixe zulässige prozentuale Abweichungen. Eine Herausforderung ist die Angabe von zulässigen Lagerungszeiten bei wechselnden Umgebungsbedingungen, wie sie gerade im Einsendelabor kaum zu vermeiden sind.

Interessenten an einer Mitarbeit an dieser 2k-Leitlinie werden gebeten, sich an die Sektion Labormanagement der DGKL zu wenden. 


\title{
Präzisionsmedizin in der Pneumologie am Beispiel Allergie und Asthma
}

\section{V-13}

Neue pathophysiologische Konzepte: Endotypen und Biomarker

\author{
Harald Renz ${ }^{1}$
}

${ }^{1}$ Philipps Universität Marburg, Institut für Laboratoriumsmedizin, Marburg, Germany

Asthma is recognized as a heterogenous disease. Heterogeneity occurs on several levels, including the underlying chronic inflammatory response. The best characterized inflammatory response pattern is termed type-2 inflammation. This refers to a dominance of T-helper (Th)-2-cells with respective downstream events on the molecular and cellular level. Th-2-cytokines are not only produced by CD4+ T-cells but also by specialized innate lymphocytes (ILCs) and other cell population. The cytokine pattern is characterized by increased levels of IL-4, IL-5 and IL-13. These cytokines regulate many downstream events including the biology of eosinophils, mass-cell activation, epithelial responses and the production of IgE by B cells. Based on this paradigm patients suffering from type- 2 inflammation should potentially benefit from targeted therapies aiming to block defined molecules in this regulatory network. Such strategies have been developed using biologicals (monoclonal antibodies) directed against one or several of these cytokines or cytokine receptors. However, the development of type-2 inflammation depends on the expression of transcription factors which are necessary to direct T-cell specialization and maintenance of already specialized T-cells. For type-2 inflammation it is very well established that the transcription factor GATA-3 is sufficient and essential for the development of type2 responses. GATA-3 is not only expressed in CD4 Th-2 cells, but also in ILC-2 cells, mast cells, eosinophils and epithelial cells. Therefore, GATA-3 plays an essential role in type-2 inflammation. However, in order to therapeutically target this transcription factor an intervention strategy is required which is able to block GATA-3 on the intracellular level, since GATA-3 is not secreted. We developed a highly specific antisense molecule for GATA-3 messenger $(\mathrm{m})$ RNA which blocks the translation of GATA-3 mRNA into GATA-3 protein. Our antisense molecule (SB010) has, in addition to its specific binding activity to the GATA-3 mRNA, an additional cleavage activity due to an intrinsic catalytic domain of SB010 which cuts GATA-3 messenger RNA in at least two fragments. Phase lla proof-ofconcept clinical trial was performed in mild asthmatic patients. Treatment was carried out for 28 days by daily inhalation of a single dose of $10 \mathrm{mg} \mathrm{SB010}$. Allergen provocations were performed before and after the treatment cause. A primary endpoint of this study, significant reduction of the late phase asthmatic response, was reached, and in addition, also significant effect on the early phase allergic response was measured. The degree of improvement could be furthermore Phase lla proof a concept clinical trials were performed in asthma, COPD and ulcerative colitis, each with successful outcome. This is an example of precision medicine in the field of chronic inflammatory diseases where endotyping leads to novel precise therapeutic strategies. 


\section{a.u.la.-Symposium „update gynäkologische Endokrinologie“}

\section{V-14}

\section{Kontrazeption bei Thrombophilie}

\section{Günther Kappert ${ }^{1}$}

${ }^{1}$ Gerinnungszentrum Rhein-Ruhr, Gerinnungszentrum Rhein-Ruhr, Duisburg, Germany

In Deutschland sind ungefähr 20 Millionen Frauen im gebärfähigen Alter. Ein Drittel von ihnen nimmt kombinierte orale Kontrazeptiva (KOK) ein. Das Grundrisiko für Thromboembolien (TE) bei Frauen in diesem Alter ist gering, steigt jedoch, wenn KOK angewendet werden. Frauen haben daher bis zum Alter von 35 Jahren ein höheres Risiko für TE als Männer, nach diesem Zeitpunkt gleicht sich das Risiko an.

Die Erhöhung des Thromboserisikos hängt vom gewählten Kontrazeptivum ab. Ältere KOK mit Norgestimat / Levonorgestrel haben ein geringeres Risiko als neuere KOK. Folglich sind diese älteren KOK in vielen Richtlinien die erste Wahl. Neuere Generationen von Verhütungsmitteln sollten nur verschrieben werden, wenn ein anderer sekundärer Grund als die Empfängnisverhütung vorliegt. Darüber hinaus ist KOK mit Cyproteronacetat nur bei Hirsutismus und Akne vulgaris in Deutschland indiziert, obwohl es eine zuverlässige empfängnisverhütende Wirkung hat. Das Risiko für neuere KOK, die Estradiol E2 und nicht Ethinylestradiol EE enthalten, ist aufgrund fehlender Daten nicht klar. Die Anwendung der hormonellen Empfängnisverhütung mit Gestagen bringt mit Ausnahme des Depots Medroxyprogesteronacetat keine signifikante Erhöhung des Risikos für TE mit sich. Entsprechende Leitlinien sind bei der AWMF in Vorbereitung. Notfallverhütung, die nur Levonorgestrel oder Ulipristalacetat enthält, führt nicht zu einem höheren Risiko für TE und kann bei Frauen mit Thrombophilie angewendet werden.

Daher sind orale Desogestrel- oder Levonorgestrel-Kontrazeptiva ohne E2 oder EE, Intrauterinpessare (IUP) und Etonogestrel-Stäbchen die Verhütungsmittel der Wahl bei Frauen mit Thrombophilie.

Das Screening auf Thrombophilie ist nicht bei jeder Frau mit Empfängnisverhütungswunsch indiziert. Dies sollte auf bestimmte Fälle beschränkt werden, z. B. auf Frauen mit einer positiven Vorgeschichte für TE oder die nahe Verwandte besitzen, die in jungen Jahren eine TE erlitten haben. 


\section{Microbiome Signatures - Update 2019}

\section{V-15}

\section{Mikrobiomdiagnostik: Aktuelle Möglichkeiten und Probleme}

\section{André Gessner ${ }^{1}$}

${ }^{1}$ Universitätsklinik Regensburg, Institut für Med. Mikrobiologie und Hygiene, Regensburg, Germany

Im Jahr 2007 begann im Rahmen des amerikanischen Human Microbiome Projects (HMP) und des europäischen MetaHIT-Projekts die intensive und systematische Erforschung des „Mikrobioms“ mittels „Next Generation Sequencing“. Seitdem sind über 65.000 Publikationen zur Thematik in hochrangigen wissenschaftlichen Zeitschriften erschienen. Heute wissen wir: Der menschliche Körper beherbergt auf allen äußeren und inneren Oberflächen komplexe mikrobielle Ökosysteme, auch in Bereichen, die früher als steril galten. Die meisten dieser Studien hatten den Einfluss der Darmbakterien, der sogenannten gastrointestinalen Mikrobiota, auf verschiedene Erkrankungen des Menschen zum Inhalt. Basierend auf Erkenntnissen, dass intestinale Mikroorganismen die Darmbarriere, den Stoffwechsel, das Immunsystem und die Neurobiologie des Wirts entscheidend beeinflussen, ist die gezielte Modulation des menschlichen Mikrobioms ein vielversprechender Ansatzpunkt in der Prävention und Therapie zahlreicher Erkrankungen. Die medizinische Untersuchung des menschlichen Mikrobioms ist eine sehr anspruchsvolle Analytik, die zukünftig eine evidenzbasierte prophylaktische oder therapeutische Optimierung des Mikrobioms von Patienten ermöglichen soll. Durch systematische Standardisierung der aufwändigen Sequenzierungsverfahren, Entwicklung neuer Möglichkeiten der Stabilisierung von Patientenproben vor Eingang in das Labor sowie ein inzwischen von unserem Labor patentiertes Verfahren zur Quantifizierung und Qualitätskontrolle der Mikrobiomuntersuchung war es 2017 möglich, erstmals in Europa die Akkreditierung durch Regierungsbehörden für die Mikrobiomuntersuchung als medizinische Diagnostik für Patienten zu erhalten. Das Regensburger Mikrobiomlabor ist seit 2014 Qualitätsringversuchszentrum für Deutschland und andere europäische Länder. Die bisher durchgeführten fünf internationalen Ringversuche zeigten zwar hohe Reproduzierbarkeit der Mikrobiomanalytik in den teilnehmenden Laboratorien aber eine deutlich zu hohe Divergenz der Ergebnisse der verschiedenen Teilnehmer. Vor dem Einsatz der Mikrobiomanalytik zur Routinediagnostik und Therapiesteuerung individueller Patienten ist deshalb eine Standardisierung und Qualitätskontrolle der Untersuchungsverfahren dringend erforderlich. 


\section{V-16}

Thermodynamisch inspirierte Biomarker: Helfen uns Konzepte von Wettervorhersagen und der Klimamodellierung in der Medizin?

Rudi Balling ${ }^{1}$

${ }^{1}$ Universität Luxemburg, Luxembourg Centre for Systems Biomedicine, BELVAL, Luxembourg

Ich versuche die Teilnehmer davon zu überzeugen, dass Methoden aus der Physik, KlimaModellierung oder den Ingenieurs-Wissenschaften einen wichtigen Beitrag in der biologischen und medizinischen Forschung leisten können. Dazu werde ich auf die Frage ob man "Critical Transitions" mit Hilfe von thermodynamisch-basierten Methoden im Bereich der Pathogenese von Erkrankungen vorhersagen kann (Early Warning Signals). Weiterhin werde ich einen Vorschlag machen zu der Frage, ob verschiedenen chronischen Erkrankungen gemeinsame Mechanismen in der Pathogenese unterliegen (Common Mechanisms at Play). 


\title{
Rili-BÄK 2019
}

\section{V-17}

\section{Erweiterung der Rili-BÄK Tabelle B1 mit Messgrößen ohne Ringversuchspflicht}

\author{
Karl-Heinz Pick ${ }^{1}$ \\ ${ }^{1}$ VDGH, Berlin, Germany
}

Die Richtlinie der Bundesärztekammer zur Qualitätssicherung von laboratoriumsmedizinischen Untersuchungen (Rili-BÄK) regelt in ihrer aktuellen Fassung von 2014 die Grundlagen und Vorgehensweisen der Qualitätssicherung bei der Durchführung von laboratoriumsmedizinischen Untersuchungen, wobei das Regelwerk Mindeststandards vorgibt. Die Rili-BÄK unterliegt einem Prozess der kontinuierlichen Weiterentwicklung und Anpassung der vorgegebenen Qualitätsprozesse und Standards, um die Rili-BÄK an die Entwicklungen im Bereich der Durchführung von laboratoriumsmedizinischen Untersuchungen zu adaptieren bzw. zu gestalten.

Speziell für den Teil B1 - quantitative laboratoriumsmedizinische Untersuchungen ist es notwendig, die nach der letzten Revision der Rili-BÄK in 2014 festgelegten Messgrößen der Tabelle B1 kritisch zu betrachten und gegebenenfalls zu erweitern. Als Ansatz für die Erweiterung der Messgrößenliste wird das Modell der laborinternen Fehlergrenzen für die Festlegung von Fehlergrenzen verwendet. Weiterhin gilt für die neu aufzunehmenden Messgrößen vorerst keine Ringversuchspflicht.

Die Rili-BÄK Arbeitsgruppe für die Überarbeitung des speziellen Teils B1 hat mittels einer Umfrage bei den zuständigen Fachgesellschaften und anderen betroffenen Kreisen zuerst diejenigen Messgrößen identifiziert, die für eine Aufnahme in eine Tabelle (Tabelle B1 a - Messgrößen ohne Ringversuchspflicht) geeignet sind. Auswahlkriterium waren Häufigkeit und medizinischer Nutzen jeder Messgröße. Aus den Rückmeldungen wurden 30 Messgrößen ausgewählt, für die in einer Laborbefragung durch die Referenzinstitute RfB und INSTAND die Qualitätsparameter Unpräzision, Unrichtigkeit und QMMA abgefragt wurden. Mit Hilfe dieser Daten und unter Berücksichtigung der in der Rili-BÄK bereits etablierten Formel zur Berechnung von Laborinternen Fehlergrenzen setzte die Arbeitsgruppe die Mindestanforderungen an das erlaubte Fehlerintervall und den jeweiligen Gültigkeitsbereich für die Anwendung dieses Intervalls fest. Auch hier gilt, wenn durch den Hersteller eines Tests für diesen engere Fehlergrenzen angegeben sind, müssen diese verwendet werden.

Die neue Tabelle B1 a - mit Messgrößen ohne Ringversuchsverpflichtung - setzt einen ersten Startpunkt zur Erweiterung der Messgrößen mit vorgegebenen Fehlergrenzen. Ziel zukünftiger RiliBÄK Revisionen sollte darin bestehen, die Anforderung an die Messqualität anhand gut definierter medizinischer Erfordernisse weiter zu entwickeln, sei es durch entsprechende Vorgaben für Ringversuche oder auch durch Verengung der erlaubten Fehlergrenzen. 


\section{Data mining im Labor}

\section{V-18}

\section{Applying machine learning to bio-specimen - how epigenetic modification can reveal tissue sample classification}

\section{Alexander Tolios ${ }^{1}$}

${ }^{1}$ Medizinische Universität Wien, Universitätsklinik für Blutgruppenserologie und Transfusionsmedizin, Wien, Austria

Introduction: The major impact of DNA methylation on protein biosynthesis in tissues is well-known. Those epigenetic changes should also be usable to differentiate between different tissue types. We evaluated if machine learning techniques can be used to differentiated between different origin tissue. Methods: Epigenetic DNA information from The Cancer Genome Atlas (TCGA) was used for all subsequent analyses. DNA methylation calculated as beta-values of approx. 650 samples was used for all analysis. Data was separated into two different datasets and used either for training or algorithm testing. PCA (principal component analysis) was used to reduce data dimensionality. Sample classification was performed using both linear and non-linear algorithms with different numbers of principal components. Model performance was enhanced using nested crossvalidation.

Results: Up to 6 principal components led to an increase in predictive performance. Those results were consistent throughout all algorithms. A higher number of PCs did not yield a higher accuracy. The following algorithms were used: penalized logistic regression, linear discriminant analysis, knearest neighbors, support vector machines, algorithms neural networks and random forests. Most algorithms produced accurate results (accuracy $>90 \%$ ). More complex algorithms generally outperformed simpler ones on the training dataset. Classification results (on the training data) were the highest when using a random forest (accuracy > 95\%, Cl 92-98\%). The results on the independent test dataset were comparable (accuracy $>95 \%$, Cohen's Kappa $>0.90$ ).

Conclusion: In our study we could show that it was possible to accurately differentiation between different tissue samples using machine learning algorithms. A technology like this might be useful in cases where classical histopathological analysis is not possible or error prone. 